\title{
Simulação perfeita de cadeias de alcance variável não limitado
}

\author{
Alexsandro Giacomo Grimbert Gallo
}

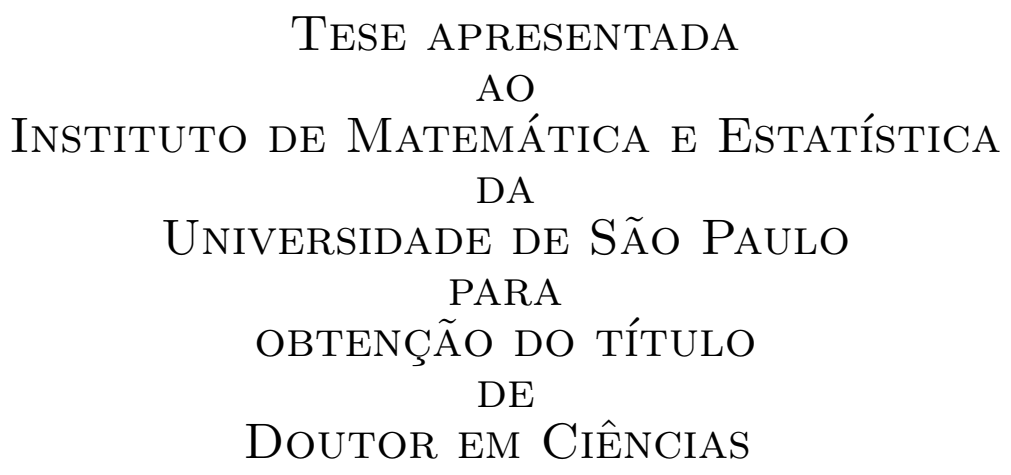

Programa: Estatística

Orientador: Prof. Dr. Antonio Galves

Durante o desenvolvimento deste trabalho o autor recebeu auxílio financeiro da CAPES, CNPq e FAPESP

São Paulo, Novembro de 2009 


\section{Simulação perfeita de cadeias de alcance variável não limitado}

Este exemplar corresponde à redação

final da tese devidamente corrigida

e defendida por Alexsandro Gallo

e aprovada pela Comissão Julgadora.

Banca Examinadora:

- Prof. Antonio Galves (orientador) - IME-USP.

- Profa. Dra. Florencia Graciela Leonardi - IME-USP.

- Profa. Dra. Nancy Lopes Garcia - UNICAMP.

- Prof. Dr. Jorma Johannes Rissanen - UT.

- Prof. Dr. Aurélien Garivier - ENST. 


\section{Agradecimentos}

Ao Brasil por tudo.

Ao Prof. Antonio Galves, que me orientou durante esta tese. Ele me fez descobrir o mundo da pesquisa com muita dedicação e muito entusiasmo.

Agradeço a todo mundo no IME, amigos, funcionários e professores por fazer desta instituição um lugar agradável e muito empolgante para a pesquisa.

Obrigado ao NUMEC, aos seus funcionários (sobre tudo Patty) e aos seus utilizadores (os estudantes

e pesquisadores que eu não estou citando). É um lugar realmente muito bom para estudar. Este ambiente foi particularmente bom para mim, sobre tudo graça à disponibilidade e simpatia de Christophe e Daniel com quem convivi muito neste último ano.

O compromisso oficioso das terça feira e sexta feira foi capital para meu desenvolvimento intelectual, agradeço portanto a todos os participantes.

Agradeço a minha família, que me segue e me dá todo o apoio que preciso, de longe (papa, maman, mamé...)

Enfim, agradeço à Nati, minha companheira e namorada (o raio de sol de uma manhã de verão quando choveu no dia anterior e que....). 


\section{Resumo}

Nesta tese consideramos cadeias de alcance variável não limitado. São cadeias de alcance infinito cuja família de probabilidades de transição é representada por uma árvore de contextos probabilística. Dado uma árvore de contextos probabilística não limitada, as questões que nos interessam são as seguintes: existe ou não uma cadeia estacionária compatível com esta árvore? Se existir, esta cadeia é única? Podemos fazer uma simulação perfeita desta cadeia?

Nesta tese, apresentamos novos critérios suficientes que garantem a existência e a unicidade da cadeia estacionária e, sob restrições mais fortes, a possibilidade de fazer uma simulação perfeita.

Uma caraterística interessante do nosso trabalho é o fato de não utilizarmos a condição de continuidade.

Palavras-chave: Árvores de contextos probabilística, Cadeias de alcance variável, Simulação perfeita, Esquema regenerativo. 


\section{Abstract}

We present a new perfect simulation algorithm for stationary chains (indexed by $\mathbb{Z}$ ) having unbounded variable length memory. This is the class of infinite memory chains for which the family of transition probabilities is given by probabilistic context tree. Our condition is expressed in terms of the structure of the context tree. In particular, we do not assume the continuity of the family of transition probabilities. We give an explicit construction of the chain using a sequence of i.i.d. random variables uniformly distributed in $[0,1[$.

Keywords: Probabilistic context tree, Variable length memory chains, Perfect simulation, Regeneration scheme. 


\section{Sumário}

Lista de Figuras $\quad$ ix

1 Introdução 1

1.1 Motivação . . . . . . . . . . . . . . . . . . . . . . . . 1

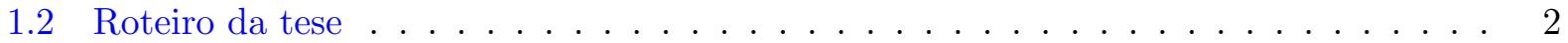

2 Preliminares $\quad 5$

2.1 Notação . . . . . . . . . . . . . . . . . . . . . . . . . . . 5

2.2 Sobre cadeias de alcance infinito f . . . . . . . . . . . . . . . 6

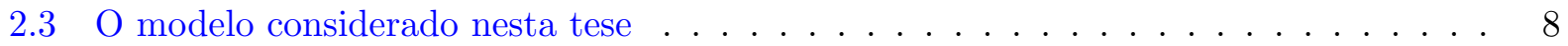

2.3.1 Árvores de contextos probabilísticas . . . . . . . . . . . . . . 8

2.3.2 Cadeias de alcance variável não limitado . . . . . . . . . . . . . . . . . 10

2.4 Construção de uma c.a.v. dado um passado fixado . . . . . . . . . . . . . . . 11

2.5 A simulação perfeita e suas consequências . . . . . . . . . . . . . . . 13

2.5.1 Introdução informal . . . . . . . . . . . . . . . . . . . . . . 14

2.5.2 O Algoritmo . . . . . . . . . . . . . . . . . 15

2.5.3 Um exemplo de simulação perfeita . . . . . . . . . . . . . . . . . 17

2.5.4 Consequências: existência e unicidade . . . . . . . . . . . . . . . . . 19

2.6 Esquema regenerativo . . . . . . . . . . . . . . . . . . . . . 24

2.7 Aproximação Markoviana . . . . . . . . . . . . . . . . . . . 26

3 Árvores não limitadas: resultados introdutórios $\quad 29$

3.1 Um exemplo de não existência . . . . . . . . . . . . . . . . . . . . . . . 29

3.2 Probabilidade positiva de ter um contexto de tamanho infinito . . . . . . . . . 30

3.3 Exemplo em que a cadeia não é irredutível . . . . . . . . . . . . . . . . . . 31

3.4 Probabilidade zero de ter um contexto de tamanho infinito . . . . . . . . . . . 31

4 Árvores tendo um contexto terminal 35

4.1 Existência e unicidade da medida invariante . . . . . . . . . . . . . . . 37

4.2 Cálculo explícito da medida invariante . . . . . . . . . . . . . . . . . 40

4.3 Reversibilidade da cadeia esparsa . . . . . . . . . . . . . . . . . . . . . . . . . . . . . . . . . . . . . . . .

4.4 Entropia da cadeia esparsa . . . . . . . . . . . . . . . . . . . 43 
4.5 Simulação perfeita . . . . . . . . . . . . . . . . . . . 45

5 Extensão para árvores gerais: resultados principais da tese 49

5.1 Partição das árvores e bons contextos . . . . . . . . . . . . . . . . . . 49

5.2 Exemplos de árvores com bons contextos . . . . . . . . . . . . . . . . 50

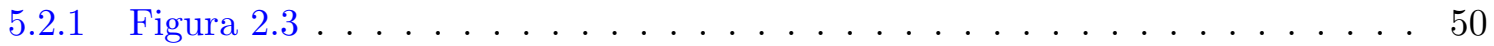

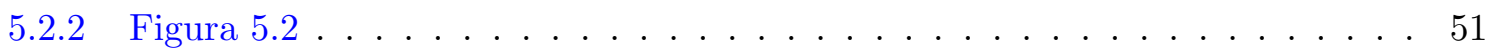

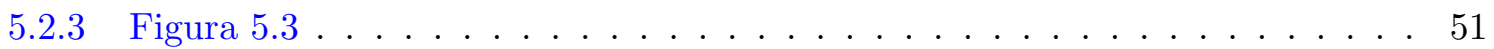

5.3 Enunciado dos resultados . . . . . . . . . . . . . . . . 52

6 Prova do Teorema $5.2 \quad 55$

6.1 Prova do Lema $6.1 \ldots \ldots \ldots$. . . . . . . . . . . . . . . . . . 57

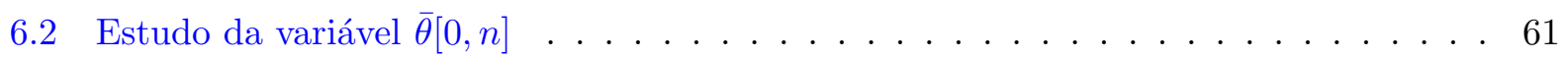

6.3 Prova do teorema . . . . . . . . . . . . . . . . . 71

$\begin{array}{lll}7 & \text { Prova do Teorema } 5.3 & 75\end{array}$

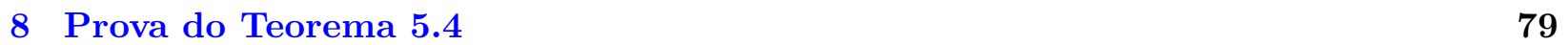

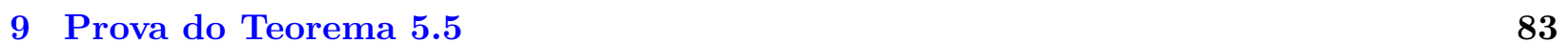

10 Conclusion $\quad 95$

$\begin{array}{lr}\text { Referências Bibliográficas } & 97\end{array}$ 


\section{Lista de Figuras}

2.1 Exemplos de árvores de contextos probabilísticas . . . . . . . . . . . . . 10

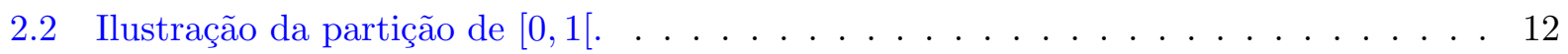

2.3 Árvore sobre $A=\{0,1\}$ tendo 1 como bom contexto . . . . . . . . . . . 19

2.4 Exemplo de simulação perfeita da cadeia compatível com a árvore da Figura 2.3. . 20

2.5 Árvore da Figura 2.3 truncada na altura $4 . \ldots \ldots$. . . . . . . . . . . 27

2.6 Árvore da Figura 5.2 truncada na altura 5. Os pequenos triângulos representam os triângulos da árvore inicial truncados na altura indicada. . . . . . . . . . . . 27

4.1 Árvore sobre $A=\{0,1\}$ tendo 01 como contexto terminal. . . . . . . . . . . 35

4.2 Árvore sobre $A=\{0,1,2\}$ tendo o símbolo 1 como contexto terminal. . . . . . . 36

5.1 Árvore sobre $A=\{0,1\}$ tendo 1 como bom contexto . . . . . . . . . . 51

5.2 Árvore sobre $A=\{0,1\}$ tendo 01 como bom contexto . . . . . . . . . . . 52

5.3 Árvore sobre $A=\{0,1,2\}$ tendo 1 como bom contexto . . . . . . . . . . . 52

6.1 Ilustração da variável $\bar{\theta}[0, n]$ construída utilizando $\overline{\mathbf{Z}}$. . . . . . . . . . . . . . . 62

6.2 As cadeias $\mathbf{C}^{(n)}, \mathbf{C}^{\left(n^{\prime}\right)}, \mathbf{D}^{(n)}$ e $\mathbf{D}^{\left(n^{\prime}\right)}$ construídas utilizando $\overline{\mathbf{Z}}$ e os valores (6.16) para $\bar{r} .67$

6.3 Ilustração da equação (6.21). A sequência de "??" significa que pode ocorrer qualquer coisa nestes tempos, desde que a cadeia $\mathbf{D}^{(0)}$ não toca $0 \ldots \ldots \ldots \ldots$ 


\section{Capítulo 1}

\section{Introdução}

\subsection{Motivação}

Cadeias de alcance variável são uma classe de cadeias estocásticas que foram introduzidas no artigo de Jorma Rissanen intitulado A universal data compression system (1983) como um modelo para compressão de dados em teoria da informação. Estas cadeias têm a seguinte propriedade. Para escolher o próximo símbolo da cadeia, precisamos conhecer um sufixo do passado (o contexto), cujo tamanho (limitado no caso em que Rissanen introduziu o modelo) depende dos valores assumidos pela cadeia neste passado. Uma consequência dessa definição informal é que as probabilidades de transição podem ser representadas sob a forma de uma árvore de contextos probabilística $(\tau, p)$. Esta árvore de contextos probabilística tem o mesmo papel que a matriz de transição para as cadeias de Markov, e ela contém toda a informação de dependência no passado da cadeia. Este modelo foi desde então aplicado com sucesso para modelagem de dados em diversas áreas tais como teoria da informação (Rissanen (1983)), biologia (Bejerano \& Yona (2001), Leonardi (2006)) ou ainda lingüística (Galves et al. (2009)). Esta aplicabilidade é devida em parte ao fato que a árvore de contextos recupera as dependências estruturais dos dados estudados. Referimos à tese de doutorado de Leonardi (2007) sobre este assunto.

Nesta tese, estudamos cadeias de alcance variável e não limitado. O fato que a memória tenha alcance não limitado significa que se trata de um classe de cadeias de alcance infinito. A literatura das cadeias de alcance infinito começou com os artigos pioneiros de Onisescu nos anos 30 para

modelar um problema de urnas. É uma generalização natural das cadeias de Markov no caso em que as probabilidades de transição dependem de todo o passado da cadeia. Uma motivação para 
se considerar tais cadeias é que elas modelam de maneira mais realista os sistemas unidimensionais da física estatística do que as cadeias de Markov (ou seja o modelo de Ising). Portanto há uma motivação clara em física estatística (generalisações do modelo de Ising unidimensional), e em particular, há uma ligação importante entre cadeias de alcance infinito, estados de Gibbs e sistemas dinâmicos apresentando regularidades como difeormorfismos de Anosov e Axioma A (ver por exemplo Bowen (2008)).

Devido à sua relação com sistemas dinâmicos e estados de Gibbs, a literatura das cadeias de alcance infinito utiliza sistematicamente a hipótese de continuidade. Na presente tese, propomos uma nova abordagem utilizando as árvores de contexto, e sem nunhuma necessidade de assumir a condição de continuidade. O estudo das propriedades das cadeias de alcance variável não limitado parece ser novo. Um primeiro passo nessa direção foi feito na tese de doutorado de Esteves (2007). Lá foi demonstrada a existência de um esquema regenerativo para cadeias binárias, contínuas e usando como contexto de referência para o esquema o símbolo 1. Nós generalizamos este trabalho em várias direções: alfabeto qualquer, contexto de referência qualquer, e o mais importante, não assumimos a continuidade. Isso é possível devido a uma outra novidade desta tese: a utilização do esquema regenerativo como base para simulação perfeita da cadeia.

\subsection{Roteiro da tese}

Esta tese está organizada da seguinte maneira.

- No primeiro capítulo, introduzimos as definições assim como a notação necessária para introduzir formalmente as questões de interesse na teoria das cadeias de alcance infinito. Poderemos então definir o modelo das cadeias de alcance variável e depois explicar a abordagem construtiva inspirada em Fernández et al. (2001) e utilizada nesta tese.

Com tudo isso em mãos, poderemos explicar como funciona o algoritmo de simulação perfeita para as cadeias de alcance variável não limitado.

Provamos então que quando este algoritmo é factível (o algoritmo termina quase-certamente num tempo finito), a amostra obtida na saída é uma amostra da única cadeia estacionária compatível com a nossa família de probabilidades de transição.

Também introduzimos a noção de esquema regenerativo, e mostramos que sob certas condições, 
a simulação perfeita implica a existência de um esquema regenerativo.

- O capítulo 2 tem dois objetivos. Primeiro, apresentar alguns exemplos simples exibindo comportamentos patológicos, e segundo, achar alguns critérios simples para garantir que tais comportamentos não aconteçam.

- No capítulo 3 estudamos as árvores de contextos tendo um contexto terminal. Muitas contas explícitas podem ser feitas neste caso simples, porque correspondem às chamadas cadeias de renovação. O objetivo deste capítulo é introduzir alguns resultados que utilizamos depois na tese. Em particular, provamos que se o contexto terminal tem uma probabilidade estritamente positiva de ocorrer (uniformemente sobre todos os passados), então o algoritmo de simulação perfeita descrito no capítulo 2 é factível. A prova neste caso simples motiva a prova para casos mais gerais.

- No quarto capítulo, introduzimos uma classe de árvores mais geral e enunciamos os resultados da tese.

- Os últimos capítulos estão dedicados às provas dos resultados. 


\section{Capítulo 2}

\section{Preliminares}

\subsection{Notação}

Seja $A$ um alfabeto enumerável de cardinal \#A (possivelmente infinito: $2 \leq \# A \leq+\infty$ ). Em geral, este alfabeto será denotado por $\{1,2, \ldots, \# A\}$. Nos exemplos, que são limitados aos alfabetos de 2 ou 3 letras, usaremos os alfabetos $\{0,1\}$ ou $\{0,1,2\}$ por convenção. Denotamos por

$$
A^{-\mathbb{N}}=A^{\{\ldots,-2,-1\}}
$$

o conjunto das sequências semi-infinitas a esquerda, por

$$
A^{\mathbb{Z}}=A^{\{\ldots,-1,0,1, \ldots\}}
$$

o conjunto das sequências bi-infinitas de símbolos, e por

$$
A^{*}=\bigcup_{j=0}^{+\infty} A^{\{-j, \ldots,-1\}}
$$

o conjunto das sequências finitas de símbolos. Nesta última notação, o caso $j=0$ corresponde à sequência vazia $\emptyset$. Também, para todo inteiro $k,-\infty<k<+\infty, A^{k}$ denota o conjunto das sequências de $k$ símbolos. Dados dois inteiros $-\infty<m \leq n<+\infty$, denotamos por $a_{m}^{n}$ a sequência $\left(a_{m}, \ldots, a_{n}\right)$ de símbolos em $A$, e por $\left|a_{m}^{n}\right|=n-m+1$ seu comprimento. Uma convenção útil para algumas definições é $a_{n+1}^{n}:=\emptyset$. Também, escrevemos $a_{-\infty}^{n}$ a sequência $\left(\ldots, a_{n-1}, a_{n}\right)$ semi-infinita a esquerda de elementos de $A$ e a a sequência $\left(\ldots, a_{-1}, a_{0}, a_{1}, \ldots\right)$ bi-infinita de elementos de $A$. 
Enfim, escrevemos para todo $i, a_{m+i}^{n+i} \in A^{n-m+1} \subset A^{\star}$ e $a_{-\infty}^{i} \in A^{-\mathbb{N}}$.

Dadas duas sequências $w$ e $v$ de $A^{\star}$, denotaremos por $v w$ a sequência de tamanho $|v|+|w|$ obtida concatenando $v$ e $w$. A concatenação de sequências é estendida ao caso em que $v$ é um elemento de $A^{-\mathbb{N}}$. Se $n$ é um inteiro não nulo e $v$ um elemento de $A^{\star}$, denotamos por $v^{n}$ a concatenação de $n$ vezes a sequência $v$. Enfim, se $v \in A^{\star}$ e $w \in A^{\star} \cup A^{-\mathbb{N}}$ são tais que $|v| \leq|w|$, escrevemos (por abuso de notação) $v \in w$ ou $v \notin w$ para dizer que $v$ é ou não é uma subsequência de $w$.

\subsection{Sobre cadeias de alcance infinito}

Nesta parte, introduzimos rapidamente as definições e os resultados da literatura sobre cadeias de alcance infinito. Como já mencionamos na introdução, nós adotamos nesta tese a abordagem construtiva introduzida no livro de Fernández et al. (2001). Em particular, as questões de mensurabilidade se referem sempre à $\sigma$-álgebra natural, e são sempre imediatas, sendo que todas as funções introduzidas nesta tese são claramente mensuráveis. Portanto, da mesma maneira que foi feito no livro de Fernández et al. (2001), definimos para todo o resto da tese que a $\sigma$-álgebra

- de $[0,1]$ é a $\sigma$-álgebra de Lebesgue,

- de um produto finito ou infinito de $A$ é o produto da $\sigma$-álgebra discreta sobre $A$,

- de um produto destes espaços é a $\sigma$-álgebra produto correspondente.

Definição 2.1 (Família de probabilidades de transição). Uma função $P: A \times A^{-\mathbb{N}} \rightarrow[0,1]$ tal que

- para todo $a \in A$, a função $P(a \mid \cdot)$ é mensurável em relação à $\sigma$-álgebra produto,

- e para todo $a_{-\infty}^{-1} \in A^{-\mathbb{N}}$

$$
\sum_{a \in A} P\left(a \mid a_{-\infty}^{-1}\right)=1
$$

é chamada de família de probabilidades de transição.

É também chamado de núcleo de probabilidade, de especificação em física estatística, ou de $g$-função em teoria ergódica. Nesta definição podemos ver que $P$ não depende do tempo, isto significa que a família é homogênea. É o caso de todas as famílias de probabilidades de transição estudadas nesta tese. Uma cadeia estocástica sobre o alfabeto $A$ é uma sequência bi-infinita de variáveis aleatórias (a valor em $A$ ): $\left(X_{n}\right)_{n \in \mathbb{Z}}, X_{i} \in A$. Adotamos a notação $\mathbf{X}:=\left(X_{n}\right)_{n \in \mathbb{Z}}$. 
Definição 2.2 (Cadeia compatível com uma família de probabilidades de transição). Uma cadeia $\mathbf{X}$ de lei $\mathbb{P}$ é compativel com $P$ se para $\mathbb{P}$-quase-todo $a_{-\infty}^{-1} \in A^{-\mathbb{N}}$ e todo a $\in A$ temos

$$
\mathbb{P}\left(X_{0}=a \mid X_{-\infty}^{-1}=a_{-\infty}^{-1}\right)=P\left(a \mid a_{-\infty}^{-1}\right)
$$

Estas cadeias são

- cadeias de Bernoulli se $P\left(a \mid a_{-\infty}^{-1}\right)$ não depende de $a_{-\infty}^{-1}$,

- cadeias de Markov de ordem $K$ se $P\left(a \mid a_{-\infty}^{-1}\right)=P\left(a \mid b_{-\infty}^{-1}\right)$ sempre que $a_{-K}^{-1}=b_{-K}^{-1}$, para algum $K$ finito,

- de alcance infinito caso contrário. Neste caso dizemos que a família de probabilidades de transição é não limitada.

O caso que nos interessa é o das cadeias de alcance infinito. A Definição 2.2 é equivalente a dizer que a cadeia $\mathbf{X}$ é compatível com $P$ se esta última é uma versão regular das probabilidades condicionais da primeira. Dado uma família de probabilidades de transição não limitada, a existência de uma cadeia estacionária compatível é uma questão não-trivial e precisamos impor condições sobre $P$. Na nossa terminologia, é equivalente dizer que existe uma cadeia estacionária compatível com $P$ ou dizer que existe uma lei invariante compatível com $P$. Uma quantidade muito utilizada na literatura destas cadeias é a taxa de continuidade da família de probabilidades de transição:

Definição 2.3 (Taxa de continuidade). Dado uma familia de probabilidades de transição P, a taxa de continuidade é definida por

$$
\beta_{k}=\sup \left\{\left|P\left(a \mid a_{-\infty}^{-1}\right)-P\left(a \mid b_{-\infty}^{-1}\right)\right|: a \in A, a_{-\infty}^{-1} \stackrel{k}{=} b_{-\infty}^{-1}\right\}
$$

onde $a_{-\infty}^{-1} \stackrel{k}{=} b_{-\infty}^{-1}$ significa $a_{-k}^{-1}=b_{-k}^{-1}$

Dizemos que $P$ é contínua se $\beta_{k}$ converge para zero quando $k$ diverge. Assumir que a família de probabilidades de transição seja contínua é uma maneira de assumir que as probabilidades de transição não dependem muito do passado remoto. Sabe-se que existe ao menos uma cadeia estacionária compatível com uma família $P$ contínua (ver por exemplo Keane (1972)). Para saber 
se esta cadeia é única, existem critérios sobre a taxa de continuidade (a taxa na qual $\beta_{k}$ vai para 0). Sem entrarmos em detalhes, mencionamos que Doeblin \& Fortet (1937) foram os primeiros a considerar tais critérios, e apresentaram os primeiros resultados sobre a velocidade de convergência para a medida invariante. Em seguida, uma longa lista de autores que inclui Harris (1955), Berbee (1987), Lalley (1986), Fernández \& Galves (2002) estenderam esses resultados, mostrando diferentes caraterísticas da única cadeia estacionária (aproximação Markoviana, esquema regenerativo), sob condições mais fracas. No entanto, foi Stenflo (2003) que obteve o resultado com condição mais fraca utilizando esta noção de continuidade, mostrando que se a sequência $\left(\beta_{k}\right)_{k \geq 0}$ está em $\ell^{2}$ (quadrado somável) então há unicidade. É importante notar que nesse artigo, Stenflo (2003) considera o caso de um alfabeto finito, e cujos elementos do alfabeto aparecem ao longo da realização com uma probabilidade de transição uniformemente limitada inferiormente (fortemente não nulo) hipótese que não aparece em vários trabalhos da lista citada acima.

\subsection{O modelo considerado nesta tese}

\subsection{1 Árvores de contextos probabilísticas}

Dizemos que uma sequência $s$ é sufixo de uma outra sequência $v$ se $|s| \leq|v|$ e $v_{-|s|}^{-1}=s$.

Definição 2.4. Um subconjunto $\tau$ de $A^{*} \cup A^{-\mathbb{N}}$ é uma árvore se nenhum elemento $s \in \tau$ é um sufixo de um outro elemento $v \in \tau$. Essa propriedade é chamada propriedade do sufixo.

Se a sequência vazia $\emptyset$ pertence a $\tau$, segue da propriedade do sufixo que $\tau=\{\emptyset\}$, ou seja a árvore limitada à sua raíz.

Definição 2.5. Quando $\sup \{|v|: v \in \tau\}=+\infty$, dizemos que $\tau$ é ilimitado.

Definição 2.6. Uma árvore $\tau$ é completa se todo elemento $a_{-\infty}^{-1}$ de $A^{-\mathbb{N}}$ tem um sufixo pertencendo a $\tau$. A propriedade do sufixo implica que este sufixo é único. Chamamos este sufixo de contexto da sequência $a_{-\infty}^{-1}$ e ele é denotado por $c_{\tau}\left(a_{-\infty}^{-1}\right)$. Uma árvore completa é chamada árvore de contextos.

Estendemos também a noção de contexto para as sequências finitas: para todo $a_{m}^{n} \in A^{\star}, m \leq n$, escrevemos $c_{\tau}\left(a_{m}^{n}\right)=v$ se $v$ é um sufixo de $a_{m}^{n}$ pertencendo a $\tau$. Se não há contexto de $\tau$ que seja sufixo de $a_{m}^{n}$, usamos a convenção $c_{\tau}\left(a_{m}^{n}\right)=\emptyset$. Observamos em particular que com a notação $a_{n+1}^{n}=\emptyset$, obtemos sempre $c_{\tau}\left(a_{n+1}^{n}\right)=\emptyset$. 
Definição 2.7. Uma árvore probabilística sobre A é um par ordenado $(\tau, p)$ onde

1. $\tau$ é uma árvore de contextos,

2. $p=\{p(\cdot \mid v) ; v \in \tau\}$ é uma familia de probabilidades de transição sobre A.

Árvores probabilísticas não limitadas são simplesmente famílias de probabilidades de transição. Exemplo de árvores de contexto probabilísticas estão dados pelas Figuras 2.1(a) (no caso limitado) e 2.1(b) (no caso ilimitado). A primeira é definida sobre o alfabeto $\{1,2,3\}$

$$
\tau=\{2,3,11,21,131,231,331\}
$$

e os números escritos nas caixas abaixo de cada folha da árvore (contextos) são as probabilidades de transição para os símbolos 1, 2 e 3 dado estes contextos. A segunda árvore é definida sobre o alfabeto $A=\{0,1\}$ da seguinte maneira:

$$
\tau=\left\{0^{\infty}\right\} \cup\{1,10,100, \ldots\}=\left\{0^{\infty}\right\} \cup\left\{10^{i}\right\}_{i \geq 0},
$$

As probabilidades de transição estão denotadas por $p_{i}=p\left(1 \mid 0^{i} 1\right)=1-p\left(0 \mid 0^{i} 1\right)$.

Observaçoes 2.8. Chamamos a atenção sobre a notação para as probabilidades condicionais e as probabilidades de transição: escrevemos

$$
\mathbb{P}\left(X_{0}=a_{0} \mid X_{-1}=a_{-1}, X_{-2}=a_{-2}, \ldots\right),
$$

$e$

$$
p\left(a_{0} \mid a_{-1} a_{-2} \ldots\right)
$$

Isto é, dentro do condicionamento, invertemos o tempo, para ter uma continuidade temporal na notação: $0,-1,-2, \ldots$

Exemplos de árvoves de contextos não limitadas mais gerais (sem especificar as probabilidades de transição) são dados pelas Figuras 2.3 and 5.2. 


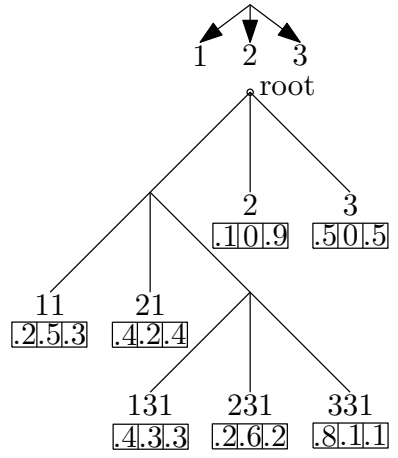

(a)

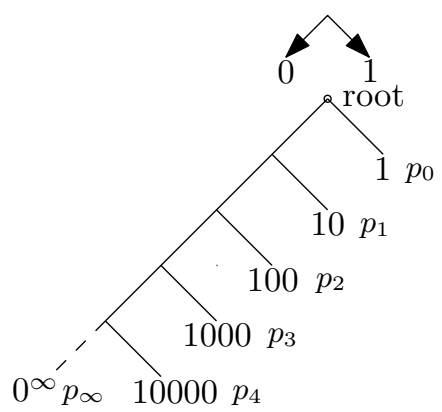

(b)

Figura 2.1: Exemplos de árvores de contextos probabilísticas

Definição 2.9. Dizemos que um elemento $a \in A$ é $\epsilon$-regular se

$$
\inf _{v \in \tau} p(a \mid v) \geq \epsilon>0
$$

Uma sequência $v$ de $A^{\star}$ também é dita $\epsilon$-regular se todos os símbolos de A que aparecem em $v$ são $\epsilon$-regulares.

\subsubsection{Cadeias de alcance variável não limitado}

Definição 2.10. Dizemos que uma cadeia estocástica e estacionária $\mathbf{X}$ de lei $\mathbb{P}$ é compatível com uma árvore probabilística $(\tau, p)$ se para $\mathbb{P}$-q.t. os passados $a_{-\infty}^{-1} \in A^{-\mathbb{N}}$ e todo a $\in$ A temos

$$
\mathbb{P}\left(X_{0}=a \mid X_{-\infty}^{-1}=a_{-\infty}^{-1}\right)=p\left(a \mid c_{\tau}\left(a_{-\infty}^{-1}\right)\right)
$$

Estas cadeias são chamadas cadeias de alcance variável. Se $\tau$ é ilimitado, a cadeia é chamada cadeia de alcance variável não limitado.

Estas definições são relacionadas com as definições da Seção 2.2 sobre as cadeias de alcance infinito genéricas. Como mencionado na seção anterior, o critério clássico na literatura das cadeias de alcance infinito para assegurar a existência de uma cadeia estacionária compatível com uma dada família de probabilidades de transição é exigir que esta família seja contínua. A vantagem de estudar as famílias de probabilidades de transição sob a forma de árvores probabilísticas vem do fato de podermos usar a informação sobre a estrutura dos passados (a árvore de contextos) para 
não ter que assumir a continuidade.

Esta tese é sobre cadeias de alcance variável não limitado e não discutiremos o caso limitado. Portanto, as vezes, não mencionaremos que estamos nos referindo ao caso não limitado. Usaremos as vezes a notação c.a.v. para cadeias de alcance variável (não limitado).

\subsection{Construção de uma c.a.v. dado um passado fixado}

Nesta seção, explicamos como funciona a abordagem construtiva das cadeias de alcance infinito. Consideramos o caso das c.a.v., embora este procedimento pode ser estendido para cadeias de alcance infinito genéricas. Referimos ao livro de Fernández et al. (2001) para uma exposição completa sobre cadeias de alcance infinito genéricas.

Seja U uma sequência de variáveis aleatórias i.i.d. uniformemente distribuídas no intervalo $[0,1[$, denotamos por $(\Omega, \mathcal{F}, \mathbb{P})$ o espaço de probabilidade associado. Também denotaremos por $\mathbb{E}$ a esperança em relação à medida $\mathbb{P}$. A maioria das cadeias consideradas nesta tese são contruídas utilizando $\mathbf{U}$.

Suponha que temos uma árvore probabilística $(\tau, p)$ sobre um alfabeto enumerável $A$. Denotamos por $\mathcal{E}$ o subconjunto (eventualmente vazio) de $A$ tendo todos os elementos $\epsilon$-regulares para algum $\epsilon>0$. Consideramos sem perda de generalidade $A=\{1, \ldots, \# A\}(\operatorname{com} \# A \leq+\infty)$ e $\mathcal{E}=\{1, \ldots, \# \mathcal{E}\}$, se $\mathcal{E} \neq \emptyset$. Para todo $v \in \tau$ definimos um conjunto de intervalos disjuntos formando uma partição de [0,1[ da seguinte maneira: para todo a em $\mathcal{E}$

$$
J(a \mid \emptyset)=\left[(a-1) \epsilon, a \epsilon\left[, \quad J(a \mid v)=\left[\# \mathcal{E} \epsilon+\sum_{i=1}^{a-1}(p(i \mid v)-\epsilon), \# \mathcal{E} \epsilon+\sum_{i=1}^{a}(p(i \mid v)-\epsilon)[\right.\right.\right.
$$

$\mathrm{e}$

$$
K(a \mid v)=J(a \mid \emptyset) \cup J(a \mid v) .
$$

Para todo $a \in A-\mathcal{E}=\{\# \mathcal{E}+1, \# \mathcal{E}+2, \ldots\}$

$$
J(a \mid v)=K(a \mid v)=\left[\sum_{i=1}^{a-1} p(i \mid v), \sum_{i=1}^{a} p(i \mid v)[.\right.
$$


Observe que para todo $a \in A$ e todo $v \in \tau$

$$
\lambda(K(a \mid v))=p(a \mid v)
$$

em que $\lambda$ denota a medida de Lebesgue sobre [0,1[. Esta partição de $[0,1[$ é ilustrada na Figura 2.2 .

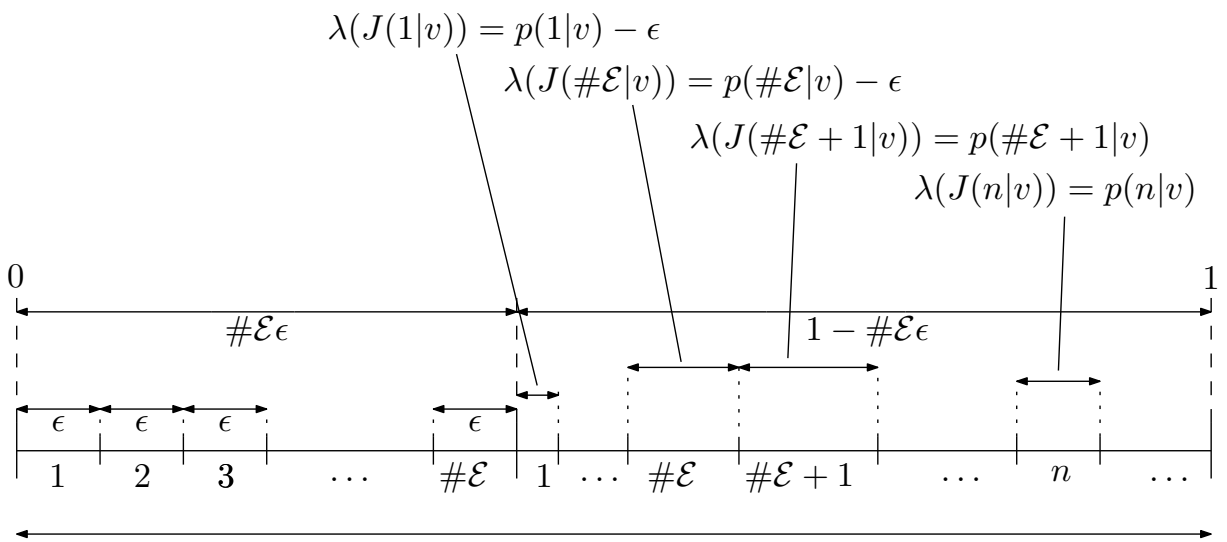

$[0,1[$

Figura 2.2: Ilustração da partição de $[0,1[$.

Definimos a função $F:\left[0,1\left[\times A^{-\mathbb{N}} \rightarrow A\right.\right.$ por

$$
F\left(u_{n}, a_{-\infty}^{n-1}\right)=\sum_{a \in A} a .1\left\{u_{n} \in K\left(a \mid c_{\tau}\left(a_{-\infty}^{n-1}\right)\right)\right\}
$$

que é de particular importância para nossa abordagem construtiva. A função $F$ é uma função mensurável porque $\mathbf{1}\{u \in B\}$ é mensurável quando $B$ é mensurável. No nosso caso, os intervalos $\left\{K\left(a \mid c_{\tau}\left(a_{-\infty}^{n-1}\right)\right)\right\}_{a \in A, a_{-\infty}^{-1} \in A^{-\mathbb{N}}}$ são mensuráveis porque para todo $a$, a função $p(a \mid \cdot)$ é mensurável.

Definição 2.11 (Cadeia com passado fixado). Definimos para todo $\mathbf{a} \in A^{\mathbb{Z}}$ e todo $i \in \mathbb{Z}$, a função $\mathbf{X}^{\mathbf{a}, i}:\left[0,1\left[^{\mathbb{Z}} \rightarrow A^{\mathbb{Z}}, \mathbf{U} \mapsto \mathbf{X}(\mathbf{U})^{\mathbf{a}, i}\right.\right.$, onde $\mathbf{X}(\mathbf{U})^{\mathbf{a}, i}$ é definida por $(X(\mathbf{U}))_{n}^{\mathbf{a}, i}=a_{n}$ para todo $n<i, e$ para $n \geq i$

$$
\left.(X(\mathbf{U}))_{n}^{\mathbf{a}, i}=F\left(U_{n},\left((X(\mathbf{U}))^{\mathbf{a}, i}\right)_{-\infty}^{n-1}\right)\right)
$$

Estas cadeias estocásticas são chamadas cadeias com passado fixado.

Esta função é sempre bem definida pois o passado é fixado. Para todo a e todo $i$ a função $\mathbf{X}(\mathbf{U})^{\mathbf{a}, i}$ é construida de maneira recursiva desde $i$ até $+\infty$. O valor no tempo $n,(X(\mathbf{U}))_{n}^{\mathbf{a}, i}$, para 
todo $n \geq i$, é $\mathcal{F}\left(U_{i}^{n}\right)$-mensurável pela Definição 2.6. Portanto, a função inteira é mensurável.

Por outro lado, temos por definição que para $n \geq i$, estas cadeias têm as probabilidades de transição que são dadas pela árvore $(\tau, p)$ por $(2.5)$. No entanto, elas não são as cadeias compatíveis

$\operatorname{com}(\tau, p)$ por causa do passado que fixamos. O problema da existência de uma cadeia compatível $\operatorname{com}(\tau, p)$ é justamente obter uma cadeia $\mathbf{X}(\mathbf{U})$ tal que para todo $n$,

$$
(X(\mathbf{U}))_{n}=F\left(U_{n},\left((X(\mathbf{U}))_{-\infty}^{n-1}\right)\right)
$$

sem precisar de passado fixado. Vemos depois como podemos mostrar a existência (Seção 2.5) ou a não existência (Seção 3.1) de tais cadeias utilizando estas cadeias com passado fixado.

\subsection{A simulação perfeita e suas consequências}

Fazer uma simulação perfeita significa construir uma amostra finita da cadeia distribuída exatamente de acordo com a medida invariante. Propp \& Wilson (1996) foram os primeiros a realizar uma simulação perfeita de uma cadeia de Markov. Essa idéia deve ser colocada em contraste com a convergência para a medida invariante partindo de qualquer medida inicial, caso no qual a cadeia não está necessariamente na medida invariante. Pode se provar que quando é possível fazer uma simulação perfeita, então a medida invariante existe (por construção), e é única. A ideia de Propp \& Wilson (1996) foi depois generalizada por Comets et al. (2002) no caso das cadeias de alcance infinito, utilizando uma construção baseada sobre um esquema regenerativo introduzido em Ferrari et al. (2000). Informalmente, uma cadeia de alcance infinito tem um equema regenerativo quando existem infinitos pontos ao longo da sequência que dividem a sequência em blocos independentes. A noção de simulação pefeita está relacionada à noção de esquema regenerativo (embora Comets et al. (2002) mostraram que pode ser feita a simulação perfeita sem ter esquema regenerativo), pois os pontos de regeneração podem ser utilizados como ponto de partida para se construir a cadeia. A existênica de um esquema regenerativo já havia sido provada por Lalley (1986) quando $\beta_{k}$ decai exponencialmente rápido.

Nesta seção vamos explicar como funciona a nossa simulação perfeita. Fazemos uma primeira parte explicativa e um pouco informal. Em seguida introduzimos formalmente todas as grandezas envolvidas no algoritmo de simulação perfeita. 


\subsubsection{Introdução informal}

Definição 2.12 (Simulação perfeita). Dada uma família de probabilidades de transição, uma simulação perfeita é um algoritmo capaz de construir uma amostra distribuída exatamente de acordo com a lei estacionária compatível com esta família de probabilidades de transição, sem conhecer a priori esta lei estacionária.

Este tipo de construção pode ser feito utilizando um acoplamento desde o passado (coupling from the past em inglês). No nosso caso, esta construção é similar à construção da seção anterior, mas sem precisar do passado fixado. Propomos descrever primeiro informalmente este procedimento.

Suponha que a árvore probabilística $(\tau, p)$ seja tal que $\mathcal{E} \neq \emptyset$. Neste caso, utilizando a mesma construção definida na seção precedente, podemos ver que para todo passado fixado a e todo $i$, sempre que $U_{n}, n \geq i$, pertence ao intervalo $J(a \mid \emptyset)$ para algum $a$ em $\mathcal{E}$, colocamos $(X(\mathbf{U}))_{n}^{\mathbf{a}, i}=a$. Portanto, para estes tempos $n$, não precisamos do passado e dizemos que são tempos auto-definidos. Se além disso $(\tau, p)$ possui um contexto $w$ cujas letras estão em $\mathcal{E}$, sabemos (por ergodicidade) que teremos infinitas vezes $U_{n-j+1} \in J\left(w_{-j} \mid \emptyset\right), j=1, \ldots,|w|$, isto é para infinitos tempos $n$ :

$$
\left((X(\mathbf{U}))^{\mathbf{a}, i}\right)_{n-|w|+1}^{n}=w
$$

independentemente de a. Se $n$ é um tempo tal que isso acontece, podemos construir também $(X(\mathbf{U}))_{n+1}$ sem precisar conhecer o passado $\left((X(\mathbf{U}))_{j}^{\mathbf{a}, i}\right)_{j \leq n-|w|+1}$ (aqui utilizamos a propriedade das cadeias de alcance variável, isto é o fato que $w$ é um contexto de $\tau$, e portanto não precisamos do passado mais remoto para escolher o próximo símbolo): colocamos

$$
(X(\mathbf{U}))_{n+1}^{\mathbf{a}, i}=\sum_{a \in A} a .1\left\{U_{n+1} \in K(a \mid w)\right\}
$$

Segundo o símbolo $a_{n+1}$ que colocamos no tempo $n+1$, e segundo a forma de $\tau$, pode ser que $w a_{n+1}$ seja um elemento de $\tau$, e neste caso, podemos continuar a construção de $(X(\mathbf{U}))_{n+2}^{\mathbf{a}, i}$, independentemente de a. Se não for o caso, ainda é possível que o tempo $n+2$ seja auto-definido, e neste caso também podemos continuar a construção. Suponha que este procedimento nos levou até o tempo $n+l$ sem nunca precisar conhecer $(X(\mathbf{U}))_{-\infty}^{n-|w|+1}$ (e portanto, independentemente de a), 
neste caso, teremos construído uma sequência $(X(\mathbf{U}))_{n-|w|+1}^{n+l}$ tal que

$$
(X(\mathbf{U}))_{n-|w|+1}^{n+l}=\left((X(\mathbf{U}))^{\mathbf{a}, i}\right)_{n-|w|+1}^{n+l}, \quad \forall \mathbf{a} \in A^{\mathbb{Z}}
$$

utilizando unicamente $U_{n-|w|+1}^{n+l}$ e a informação dada por $(\tau, p)$.

O acoplamento desde o passado se faz da maneira seguinte: suponha que queremos construir uma amostra da cadeia compatível com $(\tau, p)$ sobre um intervalo de tempo $m, \ldots, n$ com $-\infty<$ $m \leq n<+\infty$, e distribuída exatamente na medida estacionária. Engendramos $U_{\theta[m, n]}, \ldots, U_{n}$, onde $\theta[m, n]$ (possivelmente igual a $-\infty$ ) é o maior tempo inferior ou igual a $m$ tal que pudemos reconstruir $(X(\mathbf{U}))_{\theta[m, n]}^{n}$ utilizando $U_{\theta[m, n]}^{n}$ com o procedimento descrito informalmente acima. A questão de saber se podemos fazer uma simulação perfeita, isto é, se o que acabamos de dizer é factível, se resolve mostrando que para todo intervalo de tempo $m, \ldots, n$ com $-\infty<m \leq n<+\infty$, o tempo $\theta[m, n]$ é $\mathbb{P}$-q.c. finito. Neste caso, podemos mostrar que a amostra engendrada é de fato uma amostra da cadeia estacionária compatível com $(\tau, p)$.

\subsubsection{O Algoritmo}

Primeiro, observamos que na construção apresentada anteriormente, precisamos que para algum contexto $w$ de $\tau$, todas as letras aparecendo em $w$ sejam $\epsilon$-regulares para algum $\epsilon>0$. Se denotamos por $\mathcal{W}$ o subconjunto de $A$ formado pelos símbolos aparecendo em $w$, precisamos que $\mathcal{W} \subset \mathcal{E}$. Agora, definimos de maneira formal o que é a variável aleatória $\theta[m, n]$ para todo $-\infty<m \leq n \leq+\infty$. Para isso precisamos de duas funções. Primeiro, uma extensão da função $F$ (introduzida na seção anterior) para elementos de $A^{\star}: F:\left[0,1\left[\times\left(A^{\star} \cup A^{-\mathbb{N}}\right) \rightarrow A \cup\{\star\}\right.\right.$

$$
F\left(u, a_{m}^{n}\right)=\sum_{a \in A} a . \mathbf{1}\left\{u \in K\left(a \mid c_{\tau}\left(a_{m}^{n}\right)\right)\right\}+\star \mathbf{1}\left\{u \in \left[\# \mathcal{E} \epsilon, 1\left[, c_{\tau}\left(a_{m}^{n}\right)=\emptyset\right\}, \forall m \leq n+1,\right.\right.
$$

que utilizaremos para construir a cadeia. Lembramos o seguinte fato sobre a notação: no caso em que $m=n+1$, adotamos por convenção que $a_{m}^{n}=a_{n+1}^{n}:=\emptyset$. Supondo que já construímos $(X(\mathbf{U}))_{m}^{n}=a_{m}^{n}$, tentamos construir $(X(\mathbf{U}))_{n+1}$ utilizando $F:$ se $F\left(U_{n+1}, a_{m}^{n}\right)=a \in A$, então fazemos $(X(\mathbf{U}))_{n+1}=a$, caso contrário, $F\left(U_{n+1}, a_{m}^{n}\right)=\star$ e significa que não podemos construir $(X(\mathbf{U}))_{n+1}$ conhecendo somente $(X(\mathbf{U}))_{m}^{n}=a_{m}^{n}$ porque não há sufixo de $a_{m}^{n}$ que seja contexto, e 
$U_{n+1} \notin[0, \# \mathcal{E} \epsilon[$. Aqui está a diferença com a construção sobre um passado fixado. Às vezes, não podemos determinar o próximo símbolo, porque não temos informação suficiente sobre o passado da cadeia. Isso nunca acontece quando fixamos um passado a. No entanto, observamos que quando $c_{\tau}\left(a_{m}^{n}\right) \neq \emptyset$ (o que significa que há um sufixo de $a_{m}^{n}$ que é contexto), então

$$
\mathbb{P}\left(F\left(U_{n+1}, a_{m}^{n}\right)=a\right)=\mathbb{P}\left(U_{n+1} \in K\left(a \mid c_{\tau}\left(a_{m}^{n}\right)\right)\right)=\lambda\left(K\left(a \mid c_{\tau}\left(a_{m}^{n}\right)\right)\right)=p\left(a \mid c_{\tau}\left(a_{m}^{n}\right)\right) .
$$

Isso significa que procedendo deste modo estamos construíndo uma cadeia cujas probabilidades de transição são aquelas dadas pela árvore probabilística $(\tau, p)$. Está função é bem definida e mensurável pelas mesmas razões que antes, acrescentando o fato que pela definição de $\tau$, o evento $\left\{c_{\tau}\left(a_{m}^{n}\right)=\emptyset\right\}$ é $\mathcal{F}\left(a_{m}^{n}\right)$-mensurável.

A segunda função a ser introduzida é $\mathcal{L}:\left[0,1\left[^{n-m+1} \rightarrow\{0,1\}\right.\right.$, que é $\mathcal{F}\left(U_{m}^{n}\right)$-mensurável:

$$
\mathcal{L}\left(U_{m}^{n}\right):=\mathbf{1}\left\{\bigcap_{i=m}^{n} \bigcup_{a_{i} \in A}\left\{F\left(U_{i}, a_{m}^{i-1}\right)=a_{i}\right\}\right\}
$$

Em palavras, esta função tem valor 1 quando podemos construir $(X(\mathbf{U}))_{m}^{n}$ utilizando somente $U_{m}^{n}$ com o procedimento que descrevemos informalmente acima. Uma observação importante: para conseguir construir o valor da cadeia no tempo $m$ utilizando somente $U_{m}$ temos

$$
\begin{gathered}
\mathcal{L}\left(U_{m}\right):=\mathbf{1}\left\{\bigcup_{a_{m} \in A}\left\{F\left(U_{m}, a_{m}^{m-1}\right)=a_{m}\right\}\right\}:=\mathbf{1}\left\{\bigcup_{a_{m} \in A}\left\{F\left(U_{m}, \emptyset\right)=a_{m}\right\}\right\} \\
=\mathbf{1}\left\{\bigcup_{a_{m} \in A}\left\{U_{m} \in K\left(a_{m} \mid \emptyset\right)\right\}\right\}
\end{gathered}
$$

que, pela definição dos intervalos dada na seção precedente, vale $\mathbf{1}\left\{U_{m} \in[0, \# \mathcal{E} \epsilon[\}\right.$. Esses são justamente os tempos que chamamos de auto-definidos. Agora, a definição formal de $\theta[m, n]$ é muito simples: para todo $-\infty<m \leq n \leq+\infty$

$$
\theta[m, n]:=\max \left\{k \leq m: \mathcal{L}\left(U_{k}^{n}\right)=1\right\}
$$

É claro pela definição que esta variável é um tempo de parada no passado sobre a sequência $\left(U_{i}\right)_{i \leq n}$ 
e portanto $\{\theta[m, n]=k\}$ é $\mathcal{F}\left(U_{k}^{n}\right)$-mensurável. Escreveremos as vezes $\theta[m, n](\mathbf{U})$ para lembrar que $\theta[m, n]$ é uma função de $\mathbf{U}$. Também usaremos a notação $\theta[n]:=\theta[n, n]$. Pela definição (2.9), temos para todo $-\infty<m \leq n \leq+\infty$

$$
\theta[m] \geq \theta[m, n] \geq \theta[m+\infty]
$$

e também para todo $i, \theta[m, n] \leq i \leq n$

$$
\theta[m, n] \leq \theta[i]
$$

Apresentamos agora um algoritmo que constroi uma amostra $(X(\mathbf{U}))_{m}^{n}$ para qualquer $-\infty<m \leq$ $n<+\infty$, encontrando $\theta[m, n]$. De fato, este algoritmo retorna também $(X(\mathbf{U}))_{\theta[m, n]}^{m-1}$, que vem "de graça". Ele utiliza as variáveis $i$ e $j$ que são índices de tempo a valor no conjunto $\{\ldots, m-2, m-$ $1, m\}$.

Quando podemos mostrar que $\theta[m, n]$ é $\mathbb{P}$-q.c. finito para todo $-\infty<m \leq n<+\infty$ o algoritmo acima retorna num tempo finito a amostra de uma cadeia $(X(\mathbf{U}))_{m}^{n}$. No entanto, não provamos ainda que esta amostra é uma amostra da cadeia compatível com a nossa árvore probabilística $(\tau, p)$ na medida estacionária, nem que esta cadeia é única. Mostramos isso na Seção 2.5.4, seguindo os argumentos de Comets et al. (2002). Mas antes, fazemos uma seção explicativa, onde apresentamos um exemplo concreto de árvore de contextos probabilística, e de simulação perfeita da cadeia compatível com esta árvore.

\subsubsection{Um exemplo de simulação perfeita}

Consideramos a árvore probabilística $(\tau, p)$ da Figura 2.3. 


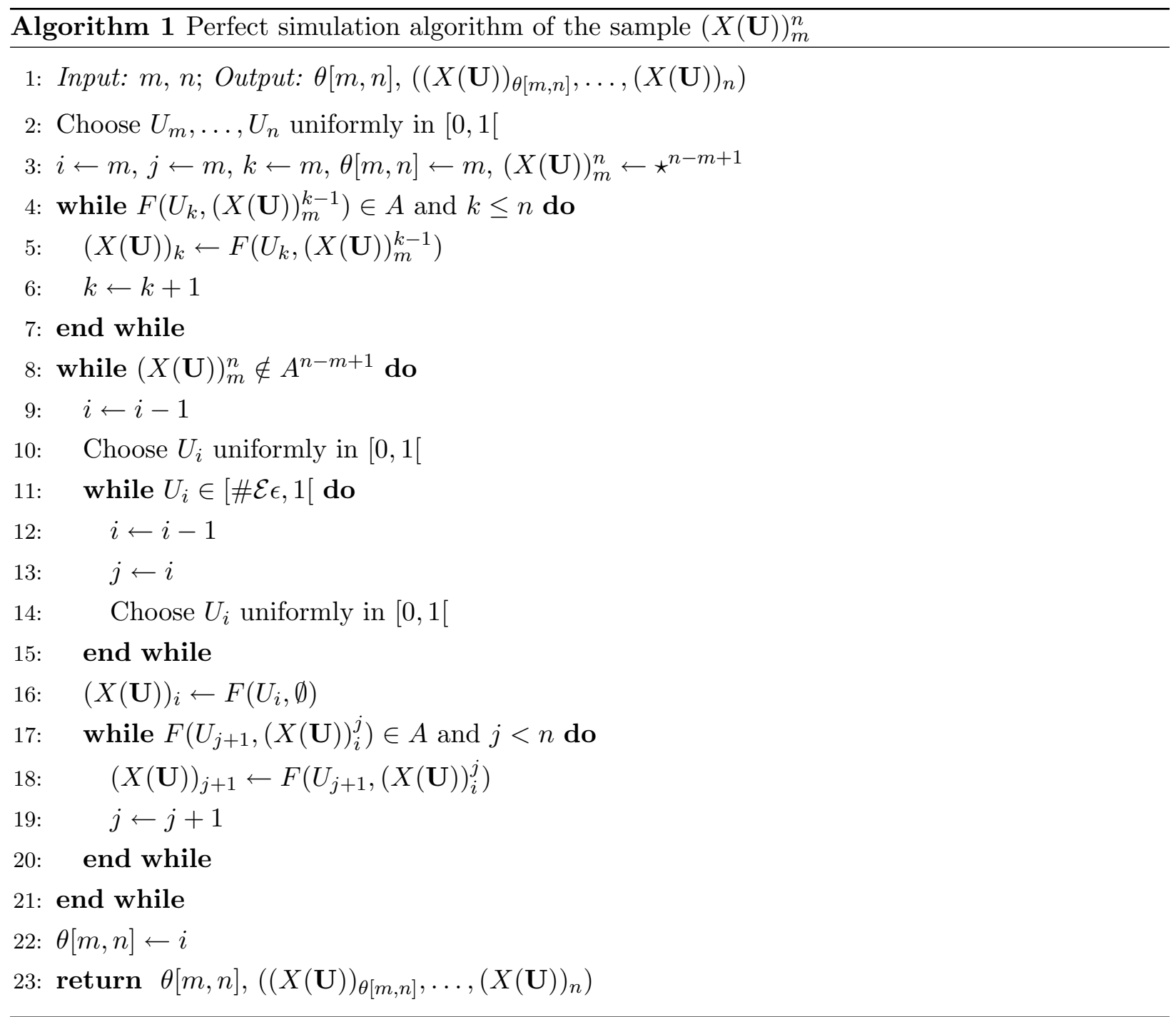

As probabilidades de transição são tais que os símbolos 0 e 1 são ambos $\epsilon$-regulares com $\epsilon=$ 0,2. Não especificamos todas as probabilidades de transição pois seria um pouco exaustivo... Consideramos uma realização da cadeia $\mathbf{U}$, e queremos construir $(X(\mathbf{U}))_{n}$ (ver a Figura 2.4). Para a construção, precisamos de várias probabilidades de transição, portanto, o leitor não tem toda a informação para acompanhar a Figura 2.4. Só pode acompanhar se $U_{i}$ pertence ou não ao intervalo $[0, \# \mathcal{E} \epsilon[$, e acompanhar os contextos que aparecem ao longo dos passos do algoritmo. Se ocorre o símbolo "?" num passo $l$ do algoritmo, isso indica que a informação que obtivemos até este passo não nos permite escolher o símbolo da cadeia $(X(\mathbf{U}))_{t(l)}$ (onde $t(l)$ é o tempo que estamos tentando reconstruir neste passo do algoritmo). Lembramos que isso ocorre quando $U_{t(l)}$ pertence a $[\# \mathcal{E} \epsilon, 1[=[0,4,1[$, e que não há contexto na amostra de $\mathbf{X}(\mathbf{U})$ que conseguimos contruir até este 


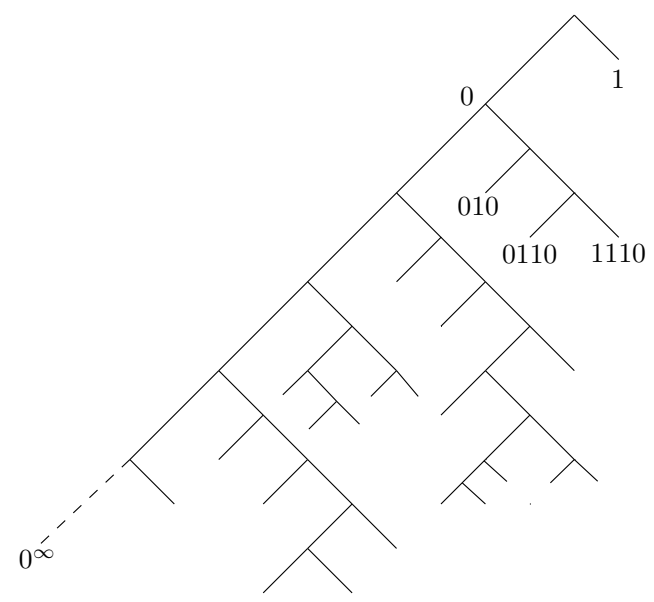

Figura 2.3: Árvore sobre $A=\{0,1\}$ tendo 1 como bom contexto

passo do algoritmo.

Podemos verificar que este é o caso no passo $i=12$ na Figura 2.4. Temos $U_{t(i)}=U_{n-5}=0,42$, e só conseguimos construir (até o passo $i)(X(\mathbf{U}))_{n-1}=0,(X(\mathbf{U}))_{n-2}=1,(X(\mathbf{U}))_{n-6}=0$ e $(X(\mathbf{U}))_{n-7}=1$. Portanto, $c_{\tau}\left((X(\mathbf{U}))_{n-7}(X(\mathbf{U}))_{n-6}\right)=c_{\tau}(10)=\emptyset$. Por esta razão, o tempo $n-5$ é "?" na etapa $i$. Somente na etapa $j$, podemos reconstruir $(X(\mathbf{U}))_{n-5}$ porque nesta etapa, já conseguimos construir $(X(\mathbf{U}))_{t(j)-13}^{t(j)-1}$ :

$$
(X(\mathbf{U}))_{t(j)-13}^{t(j)-1}=1101011100110, \quad \text { e } c_{\tau}\left((X(\mathbf{U}))_{t(j)-13}^{t(j)-1}\right)=c_{\tau}(1101011100110)=0110 .
$$

\subsubsection{Consequências: existência e unicidade}

Teorema 2.13. (Existência, unicidade e perda de memória). Quando $\theta[m, n]$ é $\mathbb{P}$-q.c. finito para todo $-\infty<m \leq n<+\infty$, Algoritmo 1 acima retorna uma amostra da única cadeia estacionária compativel com $(\tau, p)$. Também, a taxa de perda de memória da cadeia compatível $\mathbf{X}(\mathbf{U})$ verifica para $i \leq 0$

$$
\left|\mu\left((X(\mathbf{U}))_{0}\right)-\mu\left((X(\mathbf{U}))_{0} \mid X_{n}=x_{n}, n<i\right)\right| \leq \mathbb{P}(\theta[0]<i) .
$$

Definimos para todo $-\infty<m \leq n<+\infty$ o conjunto

$$
\Omega_{[m, n]}=\{\mathbf{U} \in[0,1[\mathbb{Z}: \theta[m, n](\mathbf{U})>-\infty\} .
$$

que é $\mathcal{F}\left(U_{-\infty}^{n}\right)$-mensurável. Sobre $\Omega_{[n]}$, o algoritmo acima retorna uma amostra $(X(\mathbf{U}))_{\theta[n]}, \ldots,(X(\mathbf{U}))_{n}$ 


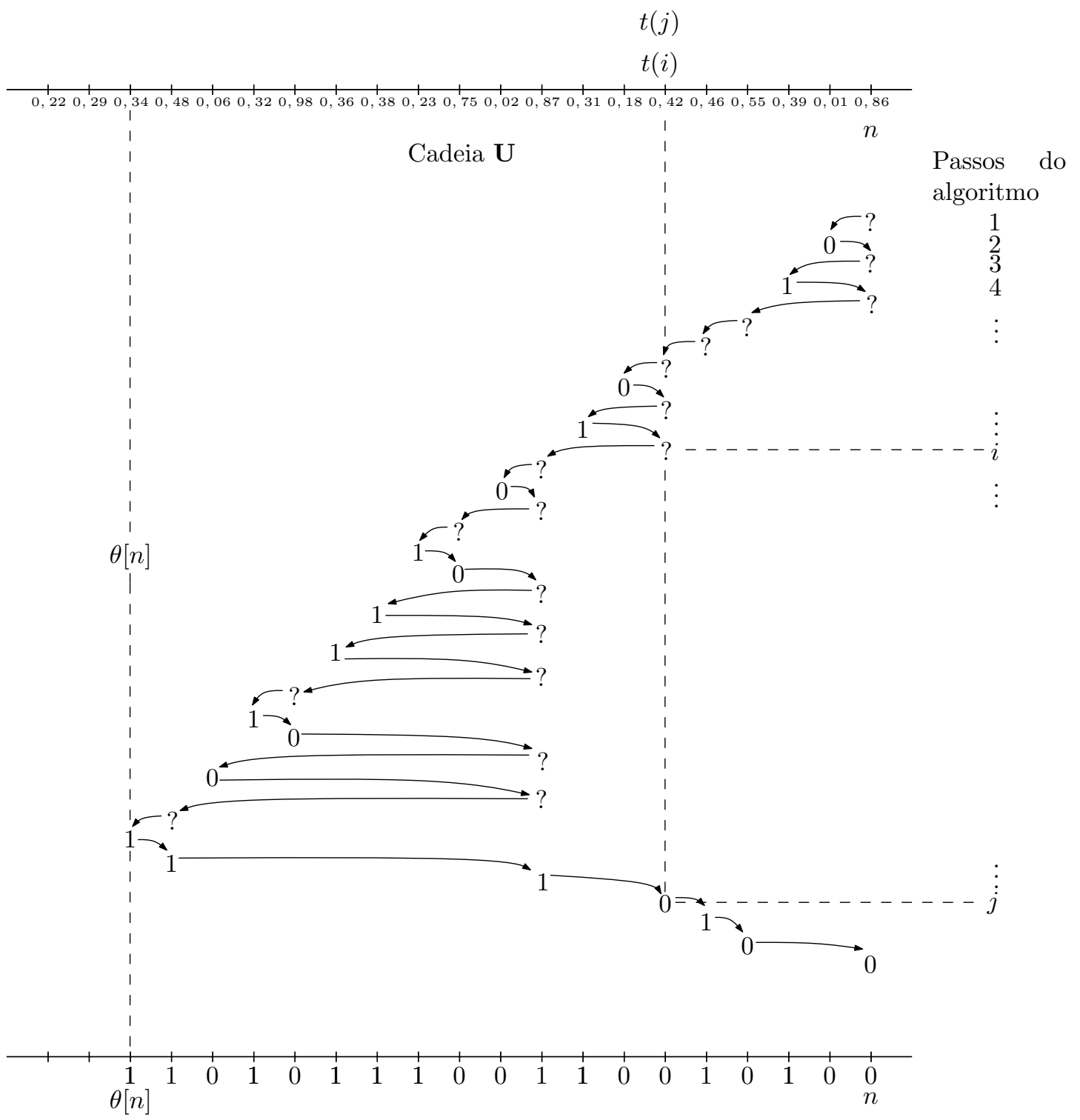

Cadeia X

Figura 2.4: Exemplo de simulação perfeita da cadeia compatível com a árvore da Figura 2.3.

definida recursivamente para $\theta[n] \leq i \leq n$, por

$$
(X(\mathbf{U}))_{i}=F\left(U_{i},(X(\mathbf{U}))_{\theta[n]}^{i-1}\right) \in A .
$$

A consistência desta construção segue do fato que $\theta[n] \leq \theta[i]$ para $\theta[n] \leq i \leq n$ e, portanto, o resultado obtido na recursão acima no tempo $i$ é o mesmo que o resultado obtido se estivéssemos calculando $(X(\mathbf{U}))_{i}$. Também, quando $\theta[m, n]$ é quase-certamente finito para todo $m \leq n$, podemos construir a cadeia inteira $\mathbf{X}(\mathbf{U})$ como uma função determinística $X:\left[0,1\left[\mathbb{Z} \rightarrow A^{\mathbb{Z}}, \mathbf{U} \mapsto \mathbf{X}(\mathbf{U})\right.\right.$, 
atribuíndo para todo $i$ em $\mathbb{Z}$, o valor $(X(\mathbf{U}))_{i}=F\left(U_{i},(X(\mathbf{U}))_{-\infty}^{i-1}\right)$. Esta função pode ser vista como uma concatenação de funções $(X(\mathbf{U}))_{i}$, que são todas $\mathcal{F}\left(U_{-\infty}^{i}\right)$-mensurável sobre $\Omega_{[i]}$ como acabamos de observar, e como para todo $i, \Omega_{[i]}$ também é $\mathcal{F}\left(U_{-\infty}^{i}\right)$-mensurável, a função $\mathbf{X}(\mathbf{U})$ toda é mensurável.

Prova da compatibilidade. Precisamos mostrar que a lei $\mathbb{P}(\mathbf{X}(\mathbf{U}) \in \cdot)$ é compatível com $(\tau, p)$, ou em outras palavras, que $\mathbf{X}(\mathbf{U})$ verifica (2.4). Para mostrar isso, vamos trabalhar sobre o conjunto

$$
\Omega_{[0]}=\left\{\mathbf { U } \in \left[0,1\left[^{\mathbb{Z}}: \theta[0](\mathbf{U})>-\infty\right\}\right.\right.
$$

Temos

$$
\mathbb{E}\left(\mathbf{1}\left\{(X(\mathbf{U}))_{0}=a\right\} \mid U_{-\infty}^{-1}\right)=\mathbb{E}\left(\mathbf{1}\left\{U_{0} \in K\left(a \mid c_{\tau}\left((X(\mathbf{U}))_{-\infty}^{-1}\right)\right)\right\} \mid U_{-\infty}^{-1}\right)
$$

e como $(X(\mathbf{U}))_{-\infty}^{-1}$ é $\mathcal{F}\left(U_{-\infty}^{-1}\right)$-mensurável e $U_{0}$ não depende de $\mathcal{F}\left(U_{-\infty}^{-1}\right)$, a equação acima torna-se

$$
\mathbb{E}\left(\mathbf{1}\left\{(X(\mathbf{U}))_{0}=a\right\} \mid U_{-\infty}^{-1}\right)=\mathbb{E}\left(\mathbf{1}\left\{U_{0} \in K\left(a \mid c_{\tau}\left((X(\mathbf{U}))_{-\infty}^{-1}\right)\right)\right\}\right)=p\left(a \mid c_{\tau}\left((X(\mathbf{U}))_{-\infty}^{-1}\right)\right),
$$

em que a última igualdade segue de (2.8). Isso significa em particular que a parte da esquerda é $\mathcal{F}\left((X(\mathbf{U}))_{-\infty}^{-1}\right)$-mensurável pois a parte da direita só depende de $(X(\mathbf{U}))_{-\infty}^{-1}$, segue que

$$
\mathbb{E}\left(\mathbf{1}\left\{(X(\mathbf{U}))_{0}=a\right\} \mid U_{-\infty}^{-1}\right)=\mathbb{E}\left(\mathbb{E}\left(\mathbf{1}\left\{(X(\mathbf{U}))_{0}=a\right\} \mid U_{-\infty}^{-1}\right) \mid(X(\mathbf{U}))_{-\infty}^{-1}\right)=p\left(a \mid c_{\tau}\left((X(\mathbf{U}))_{-\infty}^{-1}\right)\right) .
$$

Como $\mathcal{F}\left((X(\mathbf{U}))_{-\infty}^{-1}\right) \subset \mathcal{F}\left(U_{-\infty}^{-1}\right)$, obtemos $\mathbb{E}\left(\mathbf{1}\left\{(X(\mathbf{U}))_{0}=a\right\} \mid(X(\mathbf{U}))_{-\infty}^{-1}\right)=p\left(a \mid c_{\tau}\left((X(\mathbf{U}))_{-\infty}^{-1}\right)\right)$, em outras palavras

$$
\mathbb{P}\left((X(\mathbf{U}))_{0}=a \mid(X(\mathbf{U}))_{-\infty}^{-1}\right)=p\left(a \mid c_{\tau}\left((X(\mathbf{U}))_{-\infty}^{-1}\right)\right)
$$

que vale $\mathbb{P}$-q.c. quando $\Omega_{[0]}$ tem medida 1 .

Prova da estacionariedade. Para provar a estacionariedade mostramos que a função $\mathbf{U} \mapsto \mathbf{X}(\mathbf{U})$ é equivariante à translação (translation-equivariant). Isto é, se $T:\left[0,1\left[^{\mathbb{Z}} \rightarrow\left[0,1 \mathbb{Z}^{\mathbb{Z}}\right.\right.\right.$ denota o operador deslocamento $(T \mathbf{U})_{n}=U_{n+1}$, queremos mostrar que para todo $n$ em $\mathbb{Z}$

$$
(X(T \mathbf{U}))_{n}=(X(\mathbf{U}))_{n+1}
$$


Para simpificar a notação escrevemos $\mathbf{U}^{\prime}$ para $T \mathbf{U}\left(\operatorname{assim} U_{n}^{\prime}=U_{n+1}\right)$ e $\theta^{\prime}[n]$ para $\theta\left(\mathbf{U}^{\prime}\right)[n]$. Queremos calcular

$$
\left(X\left(\mathbf{U}^{\prime}\right)\right)_{n}:=\sum_{a \in A} a . \mathbf{1}\left\{U_{n}^{\prime} \in K\left(a \mid c_{\tau}\left(\left(X\left(\mathbf{U}^{\prime}\right)\right)_{\theta^{\prime}[n]}^{n-1}\right)\right)\right\}
$$

Primeiro, é claro que $\theta[n+1]=m+1 \Leftrightarrow \theta^{\prime}[n]=m$ pois $U_{m+1}^{n+1}=\left(U^{\prime}\right)_{m}^{n}$. Em outras palavras

$$
\theta^{\prime}[n]=\theta[n+1]-1
$$

e (2.10) torna-se

$$
\left(X\left(\mathbf{U}^{\prime}\right)\right)_{n}=\sum_{a \in A} a . \mathbf{1}\left\{U_{n+1} \in K\left(a \mid c_{\tau}\left(\left(X\left(\mathbf{U}^{\prime}\right)\right)_{\theta[n+1]-1}^{n-1}\right)\right)\right\}
$$

Por outro lado, $U_{\theta^{\prime}[n]}^{\prime}=U_{\theta[n+1]}$, portanto, como esses tempos são auto-definidos

$$
\left(X\left(\mathbf{U}^{\prime}\right)\right)_{\theta^{\prime}[n]}=(X(\mathbf{U}))_{\theta[n+1]},
$$

o que significa que

$$
\left(X\left(\mathbf{U}^{\prime}\right)\right)_{\theta[n+1]-1}=(X(\mathbf{U}))_{\theta[n+1]}
$$

Vamos proceder por iteração e supor que $\left(X\left(\mathbf{U}^{\prime}\right)\right)_{i}=(X(\mathbf{U}))_{i+1}$ para $i=\theta[n+1]-1, \ldots, j-1$ com $j \leq n$. No tempo $j$ temos

$$
\left(X\left(\mathbf{U}^{\prime}\right)\right)_{j}:=\sum_{a \in A} a .1\left\{U_{j}^{\prime} \in K\left(a \mid c_{\tau}\left(\left(X\left(\mathbf{U}^{\prime}\right)\right)_{\theta^{\prime}[j]}^{j-1}\right)\right)\right\}
$$

com $\theta^{\prime}[j] \geq \theta^{\prime}[n]$ pois $j \leq n$ (isso segue da definição (2.9)). Então

$$
\begin{aligned}
& \left(X\left(\mathbf{U}^{\prime}\right)\right)_{j}=\sum_{a \in A} a \cdot \mathbf{1}\left\{U_{j+1} \in K\left(a \mid c_{\tau}\left(\left(X\left(\mathbf{U}^{\prime}\right)\right)_{\theta[j+1]-1}^{j-1}\right)\right)\right\} \\
= & \sum_{a \in A} a . \mathbf{1}\left\{U_{j+1} \in K\left(a \mid c_{\tau}\left((X(\mathbf{U}))_{\theta[j+1]}^{j}\right)\right)\right\}=(X(\mathbf{U}))_{j+1} .
\end{aligned}
$$

Portanto $\left(X\left(\mathbf{U}^{\prime}\right)\right)_{\theta[n+1]-1}^{n-1}=(X(\mathbf{U}))_{\theta[n+1]}^{n}$, e (2.10) torna se

$$
\left(X\left(\mathbf{U}^{\prime}\right)\right)_{n}=\sum_{a \in A} a . \mathbf{1}\left\{U_{n+1} \in K\left(a \mid c_{\tau}\left((X(\mathbf{U}))_{\theta[n+1]}^{n}\right)\right)\right\}=(X(\mathbf{U}))_{n+1}
$$


Resumindo, mostramos que $\mathbf{X}(\mathbf{U})$ é obtido como uma função equivariante à translação de uma cadeia estacionária, portanto a cadeia $\mathbf{X}(\mathbf{U})$ é ela também estacionária.

Prova da unicidade. Suponhamos que $\mu$ e $\mu^{\prime}$ são duas medidas estacionárias sobre $A^{\mathbb{Z}}$ compatíveis $\operatorname{com}(\tau, p)$, temos:

$$
\begin{aligned}
\left|\mu\left(x_{0}\right)-\mu^{\prime}\left(x_{0}\right)\right|=\mid \int d \mu(\mathbf{y}) \mu\left(X_{0}=x_{0} \mid X_{n}=y_{n}, n<i\right) & \\
& -\int d \mu^{\prime}\left(\mathbf{y}^{\prime}\right) \mu^{\prime}\left(X_{0}=x_{0} \mid X_{n}=y_{n}^{\prime}, n<i\right) \mid .
\end{aligned}
$$

Pela Definição 2.11 sobre as cadeias com passado fixado, quando $\mu$ e $\mu^{\prime}$ são compatíveis com $(\tau, p)$ temos

$$
\mathbb{P}\left((X(\mathbf{U}))_{0}^{\mathbf{y}, i}=x_{0}\right)=\mu\left(X_{0}=x_{0} \mid X_{n}=y_{n}, n<i\right)
$$

e

$$
\mathbb{P}\left((X(\mathbf{U}))_{0}^{\mathbf{y}^{\prime}, i}=x_{0}\right)=\mu^{\prime}\left(X_{0}=x_{0} \mid X_{n}=y_{n}^{\prime}, n<i\right)
$$

portanto

$$
\begin{array}{r}
\left|\mu\left(x_{0}\right)-\mu^{\prime}\left(x_{0}\right)\right|=\mid \int d \mu(\mathbf{y}) \mathbb{P}\left(\left(\left.X(\mathbf{U})\right|_{0} ^{\mathbf{y}, i}=x_{0}\right)-\int d \mu^{\prime}\left(\mathbf{y}^{\prime}\right) \mathbb{P}\left((X(\mathbf{U}))_{0}^{\mathbf{y}^{\prime}, i}=x_{0}\right) \mid\right. \\
=\left|\iint d \mu(\mathbf{y}) d \mu^{\prime}\left(\mathbf{y}^{\prime}\right)\left(\mathbb{P}\left((X(\mathbf{U}))_{0}^{\mathbf{y}, i}=x_{0}\right)-\mathbb{P}\left((X(\mathbf{U}))_{0}^{\mathbf{y}^{\prime}, i}=x_{0}\right)\right)\right| \\
\leq \iint d \mu(\mathbf{y}) d \mu^{\prime}\left(\mathbf{y}^{\prime}\right) \mid \mathbb{P}\left(\left(\left.X(\mathbf{U})\right|_{0} ^{\mathbf{y}, i}=x_{0}\right)-\mathbb{P}\left((X(\mathbf{U}))_{0}^{\mathbf{y}^{\prime}, i}=x_{0}\right) \mid\right.
\end{array}
$$

Como as duas cadeias estão construídas sobre o mesmo espaço de probabilidade $(\Omega, \mathcal{F}, \mathbb{P})$ obtemos

$$
\left|\mu\left(x_{0}\right)-\mu^{\prime}\left(x_{0}\right)\right| \leq \iint d \mu(\mathbf{y}) d \mu^{\prime}\left(\mathbf{y}^{\prime}\right)\left|\mathbb{P}\left((X(\mathbf{U}))_{0}^{\mathbf{y}, i} \neq(X(\mathbf{U}))_{0}^{\mathbf{y}^{\prime}, i}\right)\right|
$$

Mas como $\left\{\left(\left.X(\mathbf{U})\right|_{0} ^{\mathbf{y}, i} \neq\left(\left.X(\mathbf{U})\right|_{0} ^{\mathbf{y}^{\prime}, i}\right\} \subset\{\theta[0]<i\}\right.\right.$, isso implica

$$
\left|\mu\left(X_{0}\right)-\mu^{\prime}\left(X_{0}\right)\right| \leq \mathbb{P}(\theta[0]<i)
$$


que vai para zero quando $i \rightarrow-\infty$ se $\theta[0]$ é $\mathbb{P}$-q.c. finito. O resultado de perda de memória é obtido de maneira similar.

\subsection{Esquema regenerativo}

Denominamos o tempo $t$ tempo de regeneração para a nossa cadeia $\mathbf{X}(\mathbf{U})$ se

$$
\theta[t,+\infty]=t
$$

Defina $\boldsymbol{\xi}$ a cadeia a valor em $\{0,1\}$ dado por

$$
\xi_{j}:=\mathbf{1}\{j=\theta[j,+\infty]\}, j \in \mathbb{Z}
$$

Consideramos a sequência $\mathbf{T}$ de índices de tempo definida de maneira que $\xi_{j}=1$ se e somente se $j=T_{l}$ para algum $l$ de $\mathbb{Z}, T_{l}<T_{l+1}$ e com a convenção que $T_{0} \leq 0<T_{1}$. Dizemos que $\mathbf{X}(\mathbf{U})$ tem um esquema regenerativo se a cadeia $\boldsymbol{\xi}$ é uma cadeia de renovação.

Teorema 2.14. Se para todo $n \in \mathbb{Z}$ temos $\mathbb{P}(\theta[n,+\infty]=n)>0$, então existe um esquema regenerativo em relação aos tempos aleatórios $\theta[m, n]$.

Demonstração. Temos que mostrar que a cadeia $\boldsymbol{\xi}$ é uma cadeia de renovação. Para isso, basta mostrar que para uma sequência qualquer de inteiros $t_{1}<t_{2}, \ldots<t_{n}$, temos

$$
\begin{aligned}
\mathbb{P}\left(\xi_{t_{l}}=1, l=1, \ldots, n\right)= & \mathbb{P}\left(\xi_{0}=1\right) \times \mathbb{P}\left(\xi_{t_{n-1}-t_{n}}=1 \mid \xi_{0}=1\right) \times \ldots \\
& \times \mathbb{P}\left(\xi_{t_{1}-t_{2}}=1 \mid \xi_{0}=1\right) .
\end{aligned}
$$

Pela Definição 2.9 (utilizando a convenção que $t_{n+1}=+\infty$ )

$$
\bigcap_{l=1}^{n}\left\{\theta\left[t_{l},+\infty\right]=t_{l}\right\}=\bigcap_{l=1}^{n}\left\{\theta\left[t_{l}, t_{l+1}-1\right]=t_{l}\right\},
$$

que é uma interseção de eventos independentes, pois $\left\{\theta\left[t_{l}, t_{l+1}-1\right]=t_{l}\right\}$ é $\mathcal{F}\left(U_{t_{l}}^{t_{l+1}-1}\right)$-mensurável 
para $l=1, \ldots, n$. Portanto

$$
\mathbb{P}\left(\xi_{t_{l}}=1, l=1, \ldots, n\right)=\prod_{l=1}^{n} \mathbb{P}\left(\theta\left[t_{l}, t_{l+1}-1\right]=t_{l}\right) .
$$

Por outro lado, como a cadeia $\mathbf{U}$ é invariante por translação, temos

$$
\mathbb{P}\left(\theta\left[t_{n+1},+\infty\right]=t_{n+1}\right)=\mathbb{P}(\theta[0,+\infty]=0)=\mathbb{P}\left(\xi_{0}=1\right),
$$

que é estritamente positivo nas condições do teorema, e para $l=1, \ldots, n-1$

$$
\mathbb{P}\left(\theta\left[t_{l}, t_{l+1}-1\right]=t_{l}\right)=\mathbb{P}\left(\theta\left[t_{l}-t_{l+1},-1\right]=t_{l}-t_{l+1}\right)
$$

Também, analogamente a $(2.11)$, temos para $l=1, \ldots, n-1$

$$
\begin{gathered}
\mathbb{P}\left(\theta\left[t_{l}-t_{l+1},+\infty\right]=t_{l}-t_{l+1} \mid \theta[0,+\infty]=0\right)=\frac{\mathbb{P}\left(\theta\left[t_{l}-t_{l+1},+\infty\right]=t_{l}-t_{l+1}, \theta[0,+\infty]=0\right)}{\mathbb{P}(\theta[0,+\infty]=0)} \\
=\frac{\mathbb{P}\left(\theta\left[t_{l}-t_{l+1},-1\right]=t_{l}-t_{l+1}, \theta[0,+\infty]=0\right)}{\mathbb{P}(\theta[0,+\infty]=0)} \\
=\frac{\mathbb{P}\left(\theta\left[t_{l}-t_{l+1},-1\right]=t_{l}-t_{l+1}\right) \mathbb{P}(\theta[0,+\infty]=0)}{\mathbb{P}(\theta[0,+\infty]=0)} \\
=\mathbb{P}\left(\theta\left[t_{l}-t_{l+1},-1\right]=t_{l}-t_{l+1}\right) .
\end{gathered}
$$

De um outro lado, como

$$
\mathbb{P}\left(\theta\left[t_{l}-t_{l+1},+\infty\right]=t_{l}-t_{l+1} \mid \theta[0,+\infty]=0\right)=\mathbb{P}\left(\xi_{t_{l}-t_{l+1}}=1 \mid \xi_{0}=1\right)
$$

re-escrevendo (2.11) utilizando a cadeia $\boldsymbol{\xi}$ nos dá a relação que queríamos. Acrescentando o fato que para todo $n, \mathbb{P}(\theta[n,+\infty]=n)>0$, concluimos que a cadeia $\boldsymbol{\xi}$ é uma cadeia de renovação. Segue que os tempos $n$ tais que $T_{n}=1$ dividem a cadeia $\mathbf{X}(\mathbf{U})$ em blocos i.i.d. Isso porque por construção, a cadeia $\mathbf{X}(\mathbf{U})$ é uma concatenação i.i.d. (utilizamos segmentos disjuntos de $\mathbf{U}$, que é uma sequência i.i.d.) de blocos obtidos utilizando o algoritmo. 
No nosso caso, podemos mostrar que nas condições nas quais podemos realizar uma simulação perfeita, a cadeia obtida é regenerativa, e o tamanho médio dos blocos independentes (isto é entre dois 1's consecutivos na sequência T) é finita (ver Teorema 5.3 e a prova na Seção 7). No entanto, não sabemos se uma simulação perfeita implica a existência de um esquema regenerativo em geral. Em Comets et al. (2002), os autores obtiveram um regime no qual conseguem fazer uma simulação perfeita quando $\mathbb{P}(\theta[0,+\infty]=0)=0$, isto é, uma situação na qual pode não ter esquema regenerativo.

Uma outra questão interessante é: será que se uma cadeia estocástica estacionária tem um esquema regenerativo, então existe uma maneira de fazer uma simulação perfeita desta cadeia, utilizando um acoplamento desde o passado? Não sabemos responder a esta pergunta. Mas temos exemplos (ver Seção 4) de cadeias regenerativas para as quais não sabemos fazer simulação perfeita.

Para resumir, não sabemos se uma destas implicações é verdadeira

$$
\begin{aligned}
& \text { esquema regenerativo } \Rightarrow \text { simulação perfeita ?? } \\
& \text { esquema regenerativo } \Leftarrow \text { simulação perfeita ?? }
\end{aligned}
$$

\subsection{Aproximação Markoviana}

Nesta seção, introduzimos a noção de aproximação Markoviana de uma cadeia de alcance infinito. A questão intuitiva é: quão precisamente podemos aproximar uma cadeia (que podemos simular com o algoritmo acima) com cadeias de Markov?

Para formalizar esta questão, utilizamos dois conceitos: a distância de Ornstein e a aproximação canônica markoviana de uma cadeia. As definições são dadas a seguir. Seja $(\tau, p)$ uma árvore probabilística qualquer. Definimos a árvore $\left.\tau\right|_{k}$ por

$$
\left.\tau\right|_{k}=\{v \in \tau:|v| \leq k\} \cup\left\{v_{-k}^{-1}: v \in \tau,|v|>k\right\}
$$

é a árvore truncada na altura $k$.

\section{Exemplos}

- Se $\tau$ é a árvore da Figura 2.3, então $\left.\tau\right|_{4}$ é a árvore da Figura 2.5. 
- Se $\tau$ é a árvore da Figura 5.2, então $\left.\tau\right|_{5}$ é a árvore da Figura 2.6.

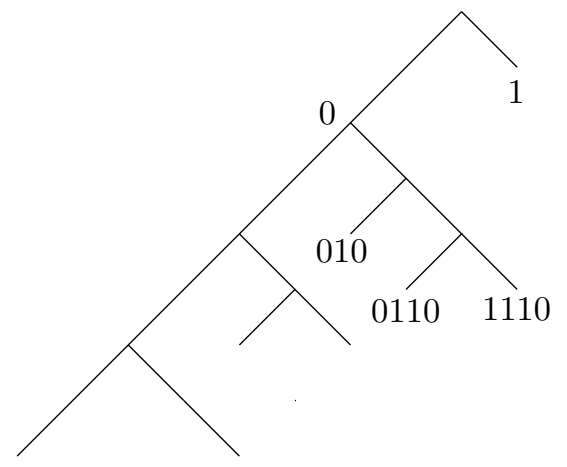

Figura 2.5: Árvore da Figura 2.3 truncada na altura 4.

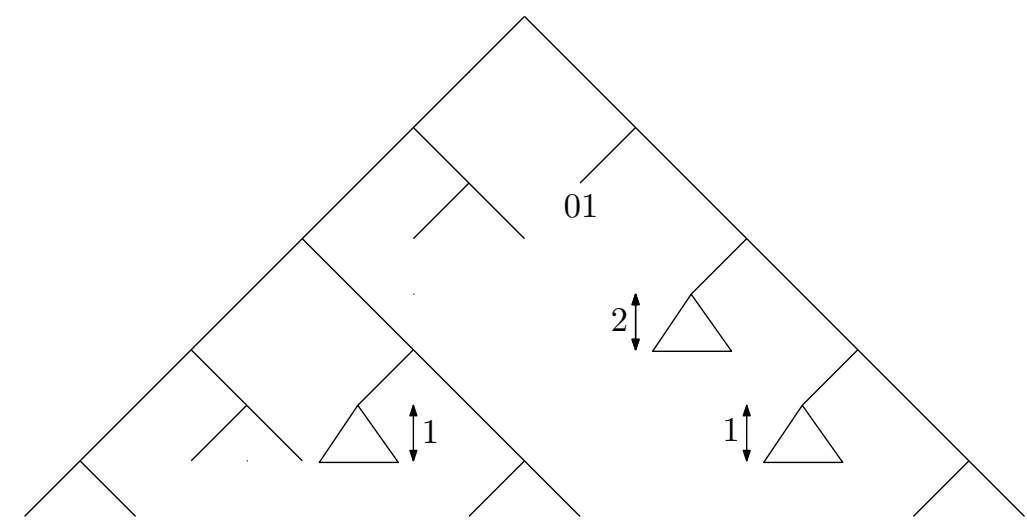

Figura 2.6: Árvore da Figura 5.2 truncada na altura 5. Os pequenos triângulos representam os triângulos da árvore inicial truncados na altura indicada.

Consideramos agora a sequência de árvores probabilísticas $\left\{\left(\tau^{[k]}, p^{[k]}\right)\right\}_{k \geq 0}$ definidas por

$$
\tau^{[k]}=\left.\tau\right|_{k}
$$

e para todo $a \in A$ e $v \in \tau^{[k]}$

$$
p^{[k]}(a \mid v)=\mathbb{P}\left(X_{0}=a \mid X_{-|v|}^{-1}=v\right)
$$

onde $\mathbf{X}$ denota uma cadeia estacionária compatível com $(\tau, p)$. 
Definição 2.15 (Aproximação Markoviana canónica). A cadeia $\mathbf{X}^{[k]}$ compatível com $\left(\tau^{[k]}, p^{[k]}\right)$ é chamada aproximação Markoviana canónica de $\mathbf{X}$.

Definição 2.16 (Distância de Ornstein). A distância $\bar{d}$ de Ornstein entre duas cadeias $\mathbf{X}$ e $\mathbf{Y} e ́$ definida por

$$
\bar{d}(\mathbf{X}, \mathbf{Y})=\inf \left\{\mathbb{P}\left(\tilde{X}_{0} \neq \tilde{Y}_{0}\right):(\tilde{\mathbf{X}}, \tilde{\mathbf{Y}}) \text { acoplamentos estacionários de } \mathbf{X} \text { e } \mathbf{Y}\right\}
$$

A questão feita acima pode ser reformulada da seguinte maneira: sob que condições uma cadeia $\mathbf{X}$ de alcance variável não limitado é o limite (na distância $\bar{d}$ ) da sua aproximação Markoviana canónica? Isto é

$$
\bar{d}\left(\mathbf{X}, \mathbf{X}^{[k]}\right) \stackrel{k \rightarrow+\infty}{\longrightarrow} 0 ?
$$

Na Seção 5.3, o Teorema 5.4 diz que nas condições nas quais sabemos fazer uma simulação perfeita da cadeia, ela também verifica a convergência acima. 


\section{Capítulo 3}

\section{Árvores não limitadas: resultados introdutórios}

Nas três primeiras seções deste capítulo mostramos exemplos de árvores probabilísticas não limitadas para as quais obtemos comportamentos patológicos. Primeiro, apresentamos um exemplo em que não existe cadeia estacionária compatível, em seguida mostramos um exemplo em que existe uma única cadeia estacionária compatível, mas ela visita os contextos de tamanho infinito com probabilidade 1 e por último, um caso em que existem duas cadeias estacionárias compatíveis com a árvore. Na quarta seção, damos critérios suficientes sobre a forma da árvore probabilística para garantir que quando existe alguma cadeia estacionária compatível, ela visita os contextos de tamanho infinito com probabilidade zero. Os resultados desta quarta seção são originais.

\subsection{Um exemplo de não existência}

Considere a árvore esparsa com as probabilidades de transição $p\left(1 \mid 0^{i} 1\right)=p_{i}$ verificando

$$
\sum_{i \geq 0} p_{i}<\infty \quad \text { e } \quad p_{\infty}=1
$$

Não existe medida invariante para esta árvore. Para ver isto, vamos dividir o conjunto $A^{\mathbb{Z}}$ da seguinte maneira

$$
A^{\mathbb{Z}}=\mathbf{0} \cup\left(A^{\mathbb{Z}} \backslash \mathbf{0}\right)
$$

Vamos mostrar que nem $\mathbf{0}$ nem $A^{\mathbb{Z}} \backslash \mathbf{0}$ podem ser suporte da medida invariante.

- Suponhamos que a medida invariante $\mathbb{Q}$ tem suporte sobre 0. Temos $\mathbb{Q}\left(X_{0}=1 \mid X_{-\infty}^{-1}=\right.$ $\left.0_{-\infty}^{-1}\right)=1$ o que significa que $\mathbf{0}$ não pode ser suporte da medida invariante.

- Suponhamos agora que a medida invariante $\mathbb{Q}$ tem suporte sobre $A^{\mathbb{Z}} \backslash \mathbf{0}$. Pegamos um passado 
$x_{-\infty}^{-1}$ tal que $x_{-i}=1$ para algum $i \geq 1$. Vamos ver que com probabilidade positiva vai aparecer um bloco infinito de zeros na realização de $\left(X_{n}\right)_{n \geq 0}$ : supondo que o último 1 da sequência $x_{-\infty}^{-1}$ tenha aparecido no tempo $-i$, temos

$$
\mathbb{Q}\left(\cap_{n \geq 0}\left\{X_{n}=0\right\} \mid x_{-i}^{-1}=0^{i-1} 1\right)=\prod_{j \geq i-1}\left(1-p_{j}\right) \geq \prod_{j \geq 0}\left(1-p_{j}\right)>0
$$

devido à somabilidade dos $p_{i}$ 's. Portanto, $A^{\mathbb{Z}} \backslash \mathbf{0}$ não pode ser o suporte da medida invariante.

Isso implica que não existe nenhuma medida invariante compatível com esta árvore probabilística. A razão desta patologia parece ser dupla. Primeiro, a discontinuidade da árvore probabilística:

$$
\beta_{i}=\sup _{a \in A} \sup \left\{\left|p\left(a \mid x_{-\infty}^{-1}\right)-p\left(a \mid y_{-\infty}^{-1}\right)\right|: x_{-\infty}^{-1} \stackrel{i}{=} y_{-\infty}^{-1}\right\}=\left|p_{i}-p_{\infty}\right| \stackrel{i \rightarrow+\infty}{\longrightarrow} p_{\infty}=1
$$

Mas a discontinuidade não é suficiente para garantir a não existência da medida invariante (é justamente o assunto desta tese). Também é importante o fato que tenha um peso positivo sobre o ponto $\mathbf{0}$, onde justamente existe uma discontinuidade.

\subsection{Probabilidade positiva de ter um contexto de tamanho infinito}

Dizemos que a cadeia estacionária X compatível com $(\tau, p)$ "visita" os contextos de tamanho infinito com probabilidade positiva se

$$
\mathbb{P}\left(\left|c_{\tau}\left(X_{-\infty}^{-1}\right)\right|=+\infty\right)>0
$$

Considerando de novo a árvore esparsa $\operatorname{com} \sum_{i \geq 0} p_{i}<\infty$, mas desta vez com $p_{\infty}=0$ podemos ver que $(\tau, p)$ é contínua. Portanto a cadeia estacionária existe e é única, pois a taxa de continuidade converge para zero e é somável. A mesma conta que no parágrafo precedente mostra que começando com qualquer sequência, a cadeia vai acabar com uma sequência infinita de zeros, com probabilidade positiva. Mas desta vez a sequência infinita de zeros é invariante pois $p_{\infty}=0$. Portanto a única cadeia estacionária compatível com a árvore probabilística é a sequência "todo zero", que é justamente o único contexto de tamanho infinito da árvore. Este exemplo é muito patológico, pois desaparece um símbolo do alfabeto (o símbolo 1) com probabilidade positiva ao 
longo da realização. Podemos evitar este tipo de situação impondo por exemplo que o símbolo 1 seja $\epsilon$-regular para algum $\epsilon>0$. Neste caso, é claro que aparecem uma infinidade de 1's, e a medida estacionária tem suporte em $A^{\mathbb{Z}} \backslash \mathbf{0}$ (isso é verdade mesmo com condições mais fracas como veremos na Seção 4.1). No entanto, a condição de regularidade não é suficiente para garantir que a cadeia estacionária não verifique (3.1). Basta considerar a árvore totalmente ramificada sobre $A$ finito, e com $\epsilon$-regularidade. É claro que neste caso, a cadeia (se existir), vai visitar os contextos de tamanho infinito, já que não existem contextos de tamanho finito.

\subsection{Exemplo em que a cadeia não é irredutível}

Considerando de novo a árvore esparsa com $\lim _{i \rightarrow+\infty} p_{i}=0, p_{\infty}=0$ e $\sum_{i \geq 0} p_{i}=+\infty$, podemos ver que $(\tau, p)$ é contínua. Portanto, existe alguma cadeia estacionária, chamaremos $\mathbb{Q}$ sua lei (ao longo desta tese, usaremos sempre $\mathbb{Q}$ para denotar a lei invariante compatível com a árvore esparsa). Se começamos com um passado $0_{-\infty}^{-1}$, podemos ver que continuaremos a botar 0 's até o infinito no futuro. Isto é, a cadeia todo 0 é estacionária. Mas também, se começamos com um passado em $A^{-\mathbb{N}} \backslash 0^{-\mathbb{N}}$, a condição $\sum_{i \geq 0} p_{i}=+\infty$ faz com que a cadeia resultante terá uma infinidade de 1's (esta conta foi feita na tese de doutorado de Esteves (2007)).

Este não é um caso de transição de fase, porque a lei estacionária $P^{0}$ (aquela que dá peso 1 á sequência todo zero) não verifica:

$$
\mathbb{P}^{0}\left(X_{1}^{l}=a_{1}^{l}\right)>0, \forall a_{1}^{l} \in A^{l} .
$$

Portanto, dizemos que se trata de um caso de irredutibilidade.

\subsection{Probabilidade zero de ter um contexto de tamanho infinito}

Nesta parte, temporariamente, consideramos árvores probabilísticas $(\tau, p)$ contínuas, sobre alfabeto $A$ finito, e com condição de $\epsilon$-regularidade para todos os elementos de $A$. Nestas condições, a existência de ao menos uma cadeia estacionária compatível com $(\tau, p)$ é garantida. A nossa meta aqui é achar critérios sobre a forma de $\tau$ para que qualquer cadeia estacionária $\mathbf{X}$ (de lei $\mathbb{P}$ ) compatível com $(\tau, p)$ verifique

$$
\mathbb{P}\left(\left|c_{\tau}\left(X_{-\infty}^{-1}\right)\right|<+\infty\right)=1
$$


As condições de continuidade e de regularidade podem parecer restritivas, mas é o preço a pagar para obter critérios bastante gerais sobre a forma da árvore. Os capítulos 4 e 5 trazem um outro tipo de critério, no qual não se precisa dessas restrições, mas fazendo hipóteses mais finas sobre a estrutura da árvore. Uma observação importante é o fato que não sabemos se os critérios que seguem implicam a unicidade da cadeia estacionária compatível com $(\tau, p)$. Começamos por um lema útil para o resto da seção.

Lema 3.1. Seja uma árvore probabilística $(\tau, p)$ contínua sobre um alfabeto enumerável $A$. Qualquer cadeia estacionária $\mathbf{X}$ (de lei $\mathbb{P})$ compatível com $(\tau, p)$ verifica

$$
\left(\min _{i} \inf _{v \in \tau} p\left(a_{i} \mid v\right)\right)^{n} \leq \mathbb{P}\left(X_{0}^{n-1}=a_{0}^{n-1}\right) \leq\left(\max _{i} \sup _{v \in \tau} p\left(a_{i} \mid v\right)\right)^{n}, \forall a_{0}^{n-1} \in A^{n}
$$

Demonstração. Primeiro utilizamos a decomposição

$$
\begin{gathered}
\mathbb{P}\left(a_{0}^{n-1} \mid a_{-\infty}^{-1}\right)=\mathbb{P}\left(a_{n-1} \mid a_{0}^{n-2} a_{-\infty}^{-1}\right) \mathbb{P}\left(a_{n-2} \mid a_{0}^{n-3} a_{-\infty}^{-1}\right) \ldots \mathbb{P}\left(a_{0} \mid a_{-\infty}^{-1}\right) \\
\quad=p\left(a_{n-1} \mid c_{\tau}\left(a_{0}^{n-2} a_{-\infty}^{-1}\right)\right) p\left(a_{n-2} \mid c_{\tau}\left(a_{0}^{n-3} a_{-\infty}^{-1}\right)\right) \ldots p\left(a_{0} \mid c_{\tau}\left(a_{-\infty}^{-1}\right)\right)
\end{gathered}
$$

portanto para todo $n \geq 1$ e $a_{0}^{n-1} \in A^{n}$

$$
\left(\min _{i} \inf _{v \in \tau} p\left(a_{i} \mid v\right)\right)^{n} \leq \mathbb{P}\left(X_{0}^{n-1}=a_{0}^{n-1} \mid X_{-\infty}^{-1}=a_{-\infty}^{-1}\right) \leq\left(\max _{i} \sup _{v \in \tau} p\left(a_{i} \mid v\right)\right)^{n} .
$$

Por outro lado, temos

$$
\mathbb{P}\left(X_{0}^{n-1}=x_{0}^{n-1}\right)=\int_{A^{\mathbb{Z}}} \mathbb{P}\left(X_{0}^{n-1}=a_{0}^{n-1} \mid X_{-\infty}^{-1}=a_{-\infty}^{-1}\right) d \mathbb{P}(\mathbf{a}),
$$

$\log 0$

$$
\left(\min _{i} \inf _{v \in \tau} p\left(a_{i} \mid v\right)\right)^{n} \int_{A^{\mathbb{Z}}} d \mathbb{P}(\mathbf{a}) \leq \mathbb{P}\left(X_{0}^{n-1}=a_{0}^{n-1}\right) \leq\left(\max _{i} \sup _{v \in \tau} p\left(a_{i} \mid v\right)\right)^{n} \int_{A^{\mathbb{Z}}} d \mathbb{P}(\mathbf{a}) .
$$

Proposição 3.1. Seja $(\tau, p)$ uma árvore probabilística contínua sobre um alfabeto finito A. Se 
todos os elementos de A são $\epsilon$-regulares e $\tau$ possui um número enumerável de contextos de tamanho infinito, então (3.2) vale para qualquer cadeia estacionária $\mathbf{X}$ compativel com $(\tau, p)$.

Demonstração. Vamos enumerar os contextos infinitos de $\tau$ por $\left\{v^{i}\right\}_{i \in\{1,2, \ldots, N\}}$ onde $N$ é o número de contextos de tamanho infinito, possivelmente infinito $(N \leq+\infty)$. Podemos escrever

$$
\mathbb{P}\left(\left|c_{\tau}\left(X_{-\infty}^{-1}\right)\right|=+\infty\right)=\sum_{i=1}^{N} \mathbb{P}\left(c_{\tau}\left(X_{-\infty}^{-1}\right)=v^{i}\right)
$$

devido ao fato que $\tau$ forma uma partição de $A^{-\mathbb{N}}$. É fácil ver que se todos os elementos de $A$ são $\epsilon$-regulares, então temos $\epsilon \leq p(a \mid v) \leq 1-\epsilon$ para todo $a$ em $A$ e todo $v \in \tau$. Nesse caso temos pelo Lema 3.1

$$
\epsilon^{n} \leq \mathbb{P}\left(X_{0}^{n-1}=a_{0}^{n-1}\right) \leq(1-\epsilon)^{n}, \quad \forall a_{0}^{n-1} \in A^{n}
$$

Portanto $\mathbb{P}\left(c_{\tau}\left(X_{-\infty}^{-1}\right)=v^{i}\right)=0$ para todo $i$, logo

$$
\mathbb{P}\left(\left|c_{\tau}\left(X_{-\infty}^{-1}\right)\right|=+\infty\right)=0
$$

Definição 3.2. Dado uma árvore de contextos $\tau$, definimos $\tau^{k, \infty}$ por

$$
\tau^{k, \infty}=\left\{v_{-k}^{-1}: v \in \tau,|v|=+\infty\right\}
$$

Proposição 3.3. Seja $(\tau, p)$ uma árvore probabilística contínua sobre um alfabeto finito A. Se todos os elementos de A são $\epsilon$-regulares e $\tau$ é tal que

$$
\limsup _{k \rightarrow \infty} \frac{\log \left|\tau^{k, \infty}\right|}{C_{\epsilon} k}<1
$$

com

$$
C_{\epsilon}=\frac{1}{1-\epsilon}
$$

então (3.2) vale para qualquer cadeia estacionária $\mathbf{X}$ compatível com $(\tau, p)$. 
Demonstração. Temos

$$
\begin{aligned}
& \mathbb{P}\left(\left|c_{\tau}\left(X_{-\infty}^{-1}\right)\right|=\infty\right)=\mathbb{P}\left(\cap_{i \geq 1}\left\{X_{-i}^{-1} \in \tau^{i, \infty}\right\}\right)=\lim _{k \rightarrow \infty} \mathbb{P}\left(\cap_{i=1}^{k}\left\{X_{-i}^{-1} \in \tau^{i, \infty}\right\}\right) \\
= & \lim _{k \rightarrow \infty} \mathbb{P}\left(X_{-k}^{-1} \in \tau^{k, \infty}\right)=\lim _{k \rightarrow \infty} \sum_{a_{-k}^{-1} \in \tau^{k, \infty}} \mathbb{P}\left(X_{-k}^{-1}=a_{-k}^{-1}\right) \leq \lim _{k \rightarrow \infty}\left|\tau^{k, \infty}\right|(1-\epsilon)^{k},
\end{aligned}
$$

onde a última desigualdade é devido ao fato que todos os elementos de $A$ são $\epsilon$-regulares e utilizando o Lema 3.1. Nas condições da proposição existe um número real $\alpha>0$ tal que para todo $k$ suficientemente grande

$$
\left|\tau^{k, \infty}\right| \leq\left(\frac{1}{1-\epsilon}\right)^{k(1-\alpha)}
$$

portanto

$$
\mathbb{P}\left(\left|c_{\tau}\left(X_{-\infty}^{-1}\right)\right|=\infty\right) \leq \lim _{k \rightarrow \infty}(1-\epsilon)^{\alpha k}=0
$$

Nas próximas seções veremos outros critérios que garantem (3.2). São critérios sobre a forma da árvore e as probabilidades de transição que não exigem a condição de continuidade. 


\section{Capítulo 4}

\section{Árvores tendo um contexto terminal}

As árvores que possuem um contexto (de tamanho finito) que aparece como prefixo de todos os contextos nos quais ele aparece são denominadas árvores com contexto terminal. Chamaremos este contexto $w$. Para qualquer $v \in \tau \operatorname{com}|v|>|w|$, temos duas possibilidades: (i) $w$ não é uma sub-sequência de $v$ (escrevemos neste caso $w \notin v$ ), (ii) ou $v_{-|v|}^{-|v|+|w|-1}=w$. Denotamos por $\tau_{w}$ o subconjunto de $\tau$ formado pelos contextos nos quais aparece $w$. Um caso particular é a árvore esparsa definida por (2.2) que tem como contexto terminal o símbolo 1 , nesse caso $\mathcal{W}=\{1\}$ e $\tau_{w}=\left\{10^{i}\right\}_{i \geq 0}$. Outros exemplos estão dados nas Figuras 4.1 e 4.2.

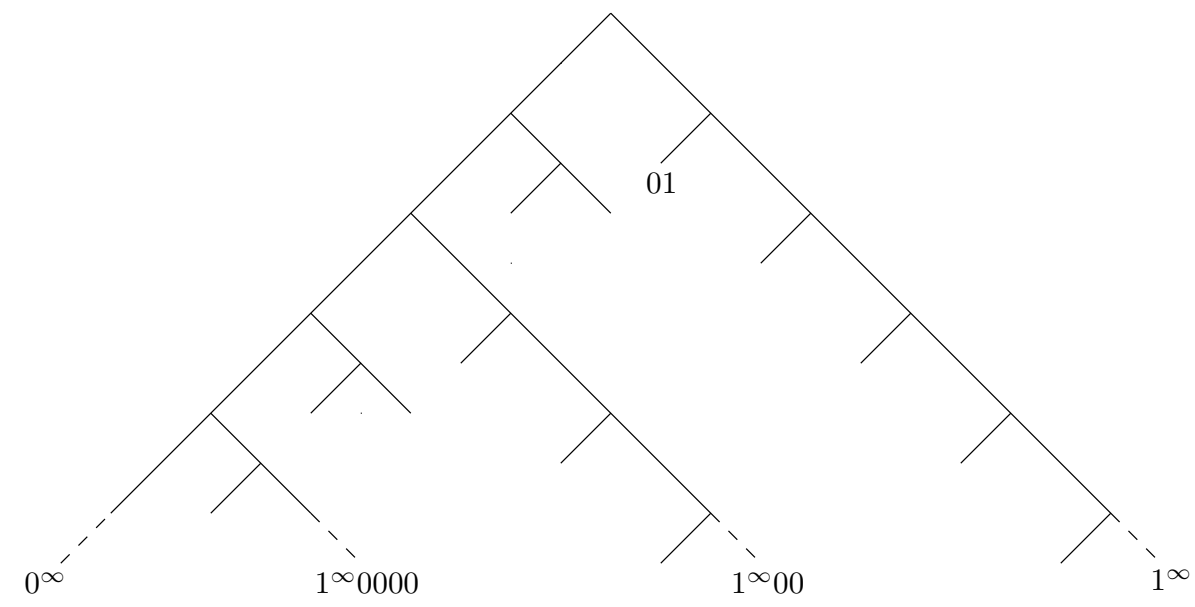

Figura 4.1: Árvore sobre $A=\{0,1\}$ tendo 01 como contexto terminal.

Seja uma árvore de contextos não limitada $\tau$ e $w$ um dos seus contextos. Para qualquer contexto $v$ definimos uma função $m^{w}: A^{*} \rightarrow \mathbb{N}$ por

$$
m^{w}(v)=\inf \left\{j: 0 \leq j \leq|v|-|w|, \text { tal que } v_{-j-|w|}^{-j-1}=w\right\}
$$




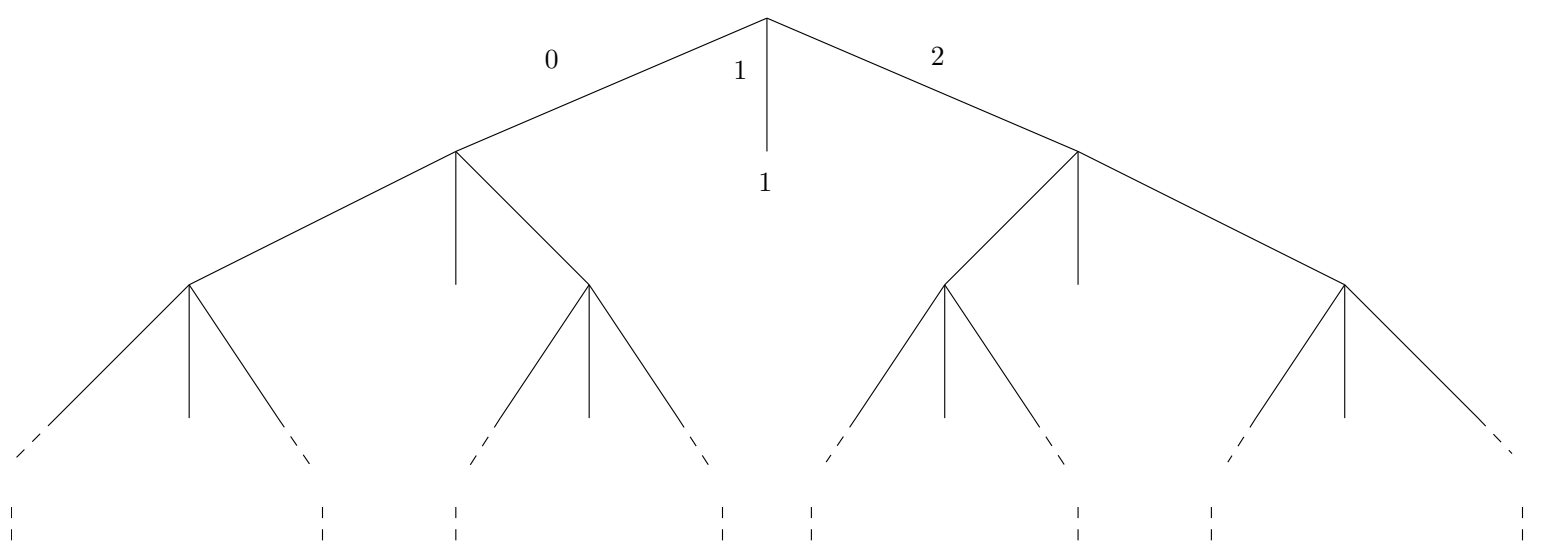

Figura 4.2: Árvore sobre $A=\{0,1,2\}$ tendo o símbolo 1 como contexto terminal.

com a convenção que $m^{w}(v)=+\infty$ se o conjunto de índices é vazio. Com esta definição, se o contexto $v$ é tal que $m^{w}(v)=k$, então podemos escrevê-lo da seguinte maneira

$$
v=\left(v_{-|v|}, \ldots, v_{-k-|w|-1}\right) w\left(v_{-k}, \ldots, v_{-1}\right)
$$

onde $w \notin v_{-k}^{-1}$. Se $w$ é um contexto terminal de $\tau$, então

$$
v=w\left(v_{-k}, \ldots, v_{-1}\right) .
$$

Denotamos por $\mathcal{I}_{k}^{w}$ o conjunto de sequências introdutórias $\left(v_{-k}, \ldots, v_{-1}\right)$ correspondendo aos contextos $v \in \tau$ tal que $m^{w}(v)=k$ :

$$
\mathcal{I}_{k}^{w}=\left\{\left(v_{-k}, \ldots, v_{-1}\right) \in A^{k}: v \in \tau \quad \text { e } \quad m^{w}(v)=k\right\}
$$

Começamos este capítulo considerando a árvore esparsa. Vários cálculos explícitos são possíveis neste caso. Em particular sob condições fracas sobre as probabilidades de transição, podemos mostrar que possui uma única medida estacionária compatível (Seção 4.1), que esta medida é também reversível (Seção 4.3), e podemos fazer exata a conta da entropia em função das probabilidades de transição (Seção 4.4). Vários cálculos valem também para alfabeto qualquer quando o contexto terminal $w$ tem tamanho 1 , isto é, pertence ao alfabeto $A$.

Terminamos este capítulo mostrando como podemos realizar uma simulação perfeita da única cadeia estacionária compatível com uma árvore tendo um contexto terminal e $\epsilon$-regular. 


\subsection{Existência e unicidade da medida invariante}

Definimos

$$
p_{i}(b)=\inf \left\{p_{i}(b \mid s b): s \in \mathcal{I}_{i}^{b}\right\} .
$$

Proposição 4.1. Seja $(\tau, p)$ uma árvore de contextos probabilística tendo como contexto terminal um elemento $b$ do alfabeto. Se

$$
\sum_{i \geq 1} \prod_{j=0}^{i-1}\left(1-p_{j}(b)\right)<+\infty
$$

então existe uma única cadeia estacionária compatível com $(\tau, p)$. Esta cadeia verifica (3.2).

Caso $A$ binário $\{0,1\}$. Este é o caso da árvore esparsa, $b=1$. Esta cadeia é a cadeia de renovação em tempo discreto. Podemos ver esta cadeia como uma concatenação i.i.d. de blocos $0^{i-1} 1$ (também chamados ciclos $C_{i}$ ), com a lei $Q$ sobre $\{1,2, \ldots\}$ (tamanho do bloco):

$$
Q(1)=p(1 \mid 1)=p_{0}
$$

$\mathrm{e}$

$$
Q(i+1):=Q\left(0^{i} 1\right)=p(0 \mid 1) p(0 \mid 01) \ldots p\left(0 \mid 0^{i-1} 1\right) p\left(1 \mid 0^{i} 1\right)=p_{i} \prod_{j=0}^{i-1}\left(1-p_{j}\right), \quad \forall i \geq 1
$$

Sabe-se que a existência de uma versão estacionária desta cadeia é garantida se a esperança do tamanho dos blocos escolhidos com a lei $Q$ é finita (ver por exemplo Thorisson (2000)). Vamos verificar que isso é o caso nas condições da proposição. Calculamos

$$
\sum_{i \geq 0}(i+1) Q(i+1)=p_{0}+\sum_{i \geq 1}(i+1) p_{i} \prod_{j=0}^{i-1}\left(1-p_{j}\right)
$$

e observando que para $i \geq 1$

$$
p_{i} \prod_{k=0}^{i-1}\left(1-p_{k}\right)=\left(1-1+p_{i}\right) \prod_{k=0}^{i-1}\left(1-p_{k}\right)=\prod_{k=0}^{i-1}\left(1-p_{k}\right)-\prod_{k=0}^{i}\left(1-p_{k}\right)
$$

podemos ver que o somatório em $i$ pode ser desenvolvido como somas telescópicas na equação (4.2) 
(tomando $\left.p_{0}=1-\left(1-p_{0}\right)\right)$ :

$$
\sum_{i \geq 0}(i+1) Q(i+1)=1+\sum_{i \geq 1} \prod_{j=0}^{i-1}\left(1-p_{j}\right)
$$

Nas condições da proposição (desigualdade (4.1)), este último somatório é finito. Isso prova a existência da cadeia estacionária.

A prova é um pouco mais elaborada no caso $A$ enumerável, mas não é mais difícil.

Caso A enumerável. Neste caso, não se trata de uma cadeia de renovação porque os ciclos não são inteiramente determinados pelo tamanho, no entanto, continua sendo uma concatenação i.i.d. de ciclos do tipo $a_{0}^{j-1} b, a_{0}^{j-1} \in(A \backslash\{b\})^{j}$, com lei $Q$ :

$$
Q\left(a_{0}^{j-1} b\right)=p\left(a_{0} \mid b\right) p\left(a_{1} \mid a_{0} b\right) \ldots p\left(a_{j-1} \mid a_{0}^{j-2} b\right) p\left(b \mid a_{0}^{j-1} b\right) .
$$

Utilizamos a notação $A \backslash\{b\}=A_{\bar{b}}$. Neste caso, também basta verificar que a esperança do tamanho dos ciclos escolhidos por $Q$ é finita. A probabilidade que seja escolhido um ciclo de tamanho $j+1$, $j \geq 1$ é

$$
\sum_{a_{0}^{j-1} \in A_{\bar{b}}^{j}} Q\left(a_{0}^{j-1} b\right)
$$

A probabilidade de escolher um ciclo de tamanho 1 é $p(b \mid b)$. Portanto, temos que calcular a esperança do tamanho, que é dada por

$$
p(b \mid b)+\sum_{j \geq 1}(j+1) \sum_{a_{0}^{j-1} \in A_{\bar{b}}^{j}} Q\left(a_{0}^{j-1} b\right) .
$$

Observamos primeiro que para $j \geq 1$

$$
p\left(a_{0} \mid b\right) p\left(a_{1} \mid a_{0} b\right) \ldots p\left(a_{j-1} \mid a_{0}^{j-2} b\right) p\left(b \mid a_{0}^{j-1} b\right)=p\left(a_{0} \mid b\right) p\left(a_{1} \mid a_{0} b\right) \ldots p\left(a_{j-1} \mid a_{0}^{j-2} b\right)\left(1-\sum_{a_{j} \in A_{\bar{b}}} p\left(a_{j} \mid a_{0}^{j-1} b\right)\right)
$$


onde $a_{0}^{-1}=\emptyset$. Portanto

$$
\begin{gathered}
\sum_{a_{0}^{j-1} \in A_{\bar{b}}^{j}} Q\left(a_{0}^{j-1} b\right)=\sum_{a_{0}^{j-1} \in A_{\bar{b}}^{j}} p\left(a_{0} \mid b\right) p\left(a_{1} \mid a_{0} b\right) \ldots p\left(a_{j-1} \mid a_{0}^{j-2} b\right)-\sum_{a_{0}^{j} \in A_{\bar{b}}^{j+1}} p\left(a_{0} \mid b\right) p\left(a_{1} \mid a_{0} b\right) \ldots p\left(a_{j} \mid a_{0}^{j-1} b\right) \\
=: B_{j}-B_{j+1},
\end{gathered}
$$

e também, $p(b \mid b)=1-\sum_{a_{0} \in A_{\bar{b}}} p\left(a_{0} \mid b\right)=: 1-B_{1}$. Portanto, $p(b \mid b)+\sum_{j \geq 1}(j+1) \sum_{a_{0}^{j-1} \in A_{\bar{b}}^{j}} Q\left(a_{0}^{j-1} b\right)$ é de novo um tipo de somátorio telescópico:

$$
1-B_{1}+2\left(B_{1}-B_{2}\right)+3\left(B_{2}-B_{3}\right)+\ldots=1+\sum_{j \geq 1} B_{j}
$$

Agora, vamos estudar $B_{j}, j \geq 1$ : como $\sum_{a \in A_{\bar{b}}} p(a \mid v b)=1-p(b \mid v b) \leq 1-p_{|v|}(b)$, temos

$$
\begin{gathered}
\sum_{a_{0}^{j-1} \in A_{\bar{b}}^{j}} p\left(a_{0} \mid b\right) p\left(a_{1} \mid a_{0} b\right) \ldots p\left(a_{j-1} \mid a_{0}^{j-2} b\right) \leq \sum_{a_{0}^{j-2} \in A_{\bar{b}}^{j-1}} p\left(a_{0} \mid b\right) p\left(a_{1} \mid a_{0} b\right) \ldots p\left(a_{j-2} \mid a_{0}^{j-1} b\right)\left(1-p_{j-1}(b)\right) \\
\leq \sum_{a_{0}^{j-3} \in A_{\bar{b}}^{j-2}} p\left(a_{0} \mid b\right) p\left(a_{1} \mid a_{0} b\right) \ldots p\left(a_{j-3} \mid a_{0}^{j-2} b\right)\left(1-p_{j-2}(b)\right)\left(1-p_{j-1}(b)\right) \\
\leq \ldots \leq \prod_{i=0}^{j-1}\left(1-p_{i}(b)\right) .
\end{gathered}
$$

Logo

$$
\sum_{j \geq 0}(j+1) \sum_{a_{0}^{j-1} \in A_{\bar{b}}^{j}} Q\left(a_{0}^{j-1} b\right) \leq 1+\sum_{j \geq 1} \prod_{i=0}^{j-1}\left(1-p_{i}(b)\right)
$$

Esta útltima quantidade é finita nas condições da Proposição, portanto a esperança do tamanho dos ciclos escolhidos com $Q$ é finita.

A prova da unicidade segue simplesmente do fato que a cadeia é regenerativa. Como mencionamos, sempre podemos pensar esta cadeia como sendo uma concatenação i.i.d. de ciclos $C_{i}, i \geq 0$ com lei $Q$. A necessidade de se ter uma esperança finita do tamanho dos ciclos vem do fato que neste caso, podemos definir uma versão estacionária da cadeia escolhendo o tamanho do primeiro ciclo $C_{0}$ com a distribuição

$$
c_{k}=\frac{\sum_{i \geq k+1} Q(i)}{\mu}, \quad k \geq 0
$$


onde $\mu$ denota a esperança do tamanho dos ciclos sob $Q: \mu:=\sum_{i \geq 1} i Q(i)$. Escolhendo os ciclos subsequentes $C_{i}, i \geq 1$ de acordo com a lei $Q$, a cadeia que obtemos é estacionária. Este fato pode ser encontrado em Lindvall (1992) ou Thorisson (2000).

\subsection{Cálculo explícito da medida invariante}

Já sabemos que existe uma única cadeia estacionária $\mathbf{X}$ (de lei $\mathbb{Q}$ ) compatível com a árvore esparsa quando as probabilidades de transição verificam (4.1). Além disso, podemos fazer o cálculo explícito da probabilidade estacionária de qualquer cilindro.

Proposição 4.2. Nas mesmas condições que na Proposição 4.1 temos para qualquer cilindro $a_{0}^{l-1} \in$ $A^{l}$

$$
\mathbb{Q}\left(a_{0}^{l-1}\right)=\frac{\sum_{i \geq 1} \sum_{a_{-i+1}^{-1} \in A_{\bar{b}}^{i-1}} \prod_{j=-i+1}^{l-1} p\left(a_{j} \mid a_{-i+1}^{j-1} b\right)}{1+\sum_{a \in A_{\bar{b}}} \sum_{i \geq 1} \sum_{a_{-i+1}^{-1} \in A_{\bar{b}}^{i-1}} \prod_{j=-i+1}^{l-1} p\left(a_{j} \mid a_{-i+1}^{j-1} b\right)} .
$$

No caso particular da cadeia esparsa, obtemos para $a_{0}^{l-1} \in\{0,1\}^{l}$

$$
\mathbb{Q}\left(a_{0}^{l-1}\right)=\frac{\sum_{i \geq 1} \prod_{j=0}^{i-2}\left(1-p_{j}\right) \prod_{j=0}^{l-1} p\left(a_{j} \mid a_{0}^{j-1} 0^{i-1} 1\right)}{\left(1+\sum_{i \geq 1} \prod_{j=0}^{i-1}\left(1-p_{j}\right)\right)} .
$$

Caso A binário. Definimos

$$
\theta[0]:=\max \left\{i \leq 0: X_{i}=1\right\}
$$

Sabemos que

$$
\mathbb{Q}(\theta[0]>-\infty)=1
$$

portanto podemos realizar a seguinte partição para todo $a_{0}^{l-1} \in\{0,1\}^{l}$ :

$$
\mathbb{Q}\left(X_{0}^{l-1}=a_{0}^{l-1}\right)=\sum_{i \geq 1} \mathbb{Q}\left(X_{0}^{l-1}=a_{0}^{l-1}, \theta[0]=-i\right) .
$$

Por outro lado,

$$
\{\theta[0]=-i\}=\left\{X_{-i+1}^{-1}=0^{i-1}, X_{-i}=1\right\}
$$

portanto

$$
\mathbb{Q}\left(X_{0}^{l-1}=a_{0}^{l-1}\right)=\sum_{i \geq 1} \mathbb{Q}\left(X_{-i}=1, X_{-i+1}^{-1}=0^{i-1}, X_{0}^{l-1}=a_{0}^{l-1}\right) .
$$


Agora, vamos utilizar a estrutura de $(\tau, p)$ para decompor $\mathbb{Q}\left(X_{-i}=1, X_{-i+1}^{-1}=0^{i-1}, X_{0}^{l-1}=a_{0}^{l-1}\right)$ em função das probabilidades de transição como sendo:

$$
\mathbb{Q}\left(X_{-i}=1\right) p(0 \mid 1) p(0 \mid 01) \ldots p\left(a_{0} \mid 0^{i-1} 1\right) p\left(a_{1} \mid a_{0} 0^{i-1} 1\right) \ldots p\left(a_{l-1} \mid a_{l-2} \ldots a_{0} 0^{i-1} 1\right),
$$

e finalmente, se denotamos $\mathbb{Q}\left(X_{i}^{i-|v|-1}=v\right.$ ) por $\mathbb{Q}(v)$ para todo $i$ e todo $v \in A^{|v|}$ (já que $\mathbb{Q}$ é estacionária):

$$
\mathbb{Q}\left(a_{0}^{l-1}\right)=\mathbb{Q}(1) \sum_{i \geq 1} \prod_{j=0}^{i-2}\left(1-p_{j}\right) \prod_{k=0}^{l-1} p\left(a_{k} \mid a_{0}^{k-1} 0^{i-1} 1\right)
$$

Para obter (4.4), precisamos explicitar $\mathbb{Q}(1)$. Basta utilizar o fato que $\mathbb{Q}(0)+\mathbb{Q}(1)=1$, e obtemos

$$
1=\mathbb{Q}(1)\left(1+\sum_{i \geq 1} \prod_{j=0}^{i-1}\left(1-p_{j}\right)\right)
$$

portanto

$$
\mathbb{Q}(1)=\frac{1}{1+\sum_{i \geq 1} \prod_{j=0}^{i-1}\left(1-p_{j}\right)} .
$$

Mais uma vez, o cálculo no caso $A$ qualquer é mais elaborado, porém não mais difícil.

Caso A enumerável. Como na prova anterior, podemos decompor segundo a última ocorrência do símbolo $b$

$$
\mathbb{Q}\left(X_{0}^{l-1}=a_{0}^{l-1}\right)=\sum_{i \geq 1} \sum_{a_{-i+1}^{-1} \in A_{\bar{b}}^{i-1}} \mathbb{Q}\left(X_{-i}=b, X_{-i+1}^{-1}=a_{-i+1}^{-1}, X_{0}^{l-1}=a_{0}^{l-1}\right)
$$

com $b \notin a_{-i+1}^{-1}$. Agora, utilizamos a estrutura de $(\tau, p)$ para decompor $\mathbb{Q}\left(X_{-i}=b, X_{-i+1}^{-1}=\right.$ $\left.a_{-i+1}^{-1}, X_{0}^{l-1}=a_{0}^{l-1}\right)$ em função das probabilidades de transição como sendo:

$$
\mathbb{Q}\left(X_{-i}=b\right) p\left(a_{-i+1} \mid b\right) p\left(a_{-i+2} \mid a_{-i+1} b\right) \ldots p\left(a_{0} \mid a_{-i+1}^{-1} b\right) p\left(a_{1} \mid a_{-i+1}^{0} b\right) \ldots p\left(a_{l-1} \mid a_{-i+1}^{l-2} b\right),
$$

e finalmente,

$$
\mathbb{Q}\left(a_{0}^{l-1}\right)=\mathbb{Q}(b) \sum_{i \geq 1} \sum_{a_{-i+1}^{-1} \in A_{\bar{b}}^{i-1}} \prod_{j=-i+1}^{l-1} p\left(a_{j} \mid a_{-i+1}^{j-1} b\right) .
$$


Para obter (4.3), precisamos explicitar $\mathbb{Q}(b)$. Basta utilizar o fato que $\sum_{a \in A} \mathbb{Q}(a)=1$, e obtemos

$$
1=\mathbb{Q}(b)\left(1+\sum_{a \in A_{\bar{b}}} \sum_{i \geq 1} \sum_{a_{-i+1}^{-1} \in A_{\bar{b}}^{i-1}} \prod_{j=-i+1}^{l-1} p\left(a_{j} \mid a_{-i+1}^{j-1} b\right)\right)
$$

portanto

$$
\mathbb{Q}(b)=\frac{1}{1+\sum_{a \in A_{\bar{b}}} \sum_{i \geq 1} \sum_{a_{-i+1}^{-1} \in A_{\bar{b}}^{i-1}} \prod_{j=-i+1}^{l-1} p\left(a_{j} \mid a_{-i+1}^{j-1} b\right)} .
$$

\subsection{Reversibilidade da cadeia esparsa}

Proposição 4.3. Seja $(\tau, p)$ a árvore esparsa com p verificando 4.1. Nesse caso, a lei $\mathbb{Q}$ da única cadeia estacionária compatível com $(\tau, p)$ é uma medida de probabilidade reversível para $(\tau, p)$.

Demonstração. Para provar isso consideramos um cilindro genérico. Podemos escrevê-lo

$$
0^{l_{1}} 10^{l_{2}} 1 \ldots 10^{l_{k-1}} 10^{l_{k}}
$$

Temos

$$
\mathbb{Q}\left(0^{l_{1}} 10^{l_{2}} 1 \ldots 10^{l_{k-1}} 10^{l_{k}}\right)=\mathbb{Q}\left(0^{l_{1}} 1\right) \prod_{m=2}^{k-1} \prod_{j=0}^{l_{m}-1}\left(1-p_{j}\right) p_{l_{m}} \prod_{j=0}^{l_{k}-1}\left(1-p_{j}\right)
$$

e do outro lado

$$
\mathbb{Q}\left(0^{l_{k}} 10^{l_{k-1}} 1 \ldots 10^{l_{2}} 10^{l_{1}}\right)=\mathbb{Q}\left(0^{l_{k}} 1\right) \prod_{m=2}^{k-1} \prod_{j=0}^{l_{m}-1}\left(1-p_{j}\right) p_{l_{m}} \prod_{j=0}^{l_{1}-1}\left(1-p_{j}\right)
$$

portanto basta mostrar que

$$
\mathbb{Q}\left(0^{l_{1}} 1\right) \prod_{j=0}^{l_{k}-1}\left(1-p_{j}\right)=\mathbb{Q}\left(0^{l_{k}} 1\right) \prod_{j=0}^{l_{1}-1}\left(1-p_{j}\right)
$$

Isso segue do fato que na medida estacionária sobre o alfabeto $\{0,1\}$ temos $\mathbb{Q}\left(0^{l} 1\right)=\mathbb{Q}\left(10^{l}\right)$ para 
todo $l \geq 0$ :

$$
\begin{aligned}
\mathbb{Q}\left(Y_{1}^{l+1}=10^{l}\right) & =\mathbb{Q}\left(Y_{1}={ }_{-}, Y_{2}^{l+1}=0^{l}\right)-\mathbb{Q}\left(Y_{1}^{l+1}=0^{l}\right) \\
& =\mathbb{Q}\left(Y_{1}^{l}=0^{l}, Y_{l+1}={ }_{-}\right)-\mathbb{Q}\left(Y_{1}^{l+1}=0^{l}\right)=\mathbb{Q}\left(0^{l} 1\right) .
\end{aligned}
$$

\subsection{Entropia da cadeia esparsa}

A razão da presente conta é a seguinte: apresentar o estudo mais completo possível da cadeia esparsa. Uma outra razão, que não discutimos nesta tese, está relacionada à noção de codificação finitária entre cadeias estocásticas. Na terminologia da teoria ergódica, a simulação perfeita apresentada na Seção 2.5 significa que a cadeia construída $\mathbf{X}(\mathbf{U})$ é uma codificação finitária da cadeia U. Esta área de pesquisa foi iniciada por Ornstein (1974). Mais tarde, Keane \& Smorodinsky (1977) provaram que existe uma codificação finitária de uma cadeia i.i.d. $(p, 1-p)$ para uma cadeia i.i.d. $(q, 1-q)$ desde que a entropia da primeira seja maior que a entropia do segundo. Um problema interessante seria generalizar este resultado para duas cadeias esparsas, com probabilidades de transição diferentes.

Denotamos por $H^{\tau}(p)$ a entropia da cadeia estacionária compatível com a árvore probabilística $(\tau, p)$. Também podemos fazer, no caso da árvore esparsa, a conta explícita da entropia da cadeia compatível:

Proposição 4.4. Se $(\tau, p)$ é a árvore esparsa com probabilidades de transição verificando (4.1) então

$$
H^{\tau}(p)=\mathbb{Q}(1) \sum_{i \geq 1} \prod_{j=0}^{i-2}\left(1-p_{j}\right) h\left(p_{i-1}\right)
$$

onde $h\left(p_{i-1}\right)=-p_{i-1} \log p_{i-1}-\left(1-p_{i-1}\right) \log \left(1-p_{i-1}\right)$ é a entropia de uma cadeia i.i.d. sobre $\{0,1\}$ e com distribuição $\left(p_{i-1}, 1-p_{i-1}\right)$.

Demonstração. Seja $\mathbf{X}$ a cadeia estacionária compatível com $(\tau, p), \mathbb{Q}$ a lei e $\mathbb{E}$ a esperança em relação a $\mathbb{Q}$, utilizamos a seguinte expressão da entropia da cadeia X (ver Cover \& Thomas (2006)):

$$
H^{\tau}(p)=\lim _{n \rightarrow \infty} \mathbb{E}\left(-\log \mathbb{Q}\left(X_{0} \mid X_{-1}, \ldots, X_{-n}\right)\right)
$$


Portanto

$$
\begin{gathered}
H^{\tau}(p)=-\lim _{n \rightarrow \infty} \sum_{a_{-n}^{-1} \in A^{n}} \sum_{a_{0} \in A} \mathbb{Q}\left(a_{-n}^{0}\right) \log \mathbb{Q}\left(X_{0}=a_{0} \mid X_{-n}^{-1}=a_{-n}^{-1}\right) \\
=-\lim _{n \rightarrow \infty}\left(\sum_{a_{-n}^{-1} \in A_{1}^{n}} \sum_{a_{0} \in A} \mathbb{Q}\left(a_{-n}^{0}\right) \log \mathbb{Q}\left(X_{0}=a_{0} \mid X_{-n}^{-1}=a_{-n}^{-1}\right)+\sum_{a_{0} \in A} \mathbb{Q}\left(0^{n} a_{0}\right) \log \mathbb{Q}\left(X_{0}=a_{0} \mid X_{-n}^{-1}=0^{n}\right)\right) .
\end{gathered}
$$

Chamamos a atenção sobre o fato que $A_{1}^{n}$ denota o conjunto das sequências de tamanho $n$ de símbolos em $A \backslash\{1\}$. O segundo termo escreve-se

$$
\mathbb{Q}\left(0^{n}\right) \sum_{a_{0} \in A} \mathbb{Q}\left(X_{0}=a_{0} \mid X_{-n}^{-1}=0^{n}\right) \log \mathbb{Q}\left(X_{0}=a_{0} \mid X_{-n}^{-1}=0^{n}\right) \leq \mathbb{Q}\left(0^{n}\right) \log 2
$$

que vai para 0 quando $n$ diverge, pois nas condições da proposição, sabemos que

$$
\lim _{n \rightarrow \infty} \mathbb{Q}\left(0^{n}\right)=0 .
$$

Agora, dividimos o conjunto das sequências $a_{-n}^{-1}$ de $A_{1}^{n}$ em $n$ subconjuntos, de acordo com a posição da última ocorrência do símbolo 1 em $a_{-n}^{-1}$, isto é,

$$
\begin{gathered}
\sum_{a_{-n}^{-1} \in A_{1}^{n}} \sum_{a_{0} \in A} \mathbb{Q}\left(a_{-n}^{0}\right) \log \mathbb{Q}\left(X_{0}=a_{0} \mid X_{-n}^{-1}=a_{-n}^{-1}\right) \\
=\sum_{i=1}^{n} \sum_{a_{-n}^{-i-1} \in A^{n-i}} \sum_{a_{0} \in A} \mathbb{Q}\left(a_{-n}^{-i-1} 10^{i-1} a_{0}\right) \log \mathbb{Q}\left(X_{0}=a_{0} \mid X_{-n}^{-1}=0^{i-1} 1 a_{-n}^{-i-1}\right) \\
=\sum_{i=1}^{n} \sum_{a_{-n}^{-i-1} \in A^{n-i}} \sum_{a_{0} \in A} \mathbb{Q}\left(a_{-n}^{-i-1} 10^{i-1} a_{0}\right) \log p\left(a_{0} \mid 0^{i-1} 1\right) .
\end{gathered}
$$

Utilizando a forma da árvore $\tau$ vemos que

$$
\mathbb{Q}\left(a_{-n}^{-i-1} 10^{i-1} a_{0}\right)=\mathbb{Q}\left(a_{-n}^{-i-1} 1\right) \prod_{j=0}^{i-2}\left(1-p_{j}\right) p\left(a_{0} \mid 0^{i-1} 1\right)
$$

$\log 0$

$$
\sum_{a_{-n}^{-1} \in A_{1}^{n}} \sum_{a_{0} \in A} \mathbb{Q}\left(a_{-n}^{0}\right) \log \mathbb{Q}\left(X_{0}=a_{0} \mid X_{-n}^{-1}=a_{-n}^{-1}\right)
$$




$$
=\sum_{i=1}^{n} \sum_{a_{-n}^{-i-1} \in A^{n-i}} \mathbb{Q}\left(a_{-n}^{-i-1} 1\right) \prod_{j=0}^{i-2}\left(1-p_{j}\right) \sum_{a_{0} \in A} p\left(a_{0} \mid 0^{i-1} 1\right) \log p\left(a_{0} \mid 0^{i-1} 1\right) .
$$

Como $\sum_{a_{-n}^{-i-1} \in A^{n-i}} \mathbb{Q}\left(a_{-n}^{-i-1} 1\right)=\mathbb{Q}(1)$, obtemos utilizando a notação $h\left(p_{i-1}\right)=-p_{i-1} \log p_{i-1}-$ $\left(1-p_{i-1}\right) \log \left(1-p_{i-1}\right)$ :

$$
\sum_{a_{-n}^{-1} \in A_{1}^{n}} \sum_{a_{0} \in A} \mathbb{Q}\left(a_{-n}^{0}\right) \log \mathbb{Q}\left(X_{0}=a_{0} \mid X_{-n}^{-1}=a_{-n}^{-1}\right)=-\mathbb{Q}(1) \sum_{i=1}^{n} \prod_{j=0}^{i-2}\left(1-p_{j}\right) h\left(p_{i-1}\right) .
$$

\subsection{Simulação perfeita}

Consideramos árvores de contextos probabilísticas tendo um contexto terminal (não necessariamente de tamanho 1). No caso em que os elementos de $\mathcal{W}$ são $\epsilon$-regulares sabemos fazer (o que não implica que não se pode fazer no caso contrário) um acoplamento desde o passado para construir uma amostra da cadeia distribuída de acordo com a medida estacionária. De acordo com o que dizemos no capítulo de preliminares (Seção 2.5), basta mostrar que a variável aleatória $\theta[m, n]$ é quase-certamente finita.

Teorema 4.5. Seja $(\tau, p)$ uma árvore probabilística tendo um contexto terminal $w$ que é também $\epsilon$-regular. Neste caso o algoritmo de simulação perfeita pára quase certamente depois de um número finito de etapas, isto é, temos para todo $-\infty<m \leq n<+\infty$

$$
\mathbb{P}(\theta[m, n]>-\infty)=1
$$

A prova deste teorema é particularmente simples e poderia ser omitida. No entanto ela nos permite introduzir o esquema da prova que é feita no capítulo 5 no caso das árvores mais gerais. Essa é a razão pela qual a incluímos.

Como $w$ é $\epsilon$-regular, temos $\mathcal{W} \subset \mathcal{E}$. Utilizando a partição descrita na Seção 2.5 (ver Figura 2.2) introduzimos uma nova cadeia $\overline{\mathbf{Z}}$ a valor em $\{1, \star\}$, definida da seguinte maneira:

$$
\bar{Z}_{m}=\left\{\begin{array}{ccc}
1 & \text { se } & U_{m|w|-i+1} \in J\left(w_{-i} \mid \emptyset\right), i=1, \ldots,|w| \\
\star & \text { senão }
\end{array}\right.
$$


Observaçoes 4.6. Assim definida, a cadeia $\overline{\mathbf{Z}}$ é i.i.d. com distribuição $\mathbb{P}\left(\bar{Z}_{m}=1\right)=1$ $\mathbb{P}\left(\bar{Z}_{m}=\star\right)=\epsilon^{|w|}$.

Definimos para todo $0 \leq n \leq+\infty$

$$
\bar{\theta}[0, n]=\max \left\{i \leq 0: \bar{Z}_{i}=1\right\}
$$

Temos o seguinte lema.

Lema 4.1. Para todo $0 \leq n \leq+\infty$

$$
(\bar{\theta}[0, n]-1)|w|+1 \leq \theta[0, n|w|] .
$$

Demonstração. Pela maneira que realizamos a simulação perfeita (ver Seção 2.5), e pela definição da cadeia $\overline{\mathbf{Z}}$ sabemos que

$$
X_{(\bar{\theta}[0, n]-1)|w|+1}^{\bar{\theta}[0, n]|w|}=w
$$

Portanto $\mathcal{L}\left(U_{(\bar{\theta}[0, n]-1)|w|+1}^{\bar{\theta}[0, n]|w|}\right)=1$. É claro que como $w$ é terminal, teremos $\mathcal{L}\left(U_{(\bar{\theta}[0, n]-1)|w|+1}^{i}\right)=1$ para todo $i \geq \bar{\theta}[0, n]|w|$, portanto $(\bar{\theta}[0, n]-1)|w|+1$ pertence ao conjunto

$$
\left\{i \leq-|w|+1: \mathcal{L}\left(U_{i}^{n|w|}\right)=1\right\} \subset\left\{i \leq m: \mathcal{L}\left(U_{i}^{n|w|}\right)=1\right\}
$$

e como $\theta[0, n|w|]$ é o máximo sobre este conjunto, o lema está provado.

Prova do Teorema 4.5. Para todo $-\infty<m \leq n<+\infty$, a invariância por translção de $\mathbf{U}$ implica que

$$
\theta[m, n] \stackrel{\mathcal{D}}{=} \theta[0, n-m]
$$

onde $\stackrel{\mathcal{D}}{=}$ denota a igualdade em distribuição das duas variáveis aleatórias, e pela Definição 2.9 de $\theta[m, n]$ temos

$$
\theta[0, n-m] \geq \theta\left[0,\left\lceil\frac{n-m}{|w|}\right\rceil|w|\right]
$$

Basta ver que pela observação 4.6 a cadeia $\overline{\mathbf{Z}}$ é ergódica, segue que $\bar{\theta}\left[0,\left|\frac{n-m}{|w|}\right|\right]$ é quase-certamente finito para todo $-\infty<m \leq n<+\infty$, e portanto esse é também o caso de $\theta\left[0,\left\lceil\frac{n-m}{|w|}\right\rceil|w|\right]$ e de 
$\theta[m, n]$.

Observamos aqui que a cadeia é regenerativa (já sabíamos disso) pois $\mathbb{P}(\theta[0,+\infty]=1)=$ $\mathbb{P}(\theta[1,+\infty]=1)$ (por invariância por translação de $\mathbf{U})$, e

$$
\mathbb{P}(\theta[0,+\infty]=0) \geq \epsilon^{|w|}>0
$$

Também a esperança do tamanho dos blocos é finita: ela é majorada por $|w| / \epsilon^{|w|}$. 


\section{Capítulo 5}

\section{Extensão para árvores gerais: resultados principais da tese}

Neste capítulo apresentamos o resultado principal da tese que é uma extensão do Teorema 4.5 para árvores sem contexto terminal. Agora, as árvores de contexto só têm que ter um "bom contexto". Na primeira seção apresentamos todas as definições e notações necessárias para definir o que é um bom contexto (Definição 5.1).

\subsection{Partição das árvores e bons contextos}

Seja $(\tau, p)$ uma árvore probabilística e $w$ um dos seus contextos. No capítulo anterior, definimos

$\mathcal{I}_{k}^{w}$ o conjunto das sequências introdutórias de tamanho $k$ para os contextos contendo $w$. Lembramos a definição:

$$
\mathcal{I}_{k}^{w}=\left\{\left(v_{-k}, \ldots, v_{-1}\right) \in A^{*}: v \in \tau \quad \text { and } \quad m^{w}(v)=k\right\}
$$

A cada sequência introdutória $s$ de $\mathcal{I}_{k}^{w}$ associamos $\mathcal{C}_{k}^{w}(s)$ o conjunto das sequências conclusivas dos contextos tendo $s$ como sequência introdutória, isto é,

$$
\mathcal{C}_{k}^{w}(s)=\left\{\left(v_{-|v|}, \ldots, v_{-k-|w|-1}\right) \in A^{*}: v \in \tau \quad \text { and } \quad v_{-k}^{-1}=s\right\}
$$

que é uma árvore de contextos com raíz em $s$. Denotamos a união sobre as sequências introdutórias $s$ de tamanho $k$ como

$$
\mathcal{C}_{k}^{w}:=\bigcup_{s \in \mathcal{I}_{k}^{w}} \mathcal{C}_{k}^{w}(s)=\left\{\left(v_{-|v|}, \ldots, v_{-k-|w|-1}\right) \in A^{*}: v \in \tau \quad \text { and } \quad m^{w}(v)=k\right\}
$$


Finalmente, $\ell^{w}(k)$ denota o tamanho máximo das sequências de $\mathcal{C}_{k}^{w}$

$$
\ell^{w}(k)=\sup \left\{|v|: v \in \mathcal{C}_{k}^{w}\right\}
$$

Quando $\mathcal{C}_{k}^{w}$ é vazio, $\ell^{w}(k)=0$.

Definição 5.1. Dada uma árvore de contextos $\tau$ não limitada, dizemos que um contexto $w \in \tau$, $|w|<+\infty$ é um bom contexto se para todo $k$

$$
\ell^{w}(k)<+\infty
$$

Na próxima seção, apresentamos alguns exemplos para se familiarizar com a noção de bom contexto.

\subsection{Exemplos de árvores com bons contextos}

Apresentamos exemplos de árvores sobre alfabetos de duas ou três letras para facilitar a apresentação das figuras. É claro que um contexto terminal é um bom contexto, neste caso temos $\ell^{w}(k)=0$ para todo $k$. Este é o caso das árvores representadas nas figuras 2.2, 4.2 e 4.1.

\subsubsection{Figura 2.3}

Como na árvore esparsa (ver Figura 2.1(b)), o conjunto $\mathcal{I}_{k}^{1}$ tem somente um elemento que é a sequência de $k$ zeros: $\mathcal{I}_{k}^{1}=0^{k}$. Os conjuntos $\mathcal{C}_{k}^{1}$ são por exemplo

$$
\begin{gathered}
\mathcal{C}_{1}^{1}=\mathcal{C}_{1}^{1}(0)=\{0,01,11\}, \\
\mathcal{C}_{3}^{1}=\mathcal{C}_{3}^{1}(000)=\{00,010,110,01,11\}, \\
\mathcal{C}_{5}^{1}=\mathcal{C}_{5}^{1}(00000)=\{\emptyset\} .
\end{gathered}
$$

Portanto, nesta notação um contexto $v$ tal que $m^{1}(v)=4$ (por exemplo 101110000) escreve-se

$$
\underbrace{1011}_{\in \mathcal{C}_{4}^{1}} 1 \underbrace{0000}_{\in \mathcal{I}_{4}^{1}}
$$


Observe também que neste caso simples, a função $\ell^{1}$ tem valores

$$
\ell^{1}(1)=2, \ell^{1}(2)=7, \ell^{1}(3)=3, \ell^{1}(4)=4, \ell^{1}(5)=0, \ldots
$$

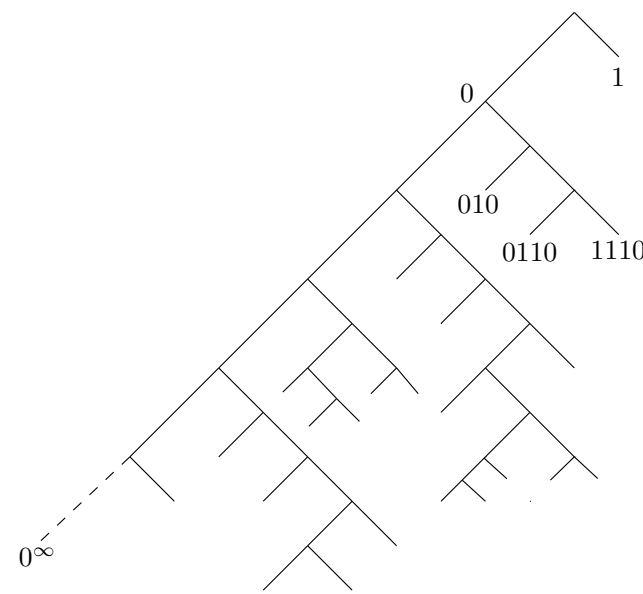

Figura 5.1: Árvore sobre $A=\{0,1\}$ tendo 1 como bom contexto

\subsubsection{Figura 5.2}

Nesta árvore, o menor bom contexto é 01. Para simplificar a figura, agrupamos os contextos tendo a mesma sequência introdutória sob a forma de pequenas árvores (os triângulos). Estas pequenas árvores são árvores de contexto também, de profundidade finita pois 01 é um bom contexto. Temos por exemplo

$$
\mathcal{C}_{4}^{01}=\mathcal{C}_{4}^{01}(0000) \cup \mathcal{C}_{4}^{01}(1100) \cup \mathcal{C}_{4}^{01}(1111)
$$

\subsubsection{Figura 5.3}

Neste caso o bom contexto é o 1 . Aqui também agrupamos os contextos tendo a mesma sequência introdutória sob a forma de pequenas árvores. Temos $\mathcal{I}_{k}^{1}=\{0,2\}^{k}$ e para todo $k$

$$
\mathcal{C}_{k}^{1}=\bigcup_{s \in\{0,2\}^{k}} \mathcal{C}_{k}^{1}(s)
$$




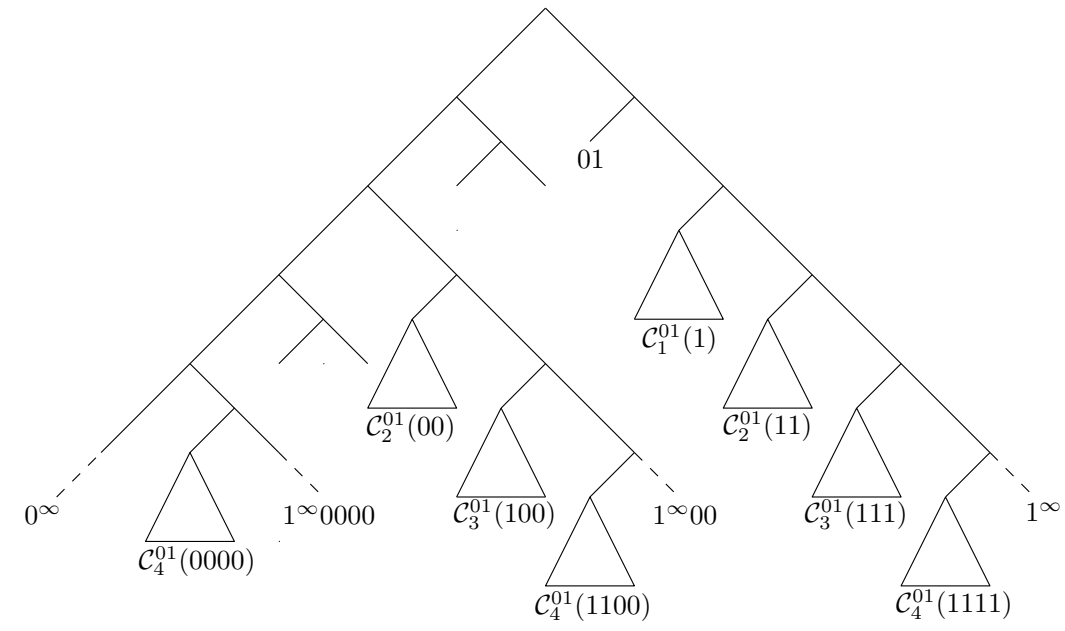

Figura 5.2: Árvore sobre $A=\{0,1\}$ tendo 01 como bom contexto

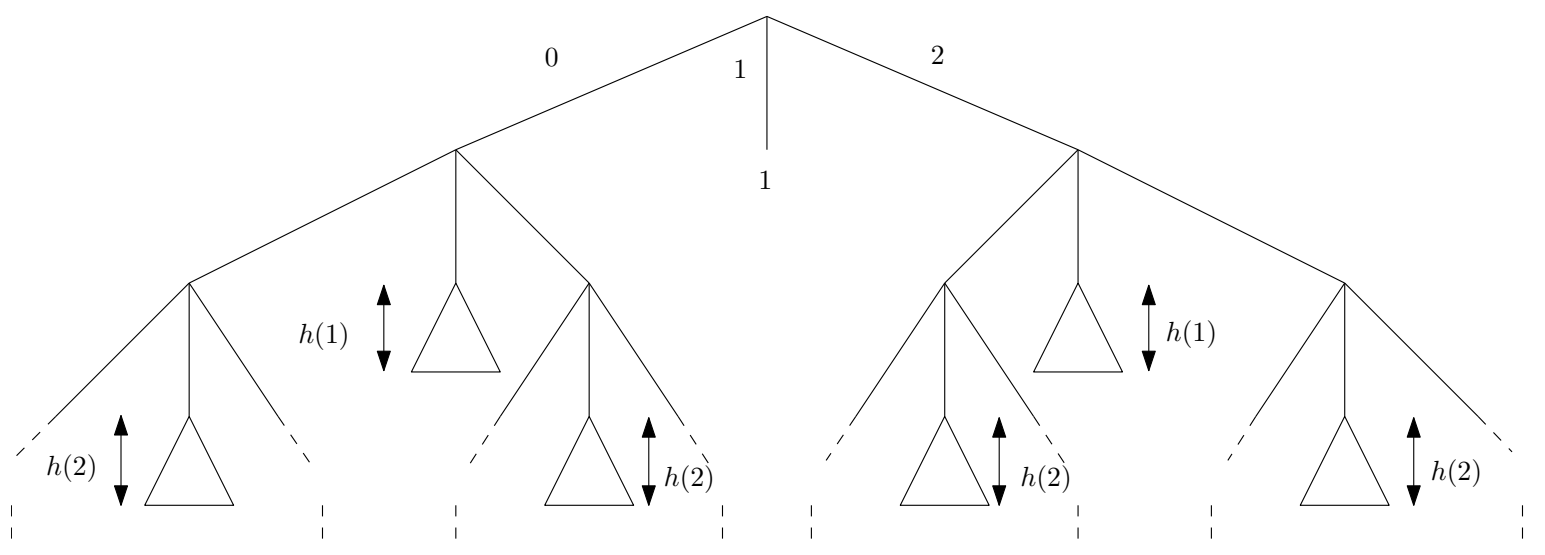

Figura 5.3: Árvore sobre $A=\{0,1,2\}$ tendo 1 como bom contexto

\subsection{Enunciado dos resultados}

Teorema 5.2. Seja uma árvore probabilística $(\tau, p)$ tendo um bom contexto $w$ que é também $\epsilon$ regular. Se

$$
\limsup _{k \rightarrow \infty} \frac{\log \left(\ell^{w}(k)\right)}{C_{\epsilon} k}<1
$$

com

$$
C_{\epsilon}:=\log \left[\left(\frac{1}{1-\epsilon^{|w|}}\right)^{1 /|w|}\right]>0,
$$

então o algoritmo de simulação perfeita é factível, isto é, temos para todo $-\infty<m \leq n<+\infty$

$$
\mathbb{P}(\theta[m, n]>-\infty)=1
$$


Esse teorema diz o seguinte. Se o bom contexto $w$ for $\epsilon$-regular, e se $\ell^{w}$ não cresce rápido demais (menos que uma exponencial em $k$ cuja taxa depende de $\epsilon$ ), então podemos fazer uma simulação perfeita da única cadeia estacionária compatível com $(\tau, p)$. Também podemos mostrar o seguinte.

Teorema 5.3. (Esquema regenerativo e perda de memória). Nas condições do Teorema 5.2 a cadeia $\mathbf{X}$ tem um esquema regenerativo, e o tamanho médio entre dois tempos de regeneração sucessivos é finito. Segue também que a perda de memória ocorre numa velocidade somável:

$$
\sum_{i \geq 0} \mathbb{P}(\theta[0]<-i)<+\infty
$$

Teorema 5.4 (Aproximação markoviana). Seja $(\tau, p)$ uma árvore probabilística verificando as condições do Teorema 5.2. Temos

$$
\bar{d}\left(\mathbf{X}, \mathbf{X}^{[k]}\right) \stackrel{k \rightarrow+\infty}{\longrightarrow} 0
$$

onde $\mathbf{X}$ denota a única cadeia estacionária compatível com $(\tau, p)$, e $\mathbf{X}^{[k]}$ sua aproximação Markoviana canônica.

Quando o bom contexto é uma letra ( $b$ por exemplo) do alfabeto $A$, definimos para todo $s$ em $\mathcal{I}_{i}^{b}$

$$
\begin{gathered}
p_{i}(b \mid s b)=\inf \left\{p(b \mid s b c): c \in \mathcal{C}_{i}^{b}(s)\right\}, \\
p_{i}(b)=\inf \left\{p_{i}(b \mid s b): s \in \mathcal{I}_{i}^{b}\right\} .
\end{gathered}
$$

Definimos também

$$
r^{b}(k)=\inf \left\{i \geq 1: \ell^{b}(i)>k\right\}
$$

que é a função inversa de $\ell^{b}$ (ambas a valor em $\mathbb{N}$ ).

Teorema 5.5. Seja $(\tau, p)$ uma árvore probabilística tendo um bom contexto $b \in A$ tal que

$$
\sum_{i \geq 1} \prod_{j=0}^{i-1}\left(1-p_{j}(b)\right)<+\infty .
$$

Se

$$
\sum_{i \geq 0} \prod_{j=0}^{r^{b}(i)-1}\left(1-p_{j}(b)\right)<+\infty
$$


então existe uma única cadeia estacionária compativel com $(\tau, p)$. Esta cadeia possui um esquema regenerativo.

Nesse último teorema, não assumimos a $\epsilon$-regularidade, por esta razão só sabemos mostrar a existência e a unicidade da cadeia compatível. Não sabemos fazer uma simulação perfeita destas cadeias. A hipótese deste teorema é a mais geral que conseguimos. No entanto ela não é muito intuitiva, porque ela envolve a função $r^{b}$. Apresentamos um corolário, cujo objetivo é tornar as hipóteses mais parecidas com aquelas do Teorema 5.2. O envelope inferior de uma função $f$ é a "maior" função monotonicamente crescente cujos valores são sempre inferiores ou iguais aos valores de $f$.

Corolario 5.6. Seja $(\tau, p)$ uma árvore probabilística tendo um bom contexto $b \in A$, tal que $p_{i}(b)>0$ para todo $i>0$. Denotamos por $\left\{p_{i}^{\prime}(b)\right\}_{i \geq 0}$ o envelope inferior de $\left\{p_{i}(b)\right\}_{i \geq 0}$. Se

$$
\sum_{i \geq 1} \prod_{j=0}^{i-1}\left(1-p_{j}^{\prime}(b)\right)<+\infty
$$

$e$

$$
\limsup _{k \rightarrow \infty} \frac{\log \left(\ell^{b}(k)\right)}{\log \left(\frac{1}{1-p_{k}^{\prime}(b)}\right)^{k}}<1,
$$

então existe uma única cadeia estacionária compatível com $(\tau, p)$. Esta cadeia possui um esquema regenerativo. 


\section{Capítulo 6}

\section{Prova do Teorema 5.2}

Começaremos este capítulo com algumas definições e um lema cujo objetivo é transformar o problema do estudo da variável $\theta$ num problema mais simples, o de estudar uma outra varíavel $\bar{\theta}$ que definimos a seguir. Na Seção 6.1 provamos este lema. Na Seção 6.2, estudamos a distribuição de $\bar{\theta}$. Somente na última parte do capítulo, provamos o Teorema 5.2.

Seja $(\tau, p)$ uma árvore de contextos probabilística que verifica as condições do Teorema 5.2, tendo $w$ como bom contexto. Se a função $\ell^{w}$ for limitada, então $(\tau, p)$ sería uma árvore com contexto terminal (que é um caso particular de bom contexto). Portanto, podemos sem perda de generalidade assumir que $\ell^{w}$ é não limitada. Por outro lado, a hipótese do teorema é que a função $\ell^{w}$ não cresca rápido demais. Isso nos permite assumir sem perda de generalidade que esta função é crescente (caso contrário, como ela é não limitada, podemos pegar o envelope superior de $\ell^{w}$, que é crescente). Resumindo, faremos a prova do teorema assumindo que $\ell^{w}$ é crescente e não limitada.

As hipóteses sobre a forma da árvore implicam que se $v$ é um contexto de $\tau$ tal que $w \in v$, temos

$$
|v| \leq m^{w}(v)+|w|+\ell^{w}\left(m^{w}(v)\right)
$$

Portanto, definindo o índice de tempo

$$
t(n)=\max \left\{k \leq n:(X(\mathbf{U}))_{k-|w|+1}^{k}=w\right\}
$$

e a função

$$
h^{w}(l):=l+|w|+\ell^{w}(l)
$$


temos para todo $(X(\mathbf{U}))_{-\infty}^{n}$ in $A^{-\mathbb{N}}$ (utilizando (6.1))

$$
\left|c_{\tau}\left((X(\mathbf{U}))_{-\infty}^{n}\right)\right| \leq h^{w}(n-t(n))
$$

Utilizamos a cadeia $\overline{\mathbf{Z}}$ (introduzida na Seção 4.5). Lembramos a sua definição

$$
\bar{Z}_{m}=\left\{\begin{array}{cc}
1 & \text { se } \\
\star & \text { caso contrário. }
\end{array}\right.
$$

Definimos também a variável aleatória

$$
\bar{t}(n):=\max \left\{k \leq n: \bar{Z}_{k}=1\right\}
$$

as novas funções

$$
\bar{\ell}(i):=\left\lceil\frac{\ell^{w}(i|w|)}{|w|}\right\rceil, \text { e } \bar{h}(i):=i+1+\bar{\ell}(i), i \geq 0
$$

e a variável aleatória

$$
\bar{\theta}[0, n]:=\max \left\{k \leq m: \bar{Z}_{k}=1 \text { e } \bar{h}(j-\bar{t}(j)) \leq j-k+1, j=k+1, \ldots, n\right\} .
$$

A Definição 6.6 pode ser comparada à Definição 4.7 da mesma variável no caso em que $w$ era um contexto terminal (Seção 4.5). A principal complicação vem do fato que não basta voltar até a última ocorrência do contexto $w$ (como era o caso na Definição 4.7).

Observaçoes 6.1. Chamamos a atenção sobre os seguintes fatos:

- Os índices de tempo $t(n)$ e $\bar{t}(n)$ são variáveis aleatórias, dependendo de $(X(\mathbf{U}))_{t(n)-|w|+1}^{n} e$ de $\overline{\bar{Z}_{\bar{t}}(n)}$.

- Temos sempre $t(i|w|) \geq \bar{t}(i)|w|$ que segue do fato que para todo $n$

$$
\bar{Z}_{n}=1 \Rightarrow(X(\mathbf{U}))_{(n-1)|w|+1}^{n|w|}=w
$$

Isso é uma consequência do algoritmo com o qual construímos a cadeia $\mathbf{X}(\mathbf{U})$. 
- No caso $|w|=1$ (supondo sem perda de generalidade que $w=1$ ), temos as seguinte equivalências: $\ell^{w} \equiv \bar{\ell}$ e $h^{w} \equiv \bar{h}$.

O seguinte lema justifica a utilização da nova variável $\bar{\theta}[0, n]$.

Lema 6.1. Para todo $n \geq 0$, sobre o conjunto $\{\mathbf{U}: \bar{\theta}[0, n]>-\infty\}$ temos que

$$
\theta[0, n|w|] \geq|w|(\bar{\theta}[0, n]-1)+1
$$

Com este lema, a situação se torna mais simples, porque $\bar{\theta}[0, n]$ é obtido a partir da cadeia $\overline{\mathbf{Z}}$ que é i.i.d., a valores em $\{1, \star\}$. Na seção que segue, provamos este lema, e na seção seguinte, mostramos que $\bar{\theta}[0, n]$ é quase-certamente finito para todo $0<n \leq+\infty$.

\subsection{Prova do Lema 6.1}

A prova deste lema é trabalhosa.

Prova do Lema 6.1. Primeiro, queremos mostrar que

$$
|w|(\bar{\theta}[0, n]-1)+1 \in\left\{k \leq 0: \mathcal{L}\left(U_{k}^{n|w|}\right)=1\right\} .
$$

O algoritmo com o qual construímos $\mathbf{X}(\mathbf{U})$ é tal que se

$$
U_{m|w|-i+1} \in J\left(w_{-i} \mid \emptyset\right), \quad i=1, \ldots,|w|
$$

então $(X(\mathbf{U}))_{(m-1)|w|+1}^{m|w|}=w$ e $\mathcal{L}\left(U_{(m-1)|w|+1}^{|w| m}\right)=1$. Pela definição de $\bar{\theta}[0, n]$ temos $\bar{Z}_{\bar{\theta}[0, n]}=1$. Segue que $(X(\mathbf{U}))_{(\bar{\theta}[0, n]-1)|w|+1}^{|w| \bar{\theta}[0, n]}=w$ e $\mathcal{L}\left(U_{(\bar{\theta}[0, n]-1)|w|+1}^{|w| \bar{\theta}[0, n]}\right)=1$.

Supomos que para algum $i, \bar{\theta}[0, n]+1 \leq i \leq n$ temos $\mathcal{L}\left(U_{(\bar{\theta}[0, n]-1)|w|+1}^{|w|(i-1)}\right)=1$. Vamos mostrar que $\mathcal{L}\left(U_{(\bar{\theta}[0, n]-1)|w|+1}^{|w| i}\right)=1$. Por indução, isso provará que $\mathcal{L}\left(U_{(\bar{\theta}[0, n]-1)|w|+1}^{n|w|}\right)=1$, e portanto que vale (6.7).

1. Se $\bar{h}(i-\bar{t}(i))=1$. Isso significa que $\bar{Z}_{i}=1$, segue que $\mathcal{L}\left(U_{(i-1)|w|+1}^{|w| i}\right)=1$, e portanto que $\mathcal{L}\left(U_{(\bar{\theta}[0, n]-1)|w|+1}^{|w| i}\right)=1$.

2. Se $\bar{h}(i-\bar{t}(i))>1$. Isso significa que $\bar{Z}_{i}=\star$. Portanto, vamos primeiro mostrar que para todo 
$j,(i-1)|w| \leq j \leq i|w|-1$, se

$$
\mathcal{L}\left(U_{(\bar{\theta}[0, n]-1)|w|+1}^{j}\right)=1
$$

então

$$
\left|c_{\tau}\left((X(\mathbf{U}))_{(\bar{\theta}[0, n]-1)|w|+1}^{j}\right)\right| \leq j-(\bar{\theta}[0, n]-1)|w| .
$$

Analizamos em duas partes:

- No tempo $j=(i-1)|w|$. Já sabemos que $\mathcal{L}\left(U_{(\bar{\theta}[0, n]-1)|w|+1}^{j}\right)=1$.

- Se $i-1=\bar{\theta}[0, n]$ então $(X(\mathbf{U}))_{(\bar{\theta}[0, n]-1)|w|+1}^{j}=(X(\mathbf{U}))_{(\bar{\theta}[0, n]-1)|w|+1}^{\bar{\theta}[0, n]|w|}=w$, e portanto

$$
\left|c_{\tau}\left((X(\mathbf{U}))_{(\bar{\theta}[0, n]-1)|w|+1}^{j}\right)\right|=|w|=j-(\bar{\theta}[0, n]-1)|w| .
$$

- Se $i-1>\bar{\theta}[0, n]$ observamos que

$$
t((i-1)|w|) \geq \bar{t}(i-1)|w|
$$

portanto temos $(i-1)|w|-t((i-1)|w|) \leq((i-1)-\bar{t}(i-1))|w| \mathrm{e}$

$$
\begin{aligned}
& h^{w}((i-1)|w|-t((i-1)|w|)):=(i-1)|w|-t((i-1)|w|)+|w|+\ell^{w}((i-1)|w|-t((i-1)|w|)) \\
& \leq((i-1)-\bar{t}(i-1))|w|+|w|+\ell^{w}(((i-1)-\bar{t}(i-1))|w|) \leq|w| \bar{h}((i-1)-\bar{t}(i-1)) .
\end{aligned}
$$

Esta última desigualdade segue das definições de $\bar{\ell}$ e $\bar{h}$ (ver (6.5)). No presente caso, temos $\bar{\theta}[0, n]+1 \leq i-1 \leq n-1$, e sabemos pela Definição 6.6 de $\bar{\theta}[0, n]$ que

$$
\bar{h}((i-1)-\bar{t}(i-1)) \leq(i-1)-\bar{\theta}[0, n]+1 .
$$

\section{Portanto,}

$$
\left|c_{\tau}\left((X(\mathbf{U}))_{(\bar{\theta}[0, n]-1)|w|+1}^{j}\right)\right| \leq|w|((i-1)-\bar{\theta}[0, n]+1)=j-|w|(\bar{\theta}[0, n]-1) .
$$

- Nos tempos $j$ tais que $(i-1)|w|+1 \leq j \leq i|w|-1$. Supomos que $\mathcal{L}\left(U_{(\bar{\theta}[0, n]-1)|w|+1}^{j}\right)=1$, 
temos (lembrando que $\bar{Z}_{i}=\star$ )

$$
j-t(j) \leq j-|w| \bar{t}(i)
$$

e em particular

$$
j-t(j)<i|w|-|w| \bar{t}(i)
$$

Utilizamos estas duas majorações agora para majorar $h^{w}(j-t(j))$ :

$$
\begin{aligned}
& h^{w}(j-t(j)):=j-t(j)+|w|+\ell^{w}(j-t(j)) \\
& \quad \leq j-|w| \bar{t}(i)+|w|+\ell^{w}(|w|(i-\bar{t}(i))) .
\end{aligned}
$$

Pela definição de $\bar{\ell}$, obtemos

$$
h^{w}(j-t(j)) \leq j-|w| \bar{t}(i)+|w|+|w| \bar{\ell}(i-\bar{t}(i)):=j-i|w|+|w| \bar{h}(i-\bar{t}(i))
$$

Sabemos pela definição de $\bar{\theta}[0, n]$ que quando $\bar{\theta}[0, n]+1 \leq i \leq n$

$$
\bar{h}(i-\bar{t}(i)) \leq i-\bar{\theta}[0, n]+1
$$

$\log 0$

$$
h^{w}(j-t(j)) \leq j-i|w|+|w|(i-\theta[\overline{0}, n]+1)=j-|w|(\bar{\theta}[0, n]-1) .
$$

Acabamos de mostrar que para todo $j,(i-1)|w| \leq j \leq i|w|-1$, se

$$
\mathcal{L}\left(U_{(\bar{\theta}[0, n]-1)|w|+1}^{j}\right)=1
$$

então

$$
\left|c_{\tau}\left((X(\mathbf{U}))_{(\bar{\theta}[0, n]-1)|w|+1}^{j}\right)\right| \leq j-(\bar{\theta}[0, n]-1)|w| .
$$

Como no tempos $(i-1)|w|$ sabemos por hipótese que $\mathcal{L}\left(U_{(\bar{\theta}[0, n]-1)|w|+1}^{(i-1)|w|}\right)=1$, então por 
indução, temos

$$
\mathcal{L}\left(U_{(\bar{\theta}[0, n]-1)|w|+1}^{|w| i}\right)=1
$$

Portanto, já mostramos que

$$
|w|(\bar{\theta}[0, n]-1)+1 \in\left\{k \leq 0: \mathcal{L}\left(U_{k}^{n|w|}\right)=1\right\} .
$$

Como $\theta[0, n|w|]$ é o máximo sobre este conjunto, então

$$
\theta[0, n|w|] \geq|w|(\bar{\theta}[0, n]-1)+1 .
$$

Figura 6.1 Esta figura ilustra o Lema 6.1 utilizando para $h^{w}$ os valores:

$$
\begin{gathered}
h^{w}(1)=4, h^{w}(2)=7, h^{w}(3)=8, h^{w}(4)=10, \\
h^{w}(5)=12, h^{w}(6)=16 .
\end{gathered}
$$

A função $\bar{h}$ tem os seguintes valores, calculados $\operatorname{com} \bar{h}(i):=i+1+\left\lceil\frac{\ell^{w}(i|w|)}{|w|}\right\rceil$ :

$$
\begin{aligned}
& \bar{h}(1)=2+\left\lceil\frac{\ell^{w}(3)}{3}\right\rceil=2+\left\lceil\frac{2}{3}\right\rceil=3, \\
& \bar{h}(2)=3+\left\lceil\frac{\ell^{w}(6)}{3}\right\rceil=3+\left\lceil\frac{7}{3}\right\rceil=6,
\end{aligned}
$$

e, supondo que $\ell^{w}(9)=16$ (isto é que $h^{w}(28)$ )

$$
\bar{h}(3)=4+\left\lceil\frac{\ell^{w}(9)}{3}\right\rceil=3+\left\lceil\frac{16}{3}\right\rceil=9 .
$$

A sequência de cima representa as ocorrências de w's auto-definidos obtidos a partir da sequência U, e que portanto, aparecem na sequência $\mathbf{X}(\mathbf{U})$. As setas de cima representam o tamanho $h^{w}(i-t(i))$. No caso dos tempos autodefinidos, desenhamos pequenos laços. Como não damos os detalhes dos valores de $\mathbf{U}$, não podemos achar o tempo $\theta[0, n|w|]$ nesta figura. No entanto, podemos ver que 
a construção é possível a partir do tempo que denotamos por $k$, porque nenhuma seta ultrapasse este tempo. Em outras palavras, quando $(X(\mathbf{U}))_{i}, k \leq i \leq n|w|$ não é autodefinido, sabemos que o contexto $c_{\tau}\left((X(\mathbf{U}))_{k}^{i-1}\right)$ é definido, e portanto que podemos construir $(X(\mathbf{U}))_{i}$ utilizando $c_{\tau}\left((X(\mathbf{U}))_{k}^{i-1}\right)$. Como isso é o caso para $i, k \leq i \leq n|w|$, então

$$
\mathcal{L}\left(U_{k}^{n|w|}\right)=1
$$

A sequência de baixo representa $\overline{\mathbf{Z}}$, e as setas que saem dos tempos $i$ com estrelas representam os tamanhos $\bar{h}(i-\bar{t}(i))$. Podemos encontrar a partir deste desenho o tempo $\bar{\theta}[0, n]$. Em particular, podemos ver que a construção da cadeia $\mathbf{X}(\mathbf{U})$ é possível a partir do tempo $|w|(\theta[0, n]-1)+1$, porque na parte de cima, de novo, nenhuma seta vai ultrapassar este tempo:

$$
\mathcal{L}\left(U_{|w|(\theta[0, n]-1)+1}^{n|w|}\right)=1
$$

\subsection{Estudo da variável $\bar{\theta}[0, n]$}

Vamos mudar um pouco a definição desta variável:

$$
\bar{\theta}[0, n]:=\max \left\{k \leq m-\sigma+1: \bar{Z}_{k}^{k+\sigma-1}=1^{\sigma} \text { e } \bar{h}(j-\bar{t}(j)) \leq j-k+1, j=k+\sigma, \ldots, n\right\}
$$

O Lema 6.1 segue válido, simplesmente obrigamos a ocorrência de $\sigma$ 1's consecutivos. Dessa maneira, este novo $\bar{\theta}$ é sempre menor ou igual ao antigo. Para poder estudar a distribuição de $\bar{\theta}[0, n]$, precisamos de mais algumas definições. Primeiro, definimos a função $\bar{r}$ para $k \geq 0$ como

$$
\bar{r}(k)=\inf \{i \geq 1: \bar{\ell}(i)>k\}
$$

É a função inversa da função $\bar{\ell}$ (ambas a valor em $\mathbb{N}$ ).

Segundo, definimos para todo $n$ em $\mathbb{Z}$, uma nova cadeia $\mathbf{C}^{(n)}$ que é construída utilizando $\left(\bar{Z}_{i}\right)_{i \geq n+1}$ 


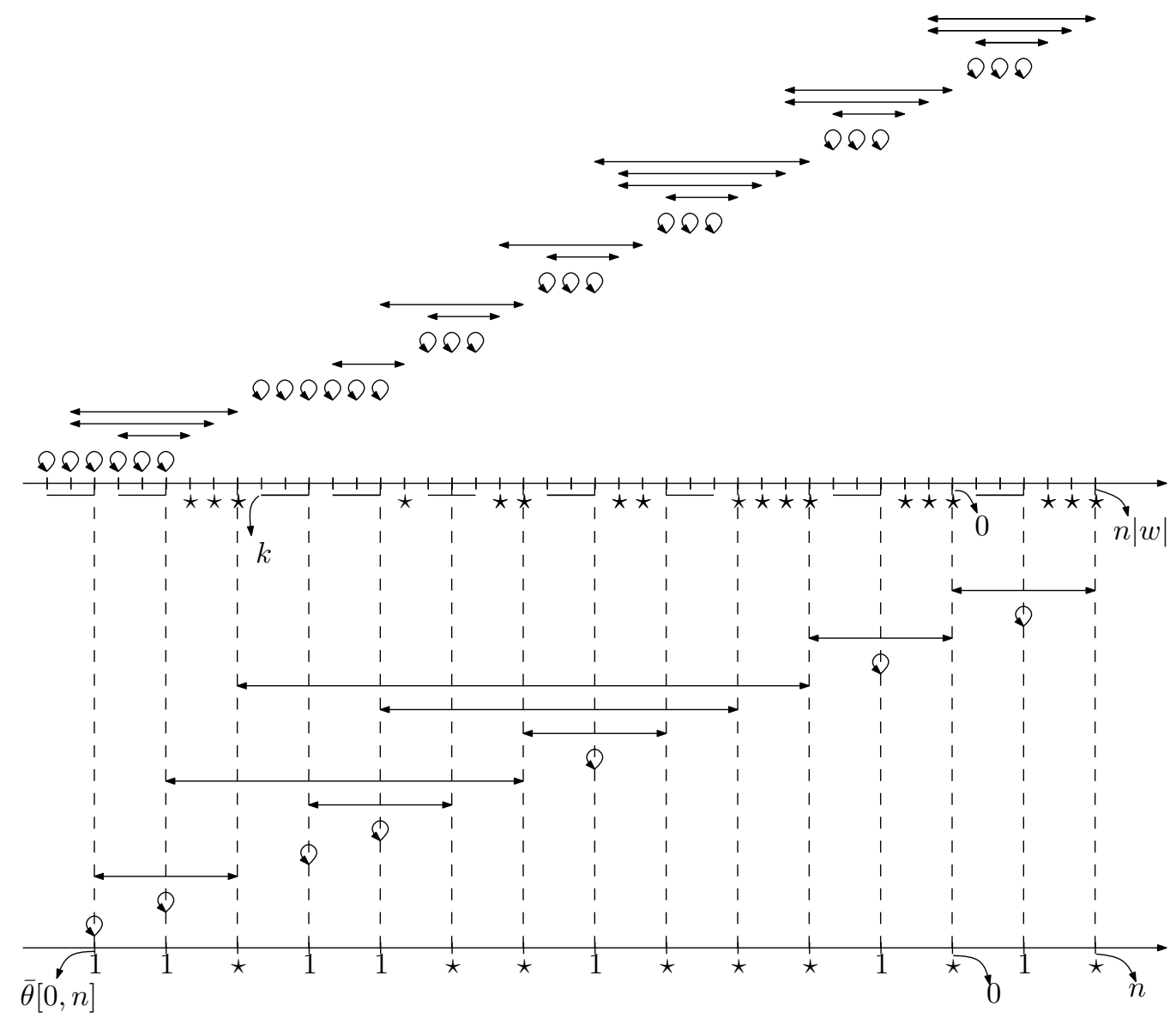

Figura 6.1: Ilustração da variável $\bar{\theta}[0, n]$ construída utilizando $\overline{\mathbf{Z}}$.

da seguinte maneira. Tomamos $C_{i}^{(n)}=0$ para todo $i \leq n \mathrm{e}$

$$
C_{i}^{(n)}=\mathbf{1}\left\{\bar{Z}_{i}=1\right\} \quad \text { para } i=n+1, \ldots, n+\sigma-1,
$$

no tempo $n+\sigma$, tomamos (veremos no Lema 6.3 a razão)

$$
C_{n+\sigma}^{(n)}=\mathbf{1}\left\{\bar{Z}_{n+\sigma}=1\right\} \bar{r}(\sigma-1)
$$

e para $i \geq n+\sigma+1$

$$
C_{i}^{(n)}=\min \{j \geq 0: \bar{h}(i+j-\bar{t}(i))>i-n+j\} .
$$

Lembramos que a aleatoriedade está em $\bar{t}(i)$ porque este depende da cadeia $\left(\bar{Z}_{n}\right)_{n \leq i}$. O próximo 
lema explica como esta nova cadeia e $\bar{\theta}[0, n]$ se relacionam.

Lema 6.2. Para todo $l \geq \sigma-1$ e todo $0 \leq n \leq+\infty$

$$
\{\bar{\theta}[0, n]<-l\}=\bigcap_{i=-l-1}^{-\sigma} \bigcup_{k=i+1}^{n}\left\{C_{k}^{(i)}=0\right\}
$$

Demonstração. Segue das definições. Observamos que pela definição da cadeia $\mathbf{C}^{(i)}$ temos

$$
\bigcap_{k=i+1}^{n}\left\{C_{k}^{(i)} \geq 1\right\}=\left\{\bar{Z}_{i+1}^{i+\sigma}=1^{\sigma}\right\} \cap \bigcap_{k=i+\sigma+1}^{n}\{\bar{h}(k-\bar{t}(k)) \leq k-i\}
$$

portanto

$$
\bigcup_{k=i+1}^{n}\left\{C_{k}^{(i)}=0\right\}=\left\{\bar{Z}_{i+1}^{i+\sigma} \neq 1^{\sigma}\right\} \cup \bigcup_{k=i+\sigma+1}^{n}\{\bar{h}(k-\bar{t}(k))>k-i\}
$$

$\mathrm{e}$

$$
\bigcap_{i=-l-1}^{-\sigma} \bigcup_{k=i+1}^{n}\left\{C_{k}^{(i)}=0\right\}=\bigcap_{i=-l-1}^{-\sigma}\left\{\left\{\bar{Z}_{i+1}^{i+\sigma} \neq 1^{\sigma}\right\} \cup \bigcup_{k=i+\sigma+1}^{n}\{\bar{h}(k-\bar{t}(k))>k-i\}\right\}
$$

De um outro lado, temos por (6.8)

$$
\{\bar{\theta}[0, n]<-l\}=\bigcap_{j=-l+\sigma-1}^{0}\left\{\left\{\bar{Z}_{j-\sigma+1}^{j} \neq 1^{\sigma}\right\} \cup \bigcup_{k=j+1}^{n}\{\bar{h}(k-\bar{t}(k))>k-j+\sigma\},\right.
$$

e tomanto $i=j-\sigma$ obtemos

$$
\{\bar{\theta}[0, n]<-l\}=\bigcap_{i=-l-1}^{-\sigma}\left\{\left\{\bar{Z}_{i+1}^{i+\sigma} \neq 1^{\sigma}\right\} \cup \bigcup_{k=i+\sigma+1}^{n}\{\bar{h}(k-\bar{t}(k))>k-i\}\right\} .
$$

O próximo lema é o lema chave da prova do teorema. Ele permite uma definição alternativa e mais apropriada da cadeia $\mathbf{C}^{(n)}$.

Lema 6.3. Para todo $n$, temos para $i \geq n+\sigma$

$$
C_{i}^{(n)}=\left\{\begin{array}{ccccc}
0 & \text { se } & \bar{Z}_{i} \neq 1 & \text { e } & C_{i-1}^{(n)}=0 \\
c-1 & \text { se } & \bar{Z}_{i} \neq 1 & \text { e } & C_{i-1}^{(n)}=c \geq 1 \\
\bar{r}(i-n-1) & \text { se } & \bar{Z}_{i}=1 . & &
\end{array}\right.
$$


Demonstração. No tempo $n+\sigma$, isso é claro porque

$$
C_{n+\sigma}^{(n)}=\mathbf{1}\left\{\bar{Z}_{n+\sigma}=1\right\} \bar{r}(\sigma-1) \quad \text { e } \quad C_{n+\sigma-1}^{(n)}=\mathbf{1}\left\{\bar{Z}_{n+\sigma-1}=1\right\}
$$

Para $i \geq n+\sigma+1, C_{i}^{(n)}$ é o menor inteiro positivo $j$ tal que

$$
\bar{h}((i+j)-\bar{t}(i))>i+j-n .
$$

Vamos proceder caso por caso.

Caso 1. Se $\bar{Z}_{i} \neq 1$ então $\bar{t}(i)=\bar{t}(i-1)$, separamos em dois subcasos.

- $C_{i-1}^{(n)}=0$. Isso significa que (6.12) vale no tempo $i-1$ com $j=0$ :

$$
A:=\bar{h}((i-1)-\bar{t}(i-1))>(i-1)-n .
$$

Calculamos:

$$
\bar{h}(i-\bar{t}(i))=\bar{h}(i-\bar{t}(i-1))=1+(i-1)-\bar{t}(i-1)+1+\bar{\ell}(i-\bar{t}(i-1)),
$$

e adicionando e substraindo $\bar{\ell}((i-1)-\bar{t}(i-1))$ obtemos

$$
\bar{h}(i-\bar{t}(i))=A+\bar{\ell}(i-\bar{t}(i-1))-\bar{\ell}((i-1)-\bar{t}(i-1))+1 .
$$

Então por (6.13)

$$
\bar{h}(i-\bar{t}(i))>i-n+\bar{\ell}(i-\bar{t}(i-1))-\bar{\ell}((i-1)-\bar{t}(i-1)),
$$

e como $\bar{\ell}$ é não decrescente, obtemos finalmente

$$
\bar{h}(i-\bar{t}(i))>i-n,
$$

o que significa que $C_{i}^{(n)}=0$. 
- $C_{i-1}^{(n)}=c \geq 1$. Isso significa que $c$ é o menor número inteiro verificando (6.12) no tempo $i-1$ :

$$
B:=\bar{h}((i-1+c)-\bar{t}(i-1))>(i-1+c)-n .
$$

Supondo que $j=c-1$, obtemos

$$
\bar{h}((i+j)-\bar{t}(i))=\bar{h}((i+c-1)-\bar{t}(i))=\bar{h}((i+c-1)-\bar{t}(i-1)),
$$

em que o último termo é igual a $B$, portanto por (6.14)

$$
\bar{h}((i+c-1)-\bar{t}(i))>i+(c-1)-n
$$

o que significa que $j=c-1$ verifica (6.12). No entanto, temos que mostrar que esse é o menor valor de $j$ verificando (6.12). Para isso, vamos supor que para algum $d<c-1$, temos

$$
\bar{h}((i+d)-\bar{t}(i))>i+d-n .
$$

Então como $\bar{t}(i)=\bar{t}(i-1)$ podemos re-escrever a desigualdade anterior como

$$
\bar{h}((i-1+d+1)-\bar{t}(i-1))>i-1+d+1-n,
$$

o que significa que no tempo $i-1$, o valor $j=d+1$ verifica (6.12). E como $d+1<c$, isso significa que $c$ não é o menor valor de $j$ verificando (6.12), e obtemos uma contradição com o caso presente (ver (6.14)). Portanto $c-1$ é o menor valor de $j$ verificando (6.12), e

$$
C_{i}^{(n)}=c-1
$$

Caso 2. Quando $\bar{Z}_{i}=1$ então $\bar{t}(i)=i$, o menor $j$ verificando (6.12) satisfaz

$$
\bar{\ell}(j)>i-n-1 \text {. }
$$


Pela definição de $\bar{r}$ (ver (6.9)), este número é $\bar{r}(i-n-1)$, portanto

$$
C_{i}^{(n)}=\bar{r}(i-n-1)
$$

Esta cadeia $\mathbf{C}^{(n)}$ é uma cadeia de Markov não homogênea. Preferimos (só assim conseguimos obter resultados mais gerais) estudar uma outra cadeia $\mathbf{D}^{(n)}$ definida da seguinte maneira: $D_{i}^{(n)}=0$ para $i \leq n$ e para $i>n$

$$
D_{i}^{(n)}=\left\{\begin{array}{ccccc}
0 & \text { se } & \bar{Z}_{i} \neq 1 & \text { e } & i \leq \sigma+i^{\prime} \\
c-1 & \text { se } & \bar{Z}_{i} \neq 1 & \text { e } & i>\sigma+i^{\prime} \\
1 & \text { se } & \bar{Z}_{i}=1 & \text { e } & i<\sigma+i^{\prime} \\
\bar{r}\left(i-i^{\prime}-1\right) & \text { se } & \bar{Z}_{i}=1 & \text { e } & i \geq \sigma+i^{\prime}
\end{array}\right.
$$

onde $1 \leq c=D_{i-1}^{(n)}$ e $i^{\prime}:=\max \left\{l<i: D_{l}^{(n)}=0\right\}$. Tomamos $D_{i-1}^{(n)}=c \geq 1$ quando $i>\sigma+i^{\prime}$ pois nesse caso $D_{i-1}^{(n)}$ tem valor 1 ou $\bar{r}\left(i-i^{\prime}-2\right) \geq \bar{r}(\sigma-1) \geq 1$. Ilustramos as duas cadeias $\mathbf{C}^{(n)}$ e $\mathbf{D}^{(n)}$ na Figura 6.2. Observamos que esta cadeia é não markoviana (as transições no tempo $i$ dependem do passado até o tempo $i^{\prime}$ ). No entanto, ela é regenerativa porque ela não depende do que aconteceu nos tempos anteriores ao tempo $i^{\prime}$. Tiramos proveito desse fato para estudar esta cadeia no Lema 6.6. Antes disso, precisamos de dois lemas.

Figura 6.2 Ilustramos as cadeias $\mathbf{C}^{(n)}, \mathbf{C}^{\left(n^{\prime}\right)}, \mathbf{D}^{(n)}$ e $\mathbf{D}^{\left(n^{\prime}\right)}$, todas construídas com a cadeia $\overline{\mathbf{Z}}$. Neste caso particular, $\sigma=3$ e a função $\bar{r}$ tem valores indicados em (6.16). Podemos ver as diferenças entre as duas cadeias: por exemplo a cadeia $\mathbf{D}^{(i)}, i=n, n^{\prime}$ começa a subir para o estado $\bar{r}(\sigma-1)=\bar{r}(2)$ somente após a ocorrência de $\sigma$ 1's consecutivos. Esta figura ilustra dois fatos: o fato que $C_{i}^{(n)} \geq D_{i}^{(n)}$ para todo $i$ e o fato (6.18) que diz que $\mathbf{D}^{(n)}$ e $\mathbf{D}^{\left(n^{\prime}\right)}$ coalescem após o primeiro 


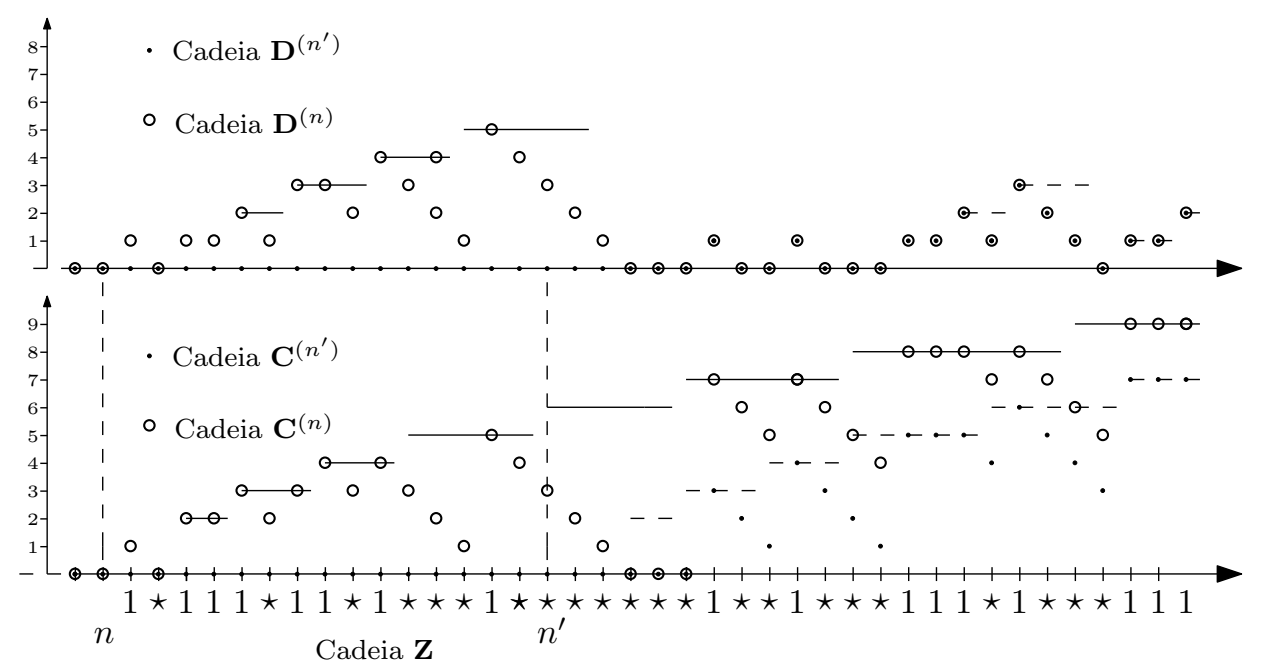

Figura 6.2: As cadeias $\mathbf{C}^{(n)}, \mathbf{C}^{\left(n^{\prime}\right)}, \mathbf{D}^{(n)}$ e $\mathbf{D}^{\left(n^{\prime}\right)}$ construídas utilizando $\overline{\mathbf{Z}}$ e os valores (6.16) para $\bar{r}$.

tempo em que $\mathbf{D}^{(n)}$ toca 0 . Na Figura 6.2 , a função $\bar{r}$ tem os seguintes valores.

$$
\begin{gathered}
\bar{r}(2)=\bar{r}(3)=2 \\
\bar{r}(4)=\bar{r}(5)=\bar{r}(6)=3 \\
\bar{r}(7)=\bar{r}(8)=\bar{r}(9)=4 \\
\bar{r}(10)=\bar{r}(11)=\bar{r}(12)=\bar{r}(13)=\bar{r}(14)=5 \\
\bar{r}(15)=\bar{r}(16)=\bar{r}(17)=\bar{r}(18)=\bar{r}(19)=6 \\
\bar{r}(20)=\bar{r}(21)=\bar{r}(22)=\bar{r}(23)=\bar{r}(24)=\bar{r}(25)=7
\end{gathered}
$$

Lema 6.4. Para todo n, temos

$$
C_{i}^{(n)} \geq D_{i}^{(n)}, \quad \forall i \in \mathbb{Z}
$$

Demonstração. Veja a Figura 6.2. Primeiro observamos que para todo $i \geq n+1$, temos $i^{\prime} \geq n$. Portanto, pela definição das cadeias, sabemos que elas são iguais quando $i<n+\sigma$. Para $i \geq n+\sigma$, basta observar que sempre que a cadeia $\mathbf{C}^{(n)}$ decresce de 1 (ou fica em 0 ), a cadeia $\mathbf{D}^{(n)}$ decresce de 1 (ou fica em 0); e que sempre que a cadeia $\mathbf{D}^{(n)}$ cresce, a cadeia $\mathbf{C}^{(n)}$ cresce também. Além disso, quando $\mathbf{C}^{(n)}$ e $\mathbf{D}^{(n)}$ crescem, $\mathbf{C}^{(n)}$ sempre vai para um estado superior porque como

$$
i-i^{\prime}-1 \leq i-n-1
$$


e como $\bar{r}$ é uma função crescente, temos sempre

$$
\bar{r}\left(i-i^{\prime}-1\right) \leq \bar{r}(i-n-1) .
$$

Denotamos para todo $k \geq 1, u_{k}:=\mathbb{P}\left(D_{k}^{(0)}=0\right)$.

Lema 6.5. Para todo $l \geq \sigma-1$ e $n \geq 0$

$$
\mathbb{P}(\bar{\theta}[0, n]<-l) \leq \sum_{k=l-\sigma+2}^{l+n+1} u_{k}
$$

Demonstração. Pela definição da cadeia $\mathbf{D}^{(n)}$, para quaisquer dois inteiros $-\infty<n_{1} \leq n_{2}<+\infty$

$$
D_{i}^{\left(n_{1}\right)} \geq D_{i}^{\left(n_{2}\right)}, \forall i \in \mathbb{Z}
$$

Além disso, se $D_{i}^{(n)}=0$ para algum $i>n$, temos $D_{j}^{(n)}=D_{j}^{(i)}$ para todo $j \geq i$. Estes dois fatos implicam que a sequência de cadeias $\left\{\mathbf{D}^{(n)}\right\}_{n \in \mathbb{Z}}$ verifica

$$
D_{i}^{(n)}=0 \Rightarrow D_{k}^{(n)}=D_{k}^{(m)}, \quad \forall n \leq i \leq m \leq k .
$$

Utilizando primeiro (6.18), e depois (6.17), obtemos para $l \geq \sigma-1$

$$
\bigcap_{i=-l-1}^{-\sigma} \bigcup_{k=i+1}^{n}\left\{D_{k}^{(i)}=0\right\}=\bigcap_{i=-l-1}^{-\sigma} \bigcup_{k=-\sigma+1}^{n}\left\{D_{k}^{(i)}=0\right\}=\bigcup_{k=-\sigma+1}^{n}\left\{D_{k}^{(-l-1)}=0\right\}
$$

Segue pelos Lemas 6.2 e 6.4 que para todo $l \geq \sigma-1$ e $n \geq 0$

$$
\{\bar{\theta}[0, n]<-l\} \subset \bigcup_{k=-\sigma+1}^{n}\left\{D_{k}^{(-l-1)}=0\right\}
$$

Portanto

$$
\mathbb{P}(\bar{\theta}[0, n]<-l) \leq \sum_{k=-\sigma+1}^{n} \mathbb{P}\left(D_{k}^{(-l-1)}=0\right)
$$


Utilizando a invariância por translação de $\overline{\mathbf{Z}}$, obtemos

$$
\mathbb{P}(\bar{\theta}[0, n]<-l) \leq \sum_{k=l-\sigma+2}^{l+n+1} \mathbb{P}\left(D_{k}^{(0)}=0\right)
$$

Lema 6.6. Se $(\tau, p)$ possui um bom contexto $w$ que é também $\epsilon$-regular tal que

$$
\sum_{i \geq 0}\left(1-\epsilon^{|w|}\right)^{\bar{r}(i)}<+\infty
$$

então

$$
\sum_{k \geq 1} u_{k}<+\infty
$$

Demonstração. Vamos denotar

$$
\xi:=\inf \left\{k \geq 1: D_{k}^{(0)}=0\right\}
$$

e

$$
\mathbb{P}(\xi=k)=f_{k}
$$

Com estas notações, temos para $l \geq \sigma-1$

$$
\mathbb{P}(\bar{\theta}[0, n]<-l) \leq \sum_{k=l-\sigma+2}^{l+n+1} u_{k}
$$

Lembramos que a cadeia $\mathbf{D}^{(0)}$ é uma cadeia regenerativa a valor em $\mathbb{N}$, e o símbolo regenerativo é o 0. Vamos utilizar este fato agora. Calculamos

$$
\mathbb{P}\left(D_{k}^{(0)}=0\right)=\sum_{i=1}^{k} \mathbb{P}\left(D_{k}^{(0)}=0, \xi=i\right)=\sum_{i=1}^{k} \mathbb{P}\left(D_{k}^{(0)}=0, D_{1}^{(0)} \geq 1, \ldots, D_{i-1}^{(0)} \geq 1, D_{i}^{(0)}=0\right)
$$

Utilizando (6.18):

$$
\mathbb{P}\left(D_{k}^{(0)}=0, D_{1}^{(0)} \geq 1, \ldots, D_{i-1}^{(0)} \geq 1, D_{i}^{(0)}=0\right)=\mathbb{P}\left(D_{k}^{(i)}=0, D_{1}^{(0)} \geq 1, \ldots, D_{i-1}^{(0)} \geq 1, D_{i}^{(0)}=0\right)
$$

e como os eventos $\left\{D_{k}^{(i)}=0\right\}$ e $\left\{D_{1}^{(0)} \geq 1, \ldots, D_{i-1}^{(0)} \geq 1, D_{i}^{(0)}=0\right\}$ são disjuntos (para $k \geq i+1$, 
$\left\{D_{k}^{(i)}=0\right\}$ é $\mathcal{F}\left(U_{i+1}^{k}\right)$-mensurável) então

$$
\mathbb{P}\left(D_{k}^{(0)}=0, \xi=i\right)=\mathbb{P}\left(D_{k}^{(i)}=0\right) \mathbb{P}\left(D_{1}^{(0)} \geq 1, \ldots, D_{i-1}^{(0)} \geq 1, D_{i}^{(0)}=0\right)=\mathbb{P}\left(D_{k}^{(i)}=0\right) \mathbb{P}(\xi=i)
$$

e por invariância por translação, temos $\mathbb{P}\left(D_{k}^{(i)}=0\right)=\mathbb{P}\left(D_{k-i}^{(0)}=0\right)=u_{k-i}$. Utilizando a convenção $f_{0}:=0$ e $u_{0}=1$, acabamos de provar que

$$
u_{k}=\sum_{i=1}^{k} f_{i} u_{k-i} .
$$

Sabe-se (ver por exemplo Feller (1968, chap. XIII.10) Theorem.1) que quando duas sequências de números reais positivos estão ligadas por uma relação como (6.20), então

$$
\sum_{i \geq 1} f_{i}<1 \Rightarrow \sum_{n \geq 1} u_{n}<+\infty
$$

A cadeia $\mathbf{D}^{(0)}$ toca zero pela primeira vez somente a certos tempos: $\xi=k$ se e somente se $k$ pertence ao seguinte conjunto de índices (ver Figura 6.3)

$$
\{1,2, \ldots, \sigma\} \cup \bigcup_{i \geq 0}\{\sigma+i+\bar{r}(\sigma-1+i)\}
$$

Este fato está ilustrado na Figura 6.3. Nesta figura, podemos ver como o evento $\{\xi=\sigma+i+\bar{r}(\sigma-$ $1+i)\}$ pode ocorrer.

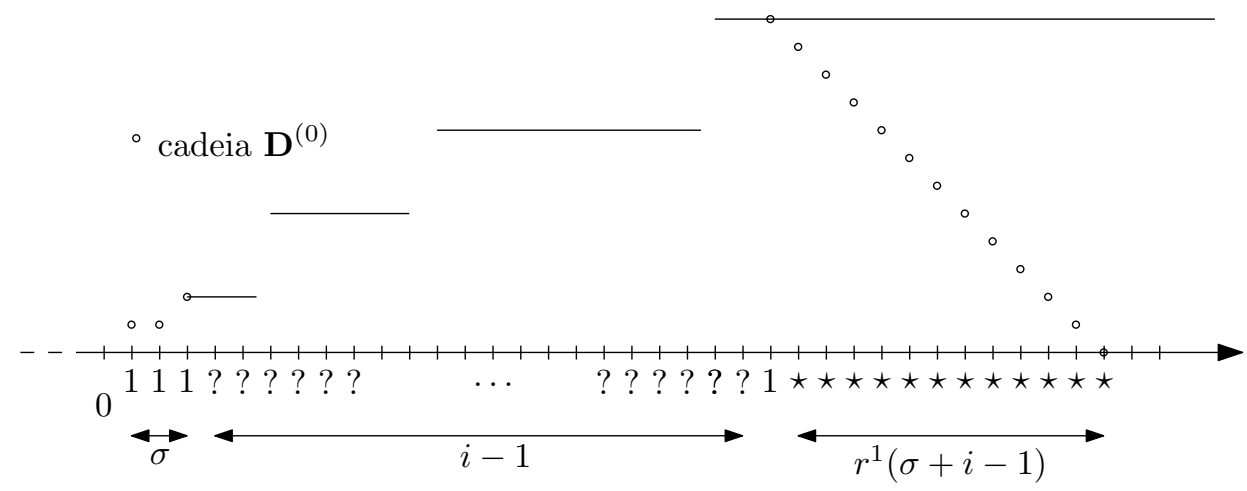

Figura 6.3: Ilustração da equação (6.21). A sequência de "?" significa que pode ocorrer qualquer coisa nestes tempos, desde que a cadeia $\mathbf{D}^{(0)}$ não toca 0. 
Além disso, pela Definição 6.15 da cadeia $\mathbf{D}^{(0)}$ temos

$$
\{\xi=k\}=\left\{\bar{Z}_{1}^{k-1}=1^{k-1}\right\} \cap\left\{\bar{Z}_{k} \neq 1\right\} \quad \text { para } \quad k=1, \ldots, \sigma
$$

e

$$
\bigcup_{k \geq \sigma+1}\{\xi=k\} \subset\left\{\bar{Z}_{1}^{\sigma}=1^{\sigma}\right\} \cap \bigcup_{i \geq 0}\left\{1 \notin \bar{Z}_{i+\sigma}^{i+\sigma+\bar{r}(\sigma-1+i)}\right\} .
$$

(este fato é ilustrado na Figura 6.3). Portanto temos

$$
f_{1}=1-\epsilon^{|w|}, f_{2}=\epsilon^{|w|}\left(1-\epsilon^{|w|}\right), \ldots, f_{\sigma}=\epsilon^{(\sigma-1)|w|}\left(1-\epsilon^{|w|}\right)
$$

e para $k \geq \sigma+1$, temos

$$
\sum_{k \geq \sigma+1} f_{k} \leq \epsilon^{\sigma|w|} \sum_{i \geq 0}\left(1-\epsilon^{|w|}\right)^{\bar{r}(\sigma-1+i)}
$$

$\log 0$

$$
\sum_{k \geq 1} f_{k}=\left(1-\epsilon^{|w|}\right) \sum_{k=1}^{\sigma} \epsilon^{(k-1)|w|}+\epsilon^{\sigma|w|} \sum_{i \geq \sigma-1}\left(1-\epsilon^{|w|}\right)^{\bar{r}(i)} .
$$

Para mostrar que $\sum_{k \geq 1} f_{k}<1$, basta mostrar que $\sum_{i \geq \sigma-1}\left(1-\epsilon^{|w|}\right)^{\bar{r}(i)}<1$. Nas condições deste lema, sabemos que $\sum_{i \geq 0}\left(1-\epsilon^{|w|}\right)^{\bar{r}(i)}<+\infty$, portanto, pegando $\sigma$ suficientemente grande obtemos a seguinte sequência de implicações:

$$
\begin{aligned}
\sum_{i \geq 0}\left(1-\epsilon^{|w|}\right)^{\bar{r}(i)}<+\infty & \Rightarrow \sum_{i \geq \sigma-1}\left(1-\epsilon^{|w|}\right)^{\bar{r}(i)}<1 \Rightarrow \sum_{k \geq 1} f_{k}<1 \\
& \Rightarrow \sum_{k \geq 1} u_{k}<+\infty
\end{aligned}
$$

\subsection{Prova do teorema}

A prova do teorema é simples agora.

Prova do Teorema 5.2. Estamos interessados em mostrar que $\mathbb{P}(\theta[0, n]<-l)$ converge para 0 para 
todo $-\infty<n \leq+\infty$. Temos pelos Lemas 6.1 e 6.5

$$
\mathbb{P}(\theta[0, n|w|]<-l|w|) \leq \mathbb{P}(\bar{\theta}[0, n]<-l+1) \leq \sum_{k=l-\sigma+1}^{l+n} u_{k}
$$

para todo $l \geq \sigma$. E como

$$
\mathbb{P}(\theta[0, n]<-l) \leq \mathbb{P}\left(\theta\left[0,|w|\left[\frac{n}{|w|}\right]\right]<-|w|\left\lfloor\frac{l}{|w|}\right\rfloor\right)
$$

segue que para todo $l$ tal que $\left\lfloor\frac{l}{|w|}\right\rfloor \geq \sigma$

$$
\mathbb{P}(\theta[0, n]<-l) \leq \sum_{k=\left\lfloor\frac{l}{|w|}\right\rfloor-\sigma+1}^{\left\lfloor\frac{l}{|w|}\right\rfloor+\left\lceil\frac{n}{|w|}\right\rceil} u_{k}
$$

Resta provar que nas condições do Teorema 5.2, a sequência $\left\{u_{k}\right\}_{k \geq 1}$ é somável. Nas condições do Teorema 5.2, temos

$$
\limsup _{k \rightarrow \infty} \frac{\log \ell^{w}(k)}{\log \left(1 /\left(1-\epsilon^{|w|}\right)\right)^{k /|w|}}<1
$$

portanto, existe um número inteiro positivo $\bar{k}$ e um número real positivo $\alpha$ tais que para todo $k$ maior que $\bar{k}$

$$
\ell^{w}(k) \leq\left(\frac{1}{1-\epsilon^{|w|}}\right)^{k /[|w|(1+\alpha)]} .
$$

Separamos dois casos.

- Quando $|w|=1$, é claro que

$$
\bar{\ell}:=\left\lceil\frac{\ell^{w}(k|w|)}{|w|}\right\rceil=\ell^{w}(k)
$$

e portanto, $\bar{\ell}(k) \leq\left(\frac{1}{1-\epsilon^{|w|}}\right)^{k /(1+\alpha)}$ para todo $k$ suficientemente grande.

- Quando $|w| \geq 2$, temos sempre que $\ell^{w}(k|w|) \geq 2$

$$
\left\lceil\frac{\ell^{w}(k|w|)}{|w|}\right\rceil \leq \ell^{w}(k|w|)
$$


Logo, para $k$ suficientemente grande

$$
\bar{\ell}:=\left\lceil\frac{\ell^{w}(k|w|)}{|w|}\right\rceil \leq\left(\frac{1}{1-\epsilon^{|w|}}\right)^{k /(1+\alpha)} .
$$

Concluímos que para $k$ suficientemente grande, existe um número real $\alpha>0$ tal que

$$
\bar{\ell}(k) \leq\left(\frac{1}{1-\epsilon^{|w|}}\right)^{k /(1+\alpha)}
$$

Pela Definição $\bar{r}$ (ver (6.9)),

$$
\bar{r}(n) \geq \frac{1+\alpha}{\log \frac{1}{1-\epsilon|w|}} \log n
$$

para todo $n$ maior que $\bar{n}$ onde $\bar{n}$ é tal que $\bar{r}(\bar{n}) \geq \bar{k}$. Portanto

$$
\sum_{n \geq 0}\left(1-\epsilon^{|w|}\right)^{\bar{r}(n)} \leq \sum_{n=0}^{\bar{n}-1}\left(1-\epsilon^{|w|}\right)^{\bar{r}(n)}+\sum_{n \geq \bar{n}} n^{-1-\alpha}
$$

é finito porque $\alpha$ é positiva. Segue que nas condições do Teorema 5.2

$$
\sum_{n \geq 0}\left(1-\epsilon^{|w|}\right)^{\bar{r}(n)}<\infty
$$

Pelo Lema 6.6, segue que $\left\{u_{k}\right\}_{k \geq 1}$ é somável. Em particular, isto implica utilizando (6.22) que para todo $l$ tal que $\left\lfloor\frac{l}{|w|}\right\rfloor \geq \sigma$

$$
\mathbb{P}(\theta[0, n]<-l) \leq \sum_{k=\left\lfloor\frac{l}{|w|}\right\rfloor-\sigma+1}^{\left\lfloor\frac{l}{|w|}\right\rfloor+\left\lceil\frac{n}{|w|}\right\rceil} u_{k} \stackrel{l \rightarrow+\infty}{\longrightarrow} 0 .
$$

Isso conclui a prova do Teorema 5.2. 


\section{Capítulo 7}

\section{Prova do Teorema 5.3}

A existência do esquema regenerativo é garantida (utilizando o Teorema 2.14) se provarmos que $\mathbb{P}(\theta[0,+\infty]=0)>0$. Pelo Lema 6.1, temos

$$
\mathcal{L}\left(U_{|w|(\bar{\theta}[0,+\infty]-1)+1}^{+\infty}\right)=1
$$

e portanto, se $\bar{\theta}[0,+\infty]=-i, i \geq \sigma-1$

$$
\theta[|w|(-i-1)+1,+\infty]=|w|(-i-1)+1
$$

Portanto, pela invariância por translação de $\mathbf{U}$

$$
\mathbb{P}(\theta[0,+\infty]=0)=\mathbb{P}(\theta[|w|(-i-1)+1,+\infty]=|w|(-i-1)+1) \geq \mathbb{P}(\bar{\theta}[0, n]=-i)
$$

Vamos fazer a conta com $i=\sigma-1$. Lembramos que $\bar{\theta}[0, n] \leq-\sigma+1$, portanto

$$
\mathbb{P}(\theta[0,+\infty]=0) \geq \mathbb{P}(\bar{\theta}[0, n]=-\sigma+1)=1-\mathbb{P}(\bar{\theta}[0, n]<-\sigma+1) .
$$

Lembramos que tinhamos a seguinte relação (equação (6.19) na prova do Lema 6.6) para $\bar{\theta}[0,+\infty]$

$$
\{\bar{\theta}[0,+\infty]<-l\}=\bigcup_{k \geq-\sigma+1}\left\{D_{k}^{(-l-1)}=0\right\}
$$


Então

$$
\mathbb{P}(\bar{\theta}[0,+\infty]<-\sigma+1)=\mathbb{P}\left(\bigcup_{k \geq 1}\left\{D_{k}^{(0)}=0\right\}\right) .
$$

O evento $\bigcup_{k \geq 1}\left\{D_{k}^{(0)}=0\right\}$ está incluído no evento $\bigcup_{k \geq 1}\{\xi=k\}$ (em que lembramos que $\xi$ é o primeiro tempo superior ou igual a 1 tal que a cadeia $\mathbf{D}^{(0)}$ toca 0$)$. Logo

$$
\mathbb{P}(\bar{\theta}[0,+\infty]<-\sigma+1) \leq \mathbb{P}\left(\bigcup_{k \geq 1}\{\xi=k\}\right)
$$

que é igual a $\sum_{k \geq 1} f_{k}$. Sabemos que nas condições do Teorema 5.2,

$$
\sum_{k \geq 1} f_{k}<1
$$

Portanto,

$$
\mathbb{P}(\theta[0,+\infty]=0) \geq 1-\sum_{k \geq 1} f_{k}>0 .
$$

Concluímos que a cadeia $\mathbf{X}(\mathbf{U})$ tem um esquema regenerativo. Para provar que o tempo médio entre dois 1's consecutivos na cadeia $\mathbf{T}$ é finito, calculamos $\mathbb{P}\left(T_{l+1}-T_{l} \geq m\right)$. Observamos que

$$
\mathbb{P}\left(T_{l+1}-T_{l} \geq m\right)=\mathbb{P}\left(\theta\left[T_{l+1}-1,+\infty\right] \leq T_{l+1}-m+1 \mid \theta\left[T_{l+1},+\infty\right]=T_{l+1}\right)
$$

e por estacionariedade

$$
\mathbb{P}\left(T_{l+1}-T_{l} \geq m\right)=\mathbb{P}(\theta[0,+\infty] \leq-m+2 \mid \theta[1,+\infty]=1) .
$$

Também, pela Definição 2.9 de $\theta[m, n]$

$$
\mathbb{P}(\theta[0,+\infty]<-m+2 \mid \theta[1,+\infty]=1)=\mathbb{P}(\theta[0]<-m+2) .
$$

Portanto, pelo Lema 6.1 temos $\mathbb{P}\left(T_{l+1}-T_{l} \geq m\right) \leq \mathbb{P}\left(\bar{\theta}[0]<-\left\lfloor\frac{(m-2)}{|w|}\right\rfloor+1\right)$. Utilizando Lema 
6.5 temos para todo $m$ tal que $\left\lfloor\frac{m-2}{|w|}\right\rfloor \geq \sigma$

$$
\mathbb{P}\left(T_{l+1}-T_{l} \geq m\right) \leq \sum_{k=\left\lfloor\frac{(m-2)}{|w|}\right\rfloor-\sigma+1}^{\left\lfloor\frac{(m-2)}{|w|}\right\rfloor} u_{k}
$$

O menor $m$ tal que $\left\lfloor\frac{m-2}{|w|}\right\rfloor \geq \sigma$ é $\sigma|w|+2$. Portanto,

$$
\begin{gathered}
\sum_{m \geq 1} \mathbb{P}\left(T_{l+1}-T_{l} \geq m\right)=\sum_{m=1}^{\sigma|w|+1} \mathbb{P}\left(T_{l+1}-T_{l} \geq m\right)+\sum_{m \geq \sigma|w|+2} \mathbb{P}\left(T_{l+1}-T_{l} \geq m\right) \\
\leq \sum_{m=1}^{\sigma|w|+1} \mathbb{P}\left(T_{l+1}-T_{l} \geq m\right)+|w| \sum_{m \geq 0} \sum_{k=m+1}^{m+\sigma} u_{k} . \\
\leq \sum_{m=1}^{\sigma|w|+1} \mathbb{P}\left(T_{l+1}-T_{l} \geq m\right)+\sigma|w| \sum_{k \geq 1} u_{k} .
\end{gathered}
$$

Este último termo é finito, porque nas condições do Teorema 5.2, a sequência $\left\{u_{k}\right\}_{k \geq 1}$ é somável. O resultado de perda de memória segue simplesmente do fato que pelo Teorema 2.13, ela é majorada pela velocidade na qual $\mathbb{P}(\theta[0]<-i)$ converge para 0 . Pelo que acabamos de mostrar acima,

$$
\sum_{i \geq 0} \mathbb{P}(\theta[0]<-i)<+\infty
$$




\section{Capítulo 8}

\section{Prova do Teorema 5.4}

Seja $(\tau, p)$ uma árvore probabilística com bom contexto $w$ verificando as condições do Teorema 5.2. Definimos a função inversa de $h^{w}$ :

$$
g^{w}(i)=\min \left\{k \geq 0: h^{w}(k)>i\right\}
$$

Denotamos por $\mathbf{X}$ a única cadeia compatível com $(\tau, p)$. Construimos uma versão acoplada $\left(\tilde{\mathbf{X}}, \tilde{\mathbf{X}}{ }^{[k]}\right)$ de $\mathbf{X}$ com sua aproximação markoviana canônica $\mathbf{X}^{[k]}$ utilizando $\mathbf{U}$ : construímos as duas juntamente com o algoritmo de simulação perfeita.

Prova do Teorema 5.4. Para todo $k$, podemos construir as duas cadeias $\tilde{\mathbf{X}}$ e $\tilde{\mathbf{X}}^{[k]}$ utilizando $\mathbf{U}$, com o algoritmo da Seção 2.5. Segue que o tempo $\theta[0]$ a partir do qual podemos reconstruir $\tilde{X}_{0}$ é também um tempo a partir do qual podemos reconstruir $\tilde{X}_{0}^{[k]}$. Para obter $\tilde{X}_{0} \neq \tilde{X}_{0}^{[k]}$, é necessário que tenha aparecido uma sequência de tamanho superior ou igual a $g^{w}(k)$ sem nenhuma ocorrência de $w$. Isso porque somente neste caso pode haver um tempo $i, \theta[0]+1 \leq i \leq 0$ tal que a construção de $\tilde{X}_{i}$ envolva um passado maior que a construção de $\tilde{X}_{i}^{[k]}$. Dado uma sequência de símbolos de $A \cup \star$, definimos

$$
R^{\bar{w}}\left(a_{m}^{n}\right):=\max \left\{i \geq 0: \text { existe } k \in \mathbb{Z}, m \leq k \leq n-i+1 \text { tal que } w \notin a_{k}^{k+i-1}\right\}
$$

que é o maior bloco sem ocorrências de $w$ na sequência $a_{m}^{n}$. Pelo que discutimos acima, temos

$$
\mathbb{P}\left(\tilde{X}_{0}^{[k]} \neq \tilde{X}_{0}\right)=\mathbb{P}\left(\tilde{X}_{0}^{[k]} \neq \tilde{X}_{0}, R^{\bar{w}}\left(\tilde{X}_{\theta[0]}^{0}\right) \geq g^{w}(k)\right)+\mathbb{P}\left(\tilde{X}_{0}^{[k]} \neq \tilde{X}_{0}, R^{\bar{w}}\left(\tilde{X}_{\theta[0]}^{0}\right)<g^{w}(k)\right)
$$




$$
=\mathbb{P}\left(\tilde{X}_{0}^{[k]} \neq \tilde{X}_{0}, R^{\bar{w}}\left(\tilde{X}_{\theta[0]}^{0}\right) \geq g^{w}(k)\right) \leq \mathbb{P}\left(R^{\bar{w}}\left(\tilde{X}_{\theta[0]}^{0}\right) \geq g^{w}(k)\right)
$$

porque $\tilde{X}_{0}^{[k]}$ não pode ser diferente de $\tilde{X}_{0}$ se não há bloco maior que $g^{w}(k)$ sem ocorrência de $w$ 's dentro de $\tilde{X}_{\theta[0]}^{0}$. Utilizamos como na prova do Teorema 5.2 , a cadeia $\overline{\mathbf{Z}}$ e a variável $\bar{\theta}[0]$. Lembramos que (ver Lema 6.1) temos

$$
\theta[0] \geq|w|(\bar{\theta}[0]-1)+1
$$

$\log 0$

$$
R^{\bar{w}}\left(\tilde{X}_{\theta[0]}^{0}\right) \leq R^{\bar{w}}\left(\tilde{X}_{|w|(\bar{\theta}[0]-1)+1}^{0}\right)
$$

e definindo agora $R^{\star}\left(\bar{Z}_{m}^{n}\right)$ como sendo o maior bloco de $\star$ 's dentro de $\bar{Z}_{m}^{n}$

$$
R^{\bar{w}}\left(\tilde{X}_{|w|(\bar{\theta}[0]-1)+1}^{0}\right) \leq R^{\star}\left(\bar{Z}_{\bar{\theta}[0]}^{0}\right)|w| .
$$

Vamos agora voltar à conta que nos interessa. Pelo que acabamos de mostrar, temos

$$
\mathbb{P}\left(\tilde{X}_{0}^{[k]} \neq \tilde{X}_{0}\right) \leq \mathbb{P}\left(R^{\star}\left(\bar{Z}_{\bar{\theta}[0]}^{0}\right)|w| \geq g^{w}(k)\right)
$$

Agora, podemos re-escrever da seguinte maneira

$$
\begin{gathered}
\mathbb{P}\left(R^{\star}\left(\bar{Z}_{\bar{\theta}[0]}^{0}\right)|w| \geq g^{w}(k)\right)=\mathbb{P}\left(R^{\star}\left(\bar{Z}_{\bar{\theta}[0]}^{0}\right)|w| \geq g^{w}(k), \bar{\theta}[0]<-f(k)\right)+ \\
\mathbb{P}\left(R^{\star}\left(\bar{Z}_{\bar{\theta}[0]}^{0}\right)|w| \geq g^{w}(k), \bar{\theta}[0] \geq-f(k)\right)
\end{gathered}
$$

em que $f$ é uma função a valor em $\mathbb{N}$ que podemos escolher.

- O primeiro termo de (8.2) é limitado por cima, isto é,

$$
\mathbb{P}\left(R^{\star}\left(\bar{Z}_{\bar{\theta}[0]}^{0}\right)|w| \geq g^{w}(k), \bar{\theta}[0]<-f(k)\right) \leq \mathbb{P}(\bar{\theta}[0]<-f(k)) \leq \sum_{j=f(k)-\sigma+2}^{f(k)+1} u_{j}
$$

pelo Lema 6.5. 
- O segundo termo de (8.2) pode ser majorado da seguinte maneira:

$$
\begin{gathered}
\mathbb{P}\left(R^{\star}\left(\bar{Z}_{\bar{\theta}[0]}^{0}\right)|w| \geq g^{w}(k), \bar{\theta}[0] \geq-f(k)\right) \leq \mathbb{P}\left(R^{\star}\left(\bar{Z}_{-f(k)}^{0}\right)|w| \geq g^{w}(k)\right) \\
\leq \mathbb{P}\left(R^{\star}\left(\bar{Z}_{-f(k)}^{0}\right) \geq\left\lfloor\frac{g^{w}(k)}{|w|}\right\rfloor\right)
\end{gathered}
$$

que é a probabilidade que exista um bloco de tamanho maior ou igual a $\left\lfloor\frac{g^{w}(k)}{|w|}\right\rfloor$ de estrelas numa sequência i.i.d., em que as estrelas aparecem com probabilidade $1-\epsilon^{|w|}$. Para estudar este termo, utilizamos os seguinte fato:

$$
\left\{R^{\star}\left(\bar{Z}_{1}^{j}\right) \geq i\right\} \subset \bigcup_{k=1}^{j-i+1}\left\{\bar{Z}_{k}^{k+i-1}=0^{i}\right\}
$$

segue a cota seguinte

$$
\begin{gathered}
\mathbb{P}\left(R^{\star}\left(\bar{Z}_{1}^{j}\right) \geq i\right) \leq(j-i+1)\left(1-\epsilon^{|w|}\right)^{i} \\
\leq j\left(1-\epsilon^{|w|}\right)^{i}
\end{gathered}
$$

para todo $i \geq 1$. Portanto, temos a seguinte cota para os segundo termo:

$$
\mathbb{P}\left(R^{\star}\left(\bar{Z}_{\bar{\theta}[0]}^{0}\right)|w| \geq g^{w}(k), \bar{\theta}[0] \geq-f(k)\right) \leq f(k)\left(1-\epsilon^{|w|}\right)^{\left\lfloor\frac{g^{w}(k)}{|w|}\right\rfloor} .
$$

Segue que para $k$ suficientemente grande

$$
\mathbb{P}\left(\tilde{X}_{0}^{[k]} \neq \tilde{X}_{0}\right) \leq \sum_{j=f(k)-\sigma+2}^{f(k)+1} u_{j}+f(k)\left(1-\epsilon^{|w|}\right)^{\left\lfloor\frac{g^{w}(k)}{|w|}\right\rfloor} .
$$

Pela Definição $9.9, g^{w}$ é uma função crescente que verifica

$$
k<g^{w}(k)+|w|+\ell^{w}\left(g^{w}(k)\right)
$$

e sabemos que nas condições do Teorema 5.2

$$
\ell^{w}(k) \leq\left(\frac{1}{1-\epsilon^{|w|}}\right)^{k /[|w|(1+\alpha)]}
$$


segue que pegando $0<\alpha^{\prime}<\alpha$, temos para $k$ suficientemente grande

$$
k<\left(\frac{1}{1-\epsilon^{|w|}}\right)^{g^{w}(k) /\left[|w|\left(1+\alpha^{\prime}\right)\right]} .
$$

Logo

$$
\left(1-\epsilon^{|w|}\right)^{\frac{g^{w}(k)}{|w|}} \leq k^{-1-\alpha^{\prime}}
$$

A desigualdade (8.3) torna-se

$$
\mathbb{P}\left(\tilde{X}_{0}^{[k]} \neq \tilde{X}_{0}\right) \leq \sum_{j=f(k)-\sigma+2}^{f(k)+1} u_{j}+f(k)\left(1-\epsilon^{|w|}\right)^{-1} k^{-1-\alpha^{\prime}}
$$

que vai para zero escolhendo-se bem a função $f$, pelo Lema 6.6 e o fato que $\alpha^{\prime}>0$. 


\section{Capítulo 9}

\section{Prova do Teorema 5.5}

Seja $(\tau, p)$ uma árvore probabilística verificando as condições do Teorema 5.5, tendo como bom contexto o símbolo $b$, elemento de $A$. Consideramos a árvore $\left.\tau\right|_{b}$ obtida truncando todos os contextos $v$ de $\tau$ na altura $m^{b}(v)+1$ (os contextos $v$ tais que $m^{b}(v)=+\infty$ não estão truncados). Alguns exemplos são:

- se $\tau$ é a árvore da Figura 2.3, então $\left.\tau\right|_{1}$ é a árvore esparsa (Figura 2.1(b)),

- se $\tau$ é a árvore da Figura 5.3, então $\left.\tau\right|_{1}$ é a árvore da Figura 4.2.

Chamamos a atenção sobre a diferença entre este truncamento e o truncamento na altura $k$ utilizado na seção anterior. É claro que $\left.\tau\right|_{b}$ é sempre uma árvore tendo $b$ como contexto terminal. Em particular, observamos que para todo $i \geq 1$ o conjunto $\mathcal{I}_{i}^{b}$ é o mesmo para $\tau$ e para $\left.\tau\right|_{b}$. Definimos para todo $i \geq 1, s \in \mathcal{I}_{i}^{b}$

$$
p_{i}(b \mid s b):=\inf \left\{p(b \mid s b c): c \in \mathcal{C}_{i}^{b}(s)\right\}
$$

e

$$
p_{i}(b)=\inf \left\{p_{i}(b \mid s b): s \in \mathcal{I}_{i}^{b}\right\}
$$

Consideramos a árvore probabilística $\left(\left.\tau\right|_{b},\left.p\right|_{b}\right)$ onde $\left.\tau\right|_{b}$ é definida acima e $\left.p\right|_{b}$ é uma família de probabilidades de transição definida da seguinte maneira. Para todo $a \in A$ e todo $v \in \tau_{\bar{b}}$ colocamos

$$
\left.p\right|_{b}(a \mid v)=p(a \mid v)
$$

para todo $i \geq 0$,

$$
\left.p\right|_{b}(b \mid s b)=p_{i}(b), \quad \forall s \in \mathcal{I}_{i}^{b}
$$


e para todo $a \in A_{\bar{b}}$ e todo $i \geq 0$ tomamos

$$
\left.p\right|_{b}(a \mid s b)=g_{i}(a), \quad \forall s \in \mathcal{I}_{i}^{b}
$$

em que para todo $i, g(i)$ é uma função $A_{\bar{b}} \rightarrow[0,1]$ e verifica

$$
\sum_{a \in A_{\bar{b}}} g_{i}(a)=1-p_{i}(b)
$$

Sabemos pela Proposição 4.1 que existe uma única cadeia estacionária compatível com $\left(\left.\tau\right|_{b},\left.p\right|_{b}\right)$ se

$$
\sum_{i \geq 1} \prod_{j=0}^{i-1}\left(1-p_{j}(b)\right)<+\infty .
$$

Chamamos $\mathbf{Y}$ esta cadeia e $\mathbb{Q}$ sua lei.

Lembramos que na prova do Teorema 5.2, utilizamos uma cadeia $\overline{\mathbf{Z}}$ a valor em $\{1, \star\}$ que é construída com $\mathbf{U}$. Também definimos uma cadeia $\overline{\mathbf{Z}}$, mas dessa vez utilizando a cadeia $\mathbf{Y}$, ou seja, para todo $m \in \mathbb{Z}$ colocamos $\bar{Z}_{m}=1$ quando $Y_{m}=b$, e $\bar{Z}_{m}=\star$ no caso contrário. A diferença com a prova anterior é que $\overline{\mathbf{Z}}$ não é mais uma cadeia i.i.d. Se trata da cadeia esparsa sobre $\{1, \star\}$ : para todo $m \in \mathbb{Z}$ e todo $l \geq 0$

$$
\mathbb{Q}\left(\bar{Z}_{m}=1 \mid \bar{Z}_{m-1} \neq 1, \ldots, \bar{Z}_{m-l} \neq 1, \bar{Z}_{m-l-1}=1\right)=p_{l}(b) .
$$

Também definimos $\bar{\theta}[0, n]$ como antes, ou seja, como (6.8) com a única diferença que agora a cadeia $\overline{\mathbf{Z}}$ não tem as mesmas propriedades. Assim

$$
\begin{aligned}
\bar{\theta}[0, n]:=\max \{k \leq m-\sigma+1: \\
\left.\bar{Z}_{k}^{k+\sigma-1}=1^{\sigma} \text { e } \bar{h}(j-\bar{t}(j)) \leq j-k+1, j=k+\sigma, \ldots, n\right\} .
\end{aligned}
$$

Portanto, a cadeia $\overline{\mathbf{Z}}$ e a variável $\bar{\theta}$ têm o mesmo papel que a cadeia $\overline{\mathbf{Z}}$ e a variável $\bar{\theta}$ na Seção 6.2. Lembramos que na Seção 6.2, a hipótese que $\overline{\mathbf{Z}}$ era i.i.d. só foi utilizada a partir do Lema 6.6. Portanto, podemos prosseguir direitamente até este lema: temos para todo $i \in \mathbb{Z}$ uma cadeia $\mathbf{D}^{(i)}$ 
definida da seguinte maneira. Para $j \leq i, D_{j}^{(i)}=0$, e para $j \geq i+1$

$$
D_{j}^{(i)}=\left\{\begin{array}{ccccc}
0 & \text { se } & \bar{Z}_{j} \neq 1 & \text { e } & j<j^{\prime}+1 \\
c-1 & \text { se } & \bar{Z}_{j} \neq 1 & \text { e } & j \geq j^{\prime}+\sigma \\
1 & \text { se } & \bar{Z}_{j}=1 & \text { e } & j<j^{\prime}+1 \\
\bar{r}\left(\left(j-j^{\prime}-1\right)|w|\right) & \text { se } & \bar{Z}_{j}=1 & \text { e } & j \geq j^{\prime}+\sigma
\end{array}\right.
$$

em que, $1 \leq c=D_{j-1}^{(i)}$ e lembramos que a cadeia $\mathbf{D}^{(i)}$ é tal que

$$
\{\bar{\theta}[0,+\infty]<-l\} \subset \bigcup_{j \geq-\sigma+1}\left\{D_{j}^{(-l-1)}=0\right\}
$$

Estamos agora na mesma situação daquela que antecede imediatamente o Lema 6.6, com a diferença que a cadeia $\overline{\mathbf{Z}}$ é a cadeia esparsa como já dizemos.

O principal problema é que a dependência da cadeia $\overline{\mathbf{Z}}$ faz com que o evento $\left\{D_{j}^{(i)}=0\right\}$ não seja regenerativo para $\mathbf{D}^{(0)}$. Portanto, temos que considerar um outro evento, relevante para o nosso problema.

Lema 9.1. Para $l \geq \sigma$

$$
\mathbb{Q}(\bar{\theta}[0,+\infty]<-l) \leq \sum_{j \geq l-\sigma+2} \mathbb{Q}\left(D_{j}^{(0)}=0, D_{j+1}^{(0)}=1\right)
$$

Demonstração. Começamos com a desisgualdade (escrevemos $\bar{\theta}:=\bar{\theta}[0,+\infty]$ )

$$
\{\bar{\theta}<-l\} \subset \bigcup_{j \geq-\sigma+1}\left\{D_{j}^{(-l-1)}=0\right\}
$$

Pela definição da cadeia $\mathbf{D}^{(-l-1)}$, temos para $j \geq-\sigma+1$

$$
\left\{D_{j}^{(-l-1)}=0\right\}=\left\{D_{i}^{(-l-1)}=0, i \geq j\right\} \cup \bigcup_{i \geq j}\left\{D_{j}^{(-l-1)}=\ldots=D_{i}^{(-l-1)}=0, D_{i+1}^{(-l-1)}=1\right\},
$$

isto é, particionamos em relação à primeira ocorrência do estado 1 após $j$. Mas podemos usar a 
seguinte inclusão para todo $i \geq j$

$$
\left\{D_{j}^{(-l-1)}=\ldots=D_{i}^{(-l-1)}=0, D_{i+1}^{(-l-1)}=1\right\} \subset\left\{D_{i}^{(-l-1)}=0, D_{i+1}^{(-l-1)}=1\right\} .
$$

Chegamos então a

$$
\begin{gathered}
\bigcup_{j \geq-\sigma+1}\left\{D_{j}^{(-l-1)}=0\right\} \subset \bigcup_{j \geq-\sigma+1}\left\{D_{i}^{(-l-1)}=0, i \geq j\right\} \cup \bigcup_{i \geq j}\left\{D_{i}^{(-l-1)}=0, D_{i+1}^{(-l-1)}=1\right\} \\
=\bigcup_{j \geq-\sigma+1}\left\{D_{i}^{(-l-1)}=0, i \geq j\right\} \cup\left\{D_{j}^{(-l-1)}=0, D_{j+1}^{(-l-1)}=1\right\} .
\end{gathered}
$$

Em termos de probabilidades, temos

$$
\mathbb{Q}(\bar{\theta}<-l) \leq \sum_{j \geq-\sigma+1}\left(\mathbb{Q}\left(D_{i}^{(-l-1)}=0, i \geq j\right)+\mathbb{Q}\left(D_{j}^{(-l-1)}=0, D_{j+1}^{(-l-1)}=1\right)\right)
$$

O evento $\left\{D_{i}^{(-l-1)}=0, i \geq j\right\}$ ocorre se e somente se $\left\{\bar{Z}_{i}^{+\infty}=0_{i}^{+\infty}\right\}$. Em nossas condições a sequência $\overline{\mathbf{Z}}$ tem uma infinidade de 1's (mais ainda, a distância média entre dois 1's consecutivos é finita) isso implica que $\mathbb{Q}\left(D_{i}^{(-l-1)}=0, i \geq j\right)=0$ para todo $j \geq-\sigma+1$. Logo

$$
\mathbb{Q}(\bar{\theta}<-l) \leq \sum_{j \geq-\sigma+!} \mathbb{Q}\left(D_{j}^{(-l-1)}=0, D_{j+1}^{(-l-1)}=1\right)=\sum_{j \geq l-\sigma+2} \mathbb{Q}\left(D_{j}^{(0)}=0, D_{j+1}^{(0)}=1\right)
$$

onde a última igualdade segue do fato que $\overline{\mathbf{Z}}$ é invariante por translação.

Lema 9.2. Nas condições do Teorema 5.5

$$
\mathbb{Q}(\bar{\theta}>-\infty)=1
$$

Demonstração. Utilizando o Lema 9.1, podemos ver que basta provar que nas condições do Teorema 5.5 , temos

$$
\sum_{j \geq 1} \mathbb{Q}\left(D_{j-1}^{(0)}=0, D_{j}^{(0)}=1\right)<+\infty
$$

Mais uma vez, estamos com uma cadeia $\mathbf{D}^{(0)}$ regenerativa, e a sequência regenerativa é 01. Para ver isto, basta observar que se temos $\left(D_{1}^{(0)}, \ldots, D_{n}^{(0)}\right)=a_{1}^{n}$ (lembramos que $D_{0}^{(0)}=0$ por definição) 
com 01 ocorrendo somente nos tempos $i$ e $j$ e $n, 1 \leq i<j-1<n$, então

$$
\begin{gathered}
\mathbb{Q}\left(\left(D_{1}^{(0)}, \ldots, D_{n}^{(0)}\right)=a_{1}^{n}\right) \\
=\mathbb{Q}\left(\left(D_{1}^{(0)}, \ldots, D_{i}^{(0)}\right)=a_{1}^{i},\left(D_{i}^{(i-1)}, \ldots, D_{j}^{(i-1)}\right)=1 a_{i+1}^{j},\left(D_{j}^{(j-1)}, \ldots, D_{n}^{(j-1)}\right)=1 a_{j+1}^{n}\right) .
\end{gathered}
$$

Esse último segue da relação (6.18). Além disso, como $a_{i-1} a_{i}=a_{j-1} a_{j}=01$, isto significa em particular que $\bar{Z}_{i}=\bar{Z}_{j}=1$, isto é, $i$ e $j$ são tempos de renovação para a cadeia $\overline{\mathbf{Z}}$. Portanto

$$
\begin{gathered}
\mathbb{Q}\left(\left(D_{1}^{(0)}, \ldots, D_{i}^{(0)}\right)=a_{1}^{i},\left(D_{i}^{(i-1)}, \ldots, D_{j}^{(i-1)}\right)=1 a_{i+1}^{j},\left(D_{j}^{(j-1)}, \ldots, D_{n}^{(j-1)}\right)=1 a_{j+1}^{n}\right) \\
=\mathbb{Q}\left(\left(D_{1}^{(0)}, \ldots, D_{i}^{(0)}\right)=a_{1}^{i-2} 01\right) \\
\times \mathbb{Q}\left(\left(D_{i+1}^{(i-1)}, \ldots, D_{j}^{(i-1)}\right)=a_{i+1}^{j-2} 01 \mid D_{i}^{(i-1)}=1\right) \\
\times \mathbb{Q}\left(\left(D_{j+1}^{(j-1)}, \ldots, D_{n}^{(j-1)}\right)=a_{j+1}^{n-2} 01 \mid D_{j}^{(j-1)}=1\right) .
\end{gathered}
$$

Agora, utilizando a invariância por translação da cadeia $\overline{\mathbf{Z}}$, vemos que

$$
\mathbb{Q}\left(\left(D_{i+1}^{(i-1)}, \ldots, D_{j}^{(i-1)}\right)=a_{i+1}^{j-2} 01 \mid D_{i}^{(i-1)}=1\right)=\mathbb{Q}\left(\left(D_{2}^{(0)}, \ldots, D_{j-i+1}^{(0)}\right)=a_{i+1}^{j-2} 01 \mid D_{1}^{(0)}=1\right)
$$

e

$$
\mathbb{Q}\left(\left(D_{j+1}^{(j-1)}, \ldots, D_{n}^{(j-1)}\right)=a_{j+1}^{n-2} 01 \mid D_{j}^{(j-1)}=1\right)=\mathbb{Q}\left(\left(D_{2}^{(0)}, \ldots, D_{n-j+1}^{(0)}\right)=a_{j+1}^{n-2} 01 \mid D_{1}^{(0)}=1\right) .
$$

Obsevamos a decomposição em ciclos, com a diferença que há um atraso (delay), que é o primeiro ciclo que agora não tem a mesma distribuição que os subsequentes. Utilizamos a notação $d_{1}=$ $\mathbb{Q}\left(D_{1}^{(0)}=1\right)$ e

$$
d_{i}=\mathbb{Q}\left(\left(D_{1}^{(0)}, \ldots, D_{i}^{(0)}\right)=a_{1}^{i-2} 01\right), \quad i \geq 2,01 \notin a_{1}^{i-2}
$$

que é a distribuição do atraso. A distribuição dos ciclos subsequentes é denotada como antes por

$$
f_{i}=\mathbb{Q}\left(\left(D_{2}^{(0)}, \ldots, D_{i+1}^{(0)}\right)=a_{2}^{i-1} 01 \mid D_{1}^{(0)}=1\right), \quad i \geq 2,01 \notin a_{2}^{i-1}
$$


Finalmente, denotamos

$$
v_{i}:=\mathbb{Q}\left(D_{i-1}^{(0)}=0, D_{i}^{(0)}=1\right), i \geq 1
$$

Nas notações acima, a sequência $a_{i}^{i-1}$ é a sequência vazia. Com estas notações, temos para $l \geq \sigma$

$$
\mathbb{P}(\bar{\theta}<-l) \leq \sum_{k \geq l-\sigma+1} v_{k}
$$

e lembramos que queremos mostrar que $\left\{v_{k}\right\}_{k \geq 1}$ é somável. Para isto, utilizamos a estrutura regenerativa da cadeia $\mathbf{D}^{(0)}$ : se ocorreu 01 no tempo $k$, isso pode ser devido ás duas seguintes possibilidade (1) $D_{1}^{(0)}=\ldots=D_{k-1}^{(0)}=0$ e $D_{k}^{(0)}=1$, isto ocorre com probabilidade $d_{k}$, (2) ocorreu $D_{j-1}^{(0)}=0, D_{j}^{(0)}=1$ num tempo $j$ anterior a $k$ e o primeiro tempo após o tempo $j$ em que ocorreu de novo foi o tempo $k$. Isto é podemos decompor

$$
\begin{gathered}
v_{k}=d_{k}+\sum_{j=1}^{k} \mathbb{Q}\left(D_{j-1}^{(0)}=0, D_{j}^{(0)}=1,\left(D_{j+1}^{(0)}, \ldots, D_{k}^{(0)}\right)=a_{j+1}^{k-2} 01\right) \\
=d_{k}+\sum_{j=1}^{k} \mathbb{Q}\left(D_{j-1}^{(0)}=0, D_{j}^{(0)}=1,\left(D_{j}^{(j-1)}, \ldots, D_{k}^{(j-1)}\right)=1 a_{j+1}^{k-2} 01\right) \\
=d_{k}+\sum_{j=1}^{k} \mathbb{Q}\left(D_{j-1}^{(0)}=0, D_{j}^{(0)}=1\right) \mathbb{Q}\left(\left(D_{j+1}^{(j-1)}, \ldots, D_{k}^{(j-1)}\right)=a_{j+1}^{k-2} 01 \mid D_{j}^{(j-1)}=1\right) .
\end{gathered}
$$

Pela invariância por translação de $\overline{\mathbf{Z}}$, temos

$$
\begin{gathered}
\mathbb{Q}\left(\left(D_{j+1}^{(0)}, \ldots, D_{k}^{(0)}\right)=a_{j+1}^{k-2} 01 \mid D_{j-1}^{(0)}=0, D_{j}^{(0)}=1\right) \\
=\mathbb{Q}\left(\left(D_{2}^{(0)}, \ldots, D_{k-j+1}^{(0)}\right)=a_{j+1}^{k-2} 01 \mid D_{0}^{(0)}=0, D_{1}^{(0)}=1\right)=f_{k-j} .
\end{gathered}
$$

Com as notações acima, obtivemos

$$
v_{k}=d_{k}+\sum_{j=1}^{k} v_{j} f_{k-j} .
$$


Nestas condições, sabe-se (ver Feller (1968, chap. XIII.10) Theorem.1) que

$$
\sum_{i \geq 1} f_{i}<1 \Rightarrow \sum_{i \geq 1} v_{i}<+\infty
$$

Portanto, nos resta provar que $\sum_{i \geq 1} f_{i}<1$. Denotamos por $\zeta$ o primeiro tempo após o tempo 1 que ocorre 01 , e $\xi$ o primeiro tempo após o tempo 1 que ocorre 0 . Denotamos por $\mathbb{Q}^{\star}$ a lei condicionada no fato que $D_{1}^{(0)}=1$, isto é que $\bar{Z}_{1}=1$ :

$$
\mathbb{Q}\left(\zeta=j \mid D_{1}^{0}=1\right)=: \mathbb{Q}^{\star}(\zeta=j) .
$$

Utilizamos a notação $D_{k+1}^{j}=0^{j-k-1} 1$ para $D_{k+1}^{(0)}=\ldots=D_{j-1}^{(0)}=0, D_{j}^{(0)}=1$ em que o índice $(0)$ da cadeia é subentendido. Observamos que se 01 ocorreu pela primeira vez no tempo $j$, então 0 ocorreu num tempo anterior, isto é

$$
\bigcup_{j \geq 2}\{\zeta=j\}=\bigcup_{j \geq 2}^{j-1} \bigcup_{k=1}^{j}\{\xi=k\} \cap\left\{D_{k+1}^{(0)}=\ldots=D_{j-1}^{(0)}=0, D_{j}^{(0)}=1\right\} .
$$

Re-escrevendo esta dupla união, vemos que pode ser ordenada da seguinte maneira

$$
\bigcup_{j \geq 2}^{j-1} \bigcup_{k=1}^{j}\{\xi=k\} \cap\left\{D_{k+1}^{j}=0^{j-k-1} 1\right\}=\bigcup_{j \geq 1}\{\xi=j\} \cap \bigcup_{k \geq 1}\left\{D_{j+1}^{j+k}=0^{k-1} 1\right\}
$$

portanto

$$
\mathbb{Q}^{\star}\left(\bigcup_{j \geq 2}\{\zeta=j\}\right) \leq \sum_{j \geq 1} \mathbb{Q}^{\star}\left(\{\xi=j\} \cap \bigcup_{k \geq 1}\left\{D_{j+1}^{j+k}=0^{k-1} 1\right\}\right)
$$

Queremos mostrar que o termo da direita é estritamente menor que 1 nas condições do Teorema 5.5. Escrevemos $\sum_{j \geq 1} \mathbb{Q}^{\star}\left(\{\xi=j\} \cap \bigcup_{k \geq 1}\left\{D_{j+1}^{j+k}=0^{k-1} 1\right\}\right)=$

$$
\begin{gathered}
\left.\mathbb{Q}^{\star}(01 \cup 001 \cup 0001 \cup \ldots\}\right)+ \\
\left.\mathbb{Q}^{\star}(101 \cup 1001 \cup 10001 \cup \ldots\}\right)+
\end{gathered}
$$




$$
\begin{aligned}
& \left.\mathbb{Q}^{\star}(\underbrace{1 \ldots 1}_{\sigma-1} 01 \cup \underbrace{1 \ldots 1}_{\sigma-1} 001 \cup \underbrace{1 \ldots 1}_{\sigma-1} 0001 \cup \ldots\}\right)+ \\
& \left.\mathbb{Q}^{\star}(\underbrace{1 \ldots 1}_{\sigma} \underbrace{0 \ldots 0}_{\bar{r}(\sigma-1)} 1 \cup \underbrace{1 \ldots 1}_{\sigma} \underbrace{0 \ldots 0}_{\bar{r}(\sigma-1)} 01 \cup \underbrace{1 \ldots 1}_{\sigma} \underbrace{0 \ldots 0}_{\bar{r}(\sigma-1)} 001 \cup \ldots\}\right)+ \\
& \left.\mathbb{Q}^{\star}(\underbrace{1 \ldots 1}_{\sigma} 1 \underbrace{0 \ldots 0}_{\bar{r}(\sigma)} 1 \cup \underbrace{1 \ldots 1}_{\sigma} 1 \underbrace{0 \ldots 0}_{\bar{r}(\sigma)} 01 \cup \underbrace{1 \ldots 1}_{\sigma} 1 \underbrace{0 \ldots 0}_{\bar{r}(\sigma)} 001 \cup \ldots\}\right)+ \\
& \left.\mathbb{Q}^{\star}(\underbrace{1 \ldots 1}_{\sigma} ? 1 \underbrace{0 \ldots 0}_{\bar{r}(\sigma+1)} 1 \cup \underbrace{1 \ldots 1}_{\sigma} ? 1 \underbrace{0 \ldots 0}_{\bar{r}(\sigma+1)} 01 \cup \underbrace{1 \ldots 1}_{\sigma} ? 1 \underbrace{0 \ldots 0}_{\bar{r}(\sigma+1)} 001 \cup \ldots\}\right)+ \\
& \left.\mathbb{Q}^{\star}(\underbrace{1 \ldots 1}_{\sigma} ? ? 1 \underbrace{0 \ldots 0}_{\bar{r}(\sigma+2)} 1 \cup \underbrace{1 \ldots 1}_{\sigma} ? ? ? 1 \underbrace{0 \ldots 0}_{\bar{r}(\sigma+2)} 01 \cup \underbrace{1 \ldots 1}_{\sigma} ? ? 1 \underbrace{0 \ldots 0}_{\bar{r}(\sigma+2)} 001 \cup \ldots\}\right)+
\end{aligned}
$$

Explicamos agora a longa equação acima. Os 0 e 1 se referem à cadeia $\overline{\mathbf{Z}}$. Para $j=1, \ldots, \sigma$, temos que

$$
\{\xi=j\}=\left\{\bar{Z}_{1}=\ldots=\bar{Z}_{j-1}=1, \bar{Z}_{j}=0\right\}
$$

Isso explica o primeiro elemento das uniãos de $(9.3,9.4,9.5)$. Em cada uma destas quantidades, as uniãos subsequentes correspondem às possibilidades de ter um novo 1 logo após $\xi$, ou num tempo depois. Para $j \geq \sigma+1$, a cadeia $\mathbf{D}^{(0)}$ só pode tocar zero pela primeira vez nos tempos $j$ tais que $j=\sigma+l+\bar{r}(\sigma+l-1), l \geq 0$, e para isso acontecer para um fixado $l$, a cadeia $\overline{\mathbf{Z}}$ deve ter verificado

$$
\bar{Z}_{1}=\ldots=\bar{Z}_{\sigma}=1, \bar{Z}_{\sigma+l}=1, \bar{Z}_{\sigma+l+1}=\ldots=\bar{Z}_{\sigma+l+\bar{r}(\sigma+l-1)}=0
$$

enquanto não têm restrições sobre $\bar{Z}_{\sigma+1}^{\sigma+l-1}$, quando $l \geq 2$ (isso corresponde aos "?"). Isso justifica o primeiro elemento das uniões de $(9.6,9.7,9.8,9.9)$. Para os elementos subsequentes destas uniões, a justificativa é a mesma que precedentemente. Calculamos agora, os elementos da comprida equação acima:

$$
9.3=\mathbb{Q}^{\star}(0), 9.4=\mathbb{Q}^{\star}(10), \ldots, 9.5=\mathbb{Q}^{\star}\left(1^{\sigma-1} 0\right), \ldots
$$




$$
9.6=\mathbb{Q}^{\star}\left(1^{\sigma} 0^{\bar{r}(\sigma-1)}\right), 9.7=\mathbb{Q}^{\star}\left(1^{\sigma} 10^{\bar{r}(\sigma)}\right), \quad 9.8=\mathbb{Q}^{\star}\left(1^{\sigma} ? 10^{\bar{r}(\sigma+1)}\right), \quad 9.9=\mathbb{Q}^{\star}\left(1^{\sigma} ? ? 10^{\bar{r}(\sigma+2)}\right), \ldots
$$

Após 9.9 , os termos são do tipo $\mathbb{Q}^{\star}\left(1^{\sigma} ?^{i} 10^{\bar{r}(\sigma+i)}\right)$. Lembramos que a cadeia $\overline{\mathbf{Z}}$ é a cadeia esparsa sobre $\{0,1\}$ e que para todo $l \geq 0$

$$
\mathbb{Q}\left(\bar{Z}_{m}=1 \mid \bar{Z}_{m-1} \neq 1, \ldots, \bar{Z}_{m-l} \neq 1, \bar{Z}_{m-l-1}=1\right)=p_{l}
$$

Portanto, podemos decompor $\mathbb{Q}^{\star}\left(1^{\sigma} ?^{i} 10^{\bar{r}(\sigma+i)}\right)$ em blocos da seguinte maneira:

$$
\mathbb{Q}^{\star}\left(1^{\sigma} ?^{i} 10^{\bar{r}(\sigma+i)}\right)=\mathbb{Q}^{\star}\left(1^{\sigma}\right) \mathbb{Q}^{\star}\left(?^{i} 1 \mid 1\right) \mathbb{Q}^{\star}\left(0^{\bar{r}(\sigma+i)} \mid 1\right)=\mathbb{Q}^{\star}\left(1^{\sigma}\right) \mathbb{Q}^{\star}\left(?^{i} 1 \mid 1\right) \prod_{j=0}^{\bar{r}(\sigma+i)-1}\left(1-p_{j}(b)\right)
$$

e podemos utilizar a seguinte majoração

$$
\mathbb{Q}^{\star}\left(1^{\sigma} ?^{i} 10^{\bar{r}(\sigma+i)}\right) \leq \mathbb{Q}^{\star}\left(1^{\sigma}\right) \prod_{j=0}^{\bar{r}(\sigma+i)-1}\left(1-p_{j}(b)\right)
$$

Voltando agora ao somatório que nos interessa: $\sum_{j \geq 1} \mathbb{Q}^{\star}\left(\{\xi=j\} \cap \bigcup_{k \geq 1}\left\{D_{j+1}^{j+k}=0^{k-1} 1\right\}\right) \leq$

$$
\begin{gathered}
\mathbb{Q}^{\star}(0)+\mathbb{Q}^{\star}(10)+\ldots+\mathbb{Q}^{\star}\left(1^{\sigma-1} 0\right)+\mathbb{Q}^{\star}\left(1^{\sigma}\right)\left(\prod_{j=0}^{\bar{r}(\sigma-1)-1}\left(1-p_{j}(b)\right)+\prod_{j=0}^{\bar{r}(\sigma)-1}\left(1-p_{j}(b)\right)+\ldots\right)= \\
\quad\left(1-\mathbb{Q}^{\star}\left(1^{\sigma}\right)\right)+\mathbb{Q}^{\star}\left(1^{\sigma}\right)\left(\prod_{j=0}^{\bar{r}(\sigma-1)-1}\left(1-p_{j}(b)\right)+\prod_{j=0}^{\bar{r}(\sigma)-1}\left(1-p_{j}(b)\right)+\ldots\right) .
\end{gathered}
$$

Para ter $\sum_{j \geq 1} \mathbb{Q}^{\star}\left(\{\xi=j\} \cap \bigcup_{k \geq 1}\left\{D_{j+1}^{j+k}=0^{k-1} 1\right\}\right)<1$, basta ter

$$
\prod_{j=0}^{\bar{r}(\sigma-1)-1}\left(1-p_{j}(b)\right)+\prod_{j=0}^{\bar{r}(\sigma)-1}\left(1-p_{j}(b)\right)+\ldots<1
$$

isto é

$$
\sum_{l \geq \sigma-1} \prod_{j=0}^{\bar{r}(l)-1}\left(1-p_{j}(b)\right)<1
$$

Como já observamos (observação 6.1), no caso em que $|w|=1$, temos $\ell^{w}=\ell^{b} \equiv \bar{\ell}$, portanto $r^{w}=r^{b} \equiv \bar{r}$. Nas condições do Teorema 5.5, sabemos que $\sum_{l \geq 0} \prod_{j=0}^{\bar{r}(l)-1}\left(1-p_{j}(b)\right)<+\infty$, portanto, tomando $\sigma$ suficientemente grande garante que $\sum_{l \geq \sigma-1} \prod_{j=0}^{\bar{r}(l)-1}\left(1-p_{j}(b)\right)<1$. Isso implica que 
$\sum_{j \geq 1} v_{j}<+\infty$ e conclui a prova do lema.

Prova do Teorema 5.5. Consideramos um passado infinito $a_{-\infty}^{-1}$ de símbolos em $A$ distribuído de acordo com a lei estacionária $\mathbb{Q}$ de $\mathbf{Y}$. Construímos a cadeia $\left([Y(\mathbf{U})]^{a_{-\infty}^{-1}, 0}\right)$ sobre o passado fixado $a_{-\infty}^{-1}$, utilizando uma sequência $\mathbf{U}$ de variáveis i.i.d. uniformemente distribuída em [0,1[. Como antes, chamamos $\mathbb{P}$ a lei de $\mathbf{U}$. A construção é feita com as partições $\left\{J^{\left.\tau\right|_{b}}(a \mid v)\right\}_{a \in A,\left.v \in \tau\right|_{b} \cup \emptyset}$ obtida com o método da Seção 2.4 e a árvore probabilística $\left(\left.\tau\right|_{b},\left.p\right|_{b}\right)$. Utilizando o método apresentado na Seção 2.4, temos

$$
\mathbb{P}\left(\mathbf{Y}(\mathbf{U})^{a_{-\infty}^{-1}, 0} \in \cdot\right)=\mathbb{Q}(\cdot) .
$$

Agora, construímos uma outra cadeia $\mathbf{X}(\mathbf{U})^{a_{-\infty}^{-1}, 0}$, utilizando $\mathbf{U}$, mas dessa vez com as partições $\{J(a \mid v)\}_{a \in A, v \in \tau \cup \emptyset}$ obtidas com a árvore probabilística $(\tau, p)$ de interesse nesta seção. Pelas definições das partições $\left\{J^{\left.\tau\right|_{b}}(a \mid v)\right\}_{a \in A,\left.v \in \tau\right|_{b} \cup \emptyset}$ e $\{J(a \mid v)\}_{a \in A, v \in \tau \cup \emptyset}$ e as probabilidades de transição $\left.p\right|_{b}$ (ver começo deste capítulo) temos que

$$
[Y(\mathbf{U})]_{i}^{a_{-\infty}^{-1}, 0}=b \Rightarrow(X(\mathbf{U}))_{i}^{a_{-\infty}^{-1}, 0}=b
$$

para todo $i \geq 0$. A cadeia $\mathbf{Y}(\mathbf{U})^{a_{-\infty}^{-1}, 0}$ é estacionária para todo $i \in \mathbb{Z}$. Isso implica que nos tempos $i \geq 0$ nos quais $[Y(\mathbf{U})]_{i}^{a_{-\infty}^{-1}, 0}=b$, a cadeia $\mathbf{X}(\mathbf{U})^{a_{-\infty}^{-1}, 0}$ é estacionária, pois são tempos nos quais o estado da cadeia é obtido sem precisar do passado (auto-definido, ou definido espontaneamente). Mas isso não é o caso para os tempos $j$ nos quais o valor $(X(\mathbf{U}))_{i}^{a_{-\infty}^{-1}, 0}$ envolve valores da cadeia nos tempos negativos.

O Lema 9.2 nos diz que para todo $n \in \mathbb{Z}$, existe um tempo $\mathbb{Q}$-q.c. finito $\bar{\theta}[n,+\infty]$ tal que

$$
[Y(\mathbf{U})]_{\bar{\theta}[n,+\infty]}^{a_{[\infty}^{-1}, 0}, \ldots,[Y(\mathbf{U})]_{\bar{\theta}[n,+\infty]+\sigma-1}^{a_{-\infty}^{-1}, 0}=b^{\sigma}
$$

e para todo $j \geq \bar{\theta}[n,+\infty]+\sigma$, temos

$$
\bar{h}(j-\bar{t}(j))) \leq j-\bar{\theta}[n,+\infty]+1 .
$$

Isso segue da Definição 9.1 de $\bar{\theta}[n,+\infty]$. Em outras palavras, existe um tempo $T \geq 0$ tal que $\left((X(\mathbf{U}))_{T}^{a_{-\infty}^{-1}, 0}, \ldots,(X(\mathbf{U}))_{T+\sigma-1}^{a_{-\infty}^{-1}, 0}\right)$ é uma sequência não interrompida de $b$ 's (auto-definidos), e para 
todo $j \geq T+\sigma$, os valores da cadeia $\mathbf{X}(\mathbf{U})^{a_{-\infty}^{-1}, 0}$ são obtidos sem utilizar os valores da cadeia antes do tempo T. Em outras palavras

$$
\mathcal{L}\left(U_{T}^{+\infty}\right)=1
$$

Portanto, a partir deste tempo $T$, a cadeia $\left((X(\mathbf{U}))^{a_{-\infty}^{-1}, 0}\right)$ é estacionária.

O argumento acima utiliza o fato que a cadeia $\mathbf{X}(\mathbf{U})^{a_{-\infty}^{-1}, 0}$ é regenerativa, e portanto a construção apresentada na Seção 2.5 é possível a partir de um tempo $T$ no futuro. Este tempo é uma variável aleatória. Ele é distribuído de maneira estacionária, pois a cadeia Y é estacionária. Dessa forma, a cadeia $\mathbf{X}(\mathbf{U})^{a_{-\infty}^{-1}, 0}$ é estacionária após este tempo pelas mesmas razões que na prova do Teorema 2.13. A unicidade da lei estacionária desta cadeia segue do fato que ela é regenerativa.

Na prática, a construtibilidade a partir do tempo $T$ é sem utilidade, porque para ter certeza que este tempo é de fato um tempo de regeneração, precisamos saber os valores da cadeia $\mathbf{U}$ até o infinito no futuro.

Prova do Corolário 5.6. Temos que mostrar que as condições do Corolário 5.6 implicam as condições do Teorema 5.5. Sabemos que o envelope inferior de $\left\{p_{i}(b)\right\}_{i \geq 0}$ (que denotamos $\left\{p_{i}^{\prime}(b)\right\}_{i \geq 0}$ ) verifica

$$
\limsup _{k \rightarrow \infty} \frac{\log \left(\ell^{1}(k)\right)}{\log \left(\frac{1}{1-p_{k}^{\prime}(b)}\right)^{k}}<1
$$

portanto, existe um número inteiro positivo $\bar{k}$ e um número real positivo $\alpha$ tais que para todo $k$ maior que $\bar{k}$

$$
\ell^{1}(k) \leq\left(\frac{1}{1-p_{k}^{\prime}(b)}\right)^{k /(1+\alpha)}
$$

Pela definição de $r^{b}$ (ver (5.4)),

$$
r^{b}(n) \geq \frac{1+\alpha}{\log \frac{1}{1-p_{n}^{\prime}(b)}} \log n
$$

para todo $n$ maior que $\bar{n}$ onde $\bar{n}$ é tal que $r^{b}(\bar{n}) \geq \bar{k}$. Assim

$$
\sum_{n \geq 1}\left(1-p_{n}^{\prime}(b)\right)^{r^{b}(n)} \leq \sum_{n=1}^{\bar{n}-1}\left(1-p_{n}^{\prime}(b)\right)^{r^{b}(n)}+\sum_{n \geq \bar{n}} n^{-1-\alpha}
$$


é finito porque $\alpha$ é positiva. Agora, a condição (5.6) do Corolário 5.6 implica

$$
\sum_{n \geq 1}\left(1-p_{n}^{\prime}(b)\right)^{r^{b}(n)}<\infty
$$

e lembramos a condição (5.7) do Corolário 5.6

$$
\sum_{l \geq 0} \prod_{j=0}^{l-1}\left(1-p_{j}^{\prime}(b)\right)<+\infty
$$

Separamos então dois casos.

1. Quando $r^{b}(l) \leq l$ para $l$ suficientemente grande, então $p_{j}^{\prime}(b) \geq p_{l}^{\prime}(b)$ para $j=0, \ldots r^{b}(l)-1$. Aqui utilizamos o fato que $\left\{p_{n}^{\prime}(b)\right\}_{n \geq 0}$ é decrescente. Portanto,

$$
\sum_{l \geq 0} \prod_{j=0}^{\bar{r}(l)-1}\left(1-p_{j}(b)\right) \leq \sum_{l \geq 0} \prod_{j=0}^{\bar{r}(l)-1}\left(1-p_{j}^{\prime}(b)\right) \leq \sum_{l \geq 0}\left(1-p_{l}^{\prime}(b)\right)^{r^{b}(l)}
$$

e sabemos que este último é finito pelo que dizemos acima utilizando a condição (5.6) do Corolário 5.6. Dessa forma

$$
\sum_{l \geq 0} \prod_{j=0}^{\bar{r}(l)-1}\left(1-p_{j}^{\prime}(b)\right)<+\infty
$$

e as condições do Teorema 5.5 estão satisfeita.

2. Quando $r^{b}(l)>l$ para $l$ suficientemente grande, então

$$
\sum_{l \geq 0} \prod_{j=0}^{\bar{r}(l)-1}\left(1-p_{j}^{\prime}(b)\right) \leq \sum_{l \geq 0} \prod_{j=0}^{l-1}\left(1-p_{j}^{\prime}(b)\right)
$$

e este último é finito pela condição (5.7) do Teorema 5.6. Neste caso também as condições do Teorema 5.5 estão satisfeitas. 


\section{Capítulo 10}

\section{Conclusion}

Nesta tese, abrimos um caminho para estender resultados de existência, unicidade ou ainda simulação perfeita nos casos de família de probabilidades de transição não contínuas. No entanto, de alguma maneira, nossas árvores verificam uma condição de continuidade: considera uma árvore de contextos probabilística $(\tau, p)$, observamos que para todo $x_{-\infty}^{-1} \in A^{-\mathbb{N}}$ tal que $\left|c_{\tau}\left(x_{-\infty}^{-1}\right)\right|<+\infty$, temos que

$$
\beta_{k}\left(x_{-\infty}^{-1}\right)=\sup \left\{\left|p\left(a \mid x_{-k}^{-1} a_{-\infty}^{-k-1}\right)-p\left(a \mid x_{-k}^{-1} b_{-\infty}^{-k-1}\right)\right|: a \in A, a_{-\infty}^{-k-1}, b_{-\infty}^{-k-1}\right\}
$$

converge para 0 quando $k$ diverge. Isso só pode não valer para os ramos infinitos de $\tau$. No entanto, a condição da existência do contexto finito sufixo de $x_{-\infty}^{-1}$ é mais forte do que simplesmente assumir que a convergência acima vale. Portanto, existe uma maneira de estender nossos resultados neste sentido.

Uma outra questão interessante é a questão da simulação perfeita no caso em que não existem símbolos $\epsilon$-regulares. Neste caso, mostramos sob certas condições que vale a existência e unicidade da cadeia, porém, não sabemos construir esta cadeia na medida estacionária.

Enfim, existem outras questões estatísticas, como por exemplo a estimação da árvore a partir de uma realização. As boas propriedades de mistura das nossas cadeias deveriam ajudar neste sentido. 


\section{Referências Bibliográficas}

Bejerano, G. \& Yona, G. (2001). Variations on probabilistic suffix trees: statistical modeling and prediction of protein families. Bioinformatics 17, 23-43. 1

Berbee, H. (1987). Chains with infinite connections: uniqueness and Markov representation. Probab. Theory Related Fields 76(2), 243-253. 8

Bowen, R. (2008). Equilibrium states and the ergodic theory of Anosov diffeomorphisms, vol. 470 of Lecture Notes in Mathematics. Berlin: Springer-Verlag, revised ed. With a preface by David Ruelle, Edited by Jean-René Chazottes. 2

Comets, F., Fernandez, R. \& Ferrari, P. (2002). Processes with long memory: regenerative construction and perfect simulation. Ann. Appl. Prob. 12(3), 921-943. 13, 17, 26

Cover, T. M. \& Thomas, J. A. (2006). Elements of information theory. Hoboken, NJ: WileyInterscience [John Wiley \& Sons], second ed. 43

Doeblin, W. \& Fortet, R. (1937). Sur des chaînes à liaisons complètes. Bull. Soc. Math. France 65, $132-148.8$

Esteves, D. M. (2007). Um esquema regenerativo visível em cadeias de alcance variável não limitada. http://www.teses.usp.br/teses/disponiveis/45/45133/tde-02062007-182457/ . 2, 31

Feller, W. (1968). An introduction to probability theory and its applications. Vol. I. Third edition. New York: John Wiley \& Sons Inc. 70, 89

Fernández, R., Ferrari, P. A. \& Galves, A. (2001). Coupling, Renewal and Perfect Simulations of Chain of Infnite Order. URL http://www.ime.usp.br/ galves/livro/revised2.pdf. $2,6,11$

Fernández, R. \& Galves, A. (2002). Markov approximations of chains of infinite order. Bull. Braz. Math. Soc. (N.S.) 33(3), 295-306. Fifth Brazilian School in Probability (Ubatuba, 2001). 8

Ferrari, P. A., Maass, A., Martínez, S. \& Ney, P. (2000). Cesàro mean distribution of group automata starting from measures with summable decay. Ergodic Theory Dynam. Systems 20(6), 1657-1670. 13

Galves, A., Galves, C., Garcia, N. \& Leonardi, F. (2009). Context tree selection and linguistic rhythm retrieval from written texts. ArXiv: 0902.3619v2, 2009 . 1

Harris, T. E. (1955). On chains of infinite order. Pacific J. Math. 5, 707-724. 8

Keane, M. (1972). Strongly mixing g-measures. Invent. Math. 16, 309-324. 7 
Keane, M. \& Smorodinsky, M. (1977). A class of finitary codes. Israel J. Math. 26(3-4), 352-371. 43

Lalley, S. P. (1986). Regenerative representation for one-dimensional Gibbs states. Ann. Probab. 14(4), 1262-1271. 8, 13

LEONARDi, F. (2006). A generalisation of the pst algorithm: modeling the sparse nature of protein sequences. Bioinformatics 22, 1302-1307. 1

LeOnardi, F. G. (2007). Cadeias estocásticas parcimoniosas com aplicações à classificação e filogenia das sequências de proteínas. http://www.teses.usp.br/teses/disponiveis/95/95131/tde07032007-121126/3 . 1

Lindvall, T. (1992). Lectures on the coupling method. Wiley Series in Probability and Mathematical Statistics: Probability and Mathematical Statistics. New York: John Wiley \& Sons Inc. A Wiley-Interscience Publication. 40

Ornstein, D. S. (1974). Ergodic theory, randomness, and dynamical systems. New Haven, Conn.: Yale University Press. James K. Whittemore Lectures in Mathematics given at Yale University, Yale Mathematical Monographs, No. 5. 43

Propp, J. G. \& Wilson, D. B. (1996). Exact sampling with coupled Markov chains and applications to statistical mechanics. In: Proceedings of the Seventh International Conference on Random Structures and Algorithms (Atlanta, GA, 1995), vol. 9.13

Rissanen, J. (1983). A universal data compression system. IEEE Trans. Inform. Theory 29(5), 656-664. 1

Stenflo, Ö. (2003). Uniqueness in g-measures. Nonlinearity 16(2), 403-410. 8

Thorisson, H. (2000). Coupling, stationarity, and regeneration. Probability and its Applications (New York). New York: Springer-Verlag. 37, 40 